\title{
Power series expansions of modular forms and $p$-adic interpolation of the square roots of Rankin-Selberg special values
}

\author{
Andrea Mori \\ Dipartimento di Matematica \\ Università di Torino
}

\begin{abstract}
Let $f$ be a newform of even weight $2 \kappa$ for $D^{\times}$, where $D$ is a possibly split indefinite quaternion algebra over $\mathbb{Q}$. Let $K$ be a quadratic imaginary field splitting $D$ and $p$ an odd prime split in $K$. We extend our theory of $p$-adic measures attached to the power series expansions of $f$ around the Galois orbit of the CM point corresponding to an embedding $K \hookrightarrow D$ to forms with any nebentypus and to $p$ dividing the level of $f$. For the latter we restrict our considerations to CM points corresponding to test objects endowed with an arithmetic $p$-level structure. Also, we restrict these $p$-adic measures to $\mathbb{Z}_{p}^{\times}$and compute the corresponding Euler factor in the formula for the $p$-adic interpolation of the "square roots"of the Rankin-Selberg special values $L\left(\pi_{K} \otimes \xi_{r}, \frac{1}{2}\right)$ where $\pi_{K}$ is the base change to $K$ of the automorphic representation of $\mathrm{GL}_{2}$ associated, up to Jacquet-Langland correspondence, to $f$ and $\xi_{r}$ is a compatible family of grössencharacters of $K$ with infinite type $\xi_{r, \infty}(z)=(z / \bar{z})^{\kappa+r}$.
\end{abstract}

Modular forms; automorphic $L$-functions.

Mathematics Subject Classification 2010: 11F67

\section{Introduction}

Let $f$ be a holomorphic modular newform of weight $2 \kappa$, level $N$ and nebentypus $\varepsilon$ for $D^{\times}$, where $D$ is the possibly split indefinite quaternion algebra of discriminant $\Delta$ with $(\Delta, N)=1$. Let $K$ be a quadratic imaginary field splitting $D$ in which the odd prime $p$ splits, $p \mathcal{O}_{K}=\mathfrak{p} \bar{p}$. In [26] we used the power series expansions of $f$ in terms of the Serre-Tate parameter around the Galois conjugates of the CM point $\tau \in \mathfrak{H}$ associated to an embedding $\rho: K \hookrightarrow D$ to construct, under the hypotheses that $f$ has trivial nebentypus and $p$ is prime to the level $N$, a $p$-adic measure on $\mathbb{Z}_{p}$ whose $r$-th moment squared is strictly related to the special Rankin-Selberg value $L\left(\pi_{K} \otimes \xi_{r}, \frac{1}{2}\right)$. Here $\pi_{K}$ is the base change to $K$ of the automorphic representation $\pi$ of $\mathrm{GL}_{2}$ defined by $f$ up to Jacquet-Langlands correspondence and $\xi_{r}$ is a certain family of grössencharacters of $K_{\mathbb{A}}^{\times}$. 
The main goal of this paper is to compute the restriction of this measure to $\mathbb{Z}_{p}^{\times}$or, in other words, to identify the correct interpolating Euler factor. At the same time, we extend the theory to forms of any nebentypus and to primes dividing the level. While the former extension is formal, the latter requires some adjustement. Since modular and Shimura curves are not smooth mod $p$ for $p$ dividing the level we consider only $p$-ordinary split arithmetic test objects, essentially CM points corresponding to elliptic curves, or QM abelian surfaces, defined over the integers in a finite extension of $K_{\mathfrak{p}}$ endowed with an arithmetic level structure at $p$ (see definition 1.2). On these points the effects of the classical and $p$-adic operators $U$ and $V$ coincide. This allows to connect the effect of restricting the measure to $\mathbb{Z}_{p}^{\times}$to the variation under the classical $V$ operator of the toric period $J_{r}(f, \xi, \tau) \stackrel{p}{=} \int_{K_{\mathrm{A}}^{\times} / K^{\times} \mathbb{R}^{\times}} \phi_{r}\left(t d_{\infty}^{\tau}\right) \xi(t) d t$, where $\phi_{r}$ is the adelic lift of $\delta_{2 \kappa}^{(r)} f\left(\delta_{2 \kappa}^{(r)}\right.$ is the $r$-th iterate, in the automorphic sense, of the basic Maass operator $), d_{\infty}^{\tau} \in \mathrm{SL}_{2}(\mathbb{R})$ is the standard parabolic matrix such that $d_{\infty}^{\tau} \cdot i=\tau$ and $\xi$ is a grössencharacter of $K_{\mathbb{A}}^{\times}$of weight $(-\kappa-r, \kappa+r)$ that has a prescribed reduction over $\widehat{\mathcal{O}}_{K, c}^{\times}$, where $c$ is the conductor relative to $N$ of the embedding $\rho$. The toric integrals $J_{r}(f, \xi, \tau)$ provide the link between the expansions of $f$ and the special Rankin-Selberg values. On one side, they can be computed in terms of a coupling defined on the $p$-adic space of $p$-adic forms for $\mathrm{GL}_{1}(K)$, which happens to be bounded on the closed space of avatars of forms of bounded level. On the other hand, their squares are linked to the special Rankin-Selberg $L$-values, as originally proved by Waldspurger, [30]. In doing so we follow [10], where each $J_{r}(f, \xi, \tau)^{2}$ is a special case of a generalized Fourier coefficient for an automorphic form on $R_{K / \mathbb{Q}} \mathbb{G}_{m}^{2}$. Building on results of Shimizu [28] and refining the techniques of Waldspurger, Harris and Kudla use the seesaw identity associated with the theta correspondence between the similitude groups $\mathrm{GL}_{2}$ and $\operatorname{GO}(D)$ and the splitting $D=K \oplus K^{\perp}$ to express the generalized Fourier coefficients of a theta lift $\theta_{\varphi}(F)$, where $F \in \pi$ and $\varphi$ is a split primitive SchwartzBruhat function on $D_{\mathbb{A}}$, as Rankin-Selberg Euler products. The theory can be made more explicit using the local computations of Watson [31] (see also [27]) that allow to pinpoint a precise Schwartz-Bruhat function outside a finite set of "bad" primes. In section 3.3 we deal with some of the local cases that were not in the scope of [31] and [27], in particular when the level is not square-free or when the local component of the central character is not trivial.

We now state the main result of the paper.

Theorem 0.1. Let $K \subset D$ be an embedding of conductor $c$ relative to $N$ with fixed point $\tau \in \mathfrak{H}$ corresponding to a p-ordinary split arithmetic test object, $y=\operatorname{Im}(\tau)$. Assume $(p, c)=1$ and $\mathcal{O}_{K, c}^{\times}=\{ \pm 1\}$. For $r \geq 0$ let $\chi_{r}$ be $a$ grössencharacter of $K_{\mathbb{A}}^{\times}$of infinity type $\chi_{r, \infty}(z)=z^{2(\kappa+r)}$ compatible with the central character $\varepsilon$ whose $p$-adic avatar is the $r$-th moment of a measure on $\mathbb{Z}_{p}$ with values in the p-adic space $\mathfrak{F}\left(c^{*}, F\right)$ for a sufficiently large local extension $K_{\mathfrak{p}} \subset F$. Let $\xi_{r}=\chi_{r}\left\|N_{K / \mathbb{Q}}\right\|^{-\kappa-r}$. 
Then there exists a p-adic measure $\mu$ on $\mathbf{C}_{c}^{\sharp} \times \mathbb{Z}_{p}^{\times}$such that

$$
\begin{array}{r}
\frac{1}{\Omega_{p}^{4(\kappa+r)}}\left(\int_{\mathbf{C}_{c}^{\sharp} \times \mathbb{Z}_{p}^{\times}} \psi(s) t^{r} d \mu(s, t)\right)^{2}=\frac{v}{\left(\operatorname{vol}\left(\hat{\mathcal{O}}_{K, c}^{\times}\right) h_{c}^{\sharp}\right)^{2}} \frac{\pi^{2 \kappa+r-3}}{\left(y \Omega_{\infty}^{2}\right)^{2(\kappa+r)}} \times \\
E_{\mathfrak{p}}\left(\psi \xi_{r}\right)^{2} \Lambda\left(\varphi_{r}^{\sharp}, \psi \xi_{r}\right) L\left(\pi_{K} \otimes \psi \xi_{r}, \frac{1}{2}\right) L\left(\eta_{K}, 1\right)^{-1}
\end{array}
$$

where

1. $v$ is a normalizing ratio of Petersson norms, $\Omega_{\infty}$ and $\Omega_{p}$ are archimedean and $p$-adic periods respectively;

2. $E_{\mathfrak{p}}\left(\psi \xi_{r}\right)=\left(1-a_{p}\left(\psi \xi_{r}\right)_{\mathfrak{p}}\left(\varpi_{\mathfrak{p}}\right) p^{-2 \kappa}+\varepsilon(p)\left(\psi \xi_{r}\right)_{\mathfrak{p}}\left(\varpi_{\mathfrak{p}}\right)^{2} p^{-2 \kappa-1}\right)$, where $a_{p}$ is the $p$-th Hecke eigenvalue of $f$ and $\varpi_{\mathfrak{p}}$ is a local uniformizer in $K_{\mathfrak{p}}$ well defined up to a unit in the conductor of $\xi_{r, \mathfrak{p}}$;

3. the factor $\Lambda\left(\varphi_{r}, \psi \xi_{r}\right) \in \overline{\mathbb{Q}}$ is a product of local terms almost all of them equal to 1: it depends on an explicit Schwartz-Bruhat function $\varphi_{r}^{\sharp}=$ $\varphi_{\infty}^{\sharp(r, r)} \otimes \bigotimes_{\ell<\infty} \phi_{\ell}^{\prime}$ which varies with $r$ only in the archimedean component.

The compatibility of $\chi_{r}$, hence $\xi_{r}$, with the central character, which had been already hinted in the introductory remarks, is in the sense of proposition 3.2: in particular it is required that $\xi_{r \mid \mathbb{A} \times}=\varepsilon^{-1}$. One should notice that the compatibility condition depends strongly on $\rho$, so, in general, changing the embedding results in a $p$-adic interpolation of an entirely different set of twists. A standard way to produce a family of grössencharacters $\xi_{r}$ as requested is outlined in remark 2.18.

The link with the couplings discussed in section 2.3, which has already been alluded to above, is also a strong clue for the non-vanishing of possibly many of the special values $L\left(\pi_{K} \otimes \psi \xi_{r}, \frac{1}{2}\right)$ as $\xi_{r}$ is kept fixed and $\psi$ varies in $\widehat{\mathbf{C}}_{c}^{\sharp}$. In order to be able to give a quantitative version of this expectation, one needs a tight control of the values of $\delta^{r}(f)$ at the Galois orbit of the relevant CM point.

The idea that the coefficients of the power series expansions at CM points of a modular form (that had been introduced in [25] with a different goal) could be used to interpolate $p$-adically special values $L\left(\pi_{K} \otimes \xi, \frac{1}{2}\right)$ arose a long time ago in private conversations with $M$. Harris and was mentioned for the first time in [12]. Not long after [26] came out in print and already a good deal of the material for the present paper had been worked out we became aware of the works of M. Bertolini, H. Darmon and K, Prasanna [1] and M. Brakočević [2] where such $p$-adic interpolations had been obtained. The main goal of [1] is far beyond the mere construction of a $p$-adic $L$-function and the authors work under less inclusive hypotheses and the techniques used appear substantially different. On the other hand the main goal of [2] is to construct a "cuspidal" measure in the spirit of Katz's classical paper [20], a goal that turned out to be tightly related to our looking for arithmetic applications of the power series expansions other than a mere analogue of the classical $q$-expansion principle (valid only 
in the split case). Although the techniques employed are not exactly the same and the main result is somewhat different, there is a good overlap between the ideas employed in Brakočević's paper and in here. While Brakočević's main result is more invariant and better fitted for applications as he constructs a unique measure on the profinite group $\mathrm{Cl}_{K}^{-}\left(N_{\mathrm{ns}} p^{\infty}\right)$ (his notation, where $N_{n s}$ is the non-split part of the level $N$ ) that interpolates a large deal of twists, our approach makes an explicit use of the power series expansions at points in the non-split Shimura curves. Since the use of expansions in the anisotropic case seems interesting per se and to the best of our knowledge unprecedented, we decided, even after such long time, to complete the present work in its final form.

\section{Notations and Conventions.}

Prime numbers are denoted $\ell$ (possibly $\ell=\infty$ with the convention that $\mathbb{Q}_{\infty}=\mathbb{R}$, depending on context). When a finite prime number is to be supposed fixed we denote it $p$ and we fix once for all embeddings $i_{\infty}: \overline{\mathbb{Q}} \rightarrow \mathbb{C}$ and $i_{p}: \overline{\mathbb{Q}} \rightarrow \mathbb{C}_{p}$. If $v$ is a finite place in the number field $L$ with ring of integers $\mathcal{O}_{L}$, we denote $\mathcal{O}_{(v)}$ the ring of $v$-integers in $L$, while $L_{v}, \mathcal{O}_{v}$ and $k_{v}$ are the $v$-adic completion of $L$, of $\mathcal{O}_{L}$ and the residue field respectively. A quadratic imaginary field is always denoted $K$ and if $c$ a positive integer, $\mathcal{O}_{K, c}=\mathbb{Z}+c \mathcal{O}_{K}$ is its order of conductor c. If $\mathfrak{n} \subset \mathcal{O}_{K}$ is an ideal denote $\mathbf{C}_{\mathfrak{n}}=\mathcal{I}_{\mathfrak{n}} / P_{\mathfrak{n}}=K_{\mathbb{A}}^{\times} / K^{\times} \mathbb{C}^{\times} U_{\mathfrak{n}}$ the group of $\mathfrak{n}$-ideal classes of $K$ and $h_{\mathfrak{n}}=\left|\mathbf{C}_{\mathfrak{n}}\right|$. Also, let $\mathbf{C}_{c}^{\sharp}=\mathbf{C}_{c \mathcal{O}_{K}}^{\sharp}=K_{\mathbb{A}}^{\times} / K^{\times} \mathbb{C}^{\times} \widehat{\mathcal{O}}_{K, c}^{\times}$ and $h_{c}^{\sharp}=\left|\mathbf{C}_{c}^{\sharp}\right|$.

Fix an additive character $\psi$ of $\mathbb{A} / \mathbb{Q}(\mathbb{A}=\mathbb{Q} \mathbb{R} \widehat{\mathbb{Z}}$ is the ring of rational adeles $)$ asking that $\psi_{\infty}(x)=e^{2 \pi i x}$ and $\psi_{\ell}$ is trivial on $\mathbb{Z}_{\ell}$ with $\psi_{\ell}(x)=e^{2 \pi i x}$ for $x \in \mathbb{Z}\left[\ell^{-1}\right]$ and finite $\ell$. This determines the Haar measure $d x=\prod_{\ell \leq \infty} d x_{\ell}$ on $\mathbb{A}$, where the local Haar measures $d x_{\ell}$ are normalized so that the $\bar{\psi}_{\ell}$-Fourier transform is autodual. On $\mathbb{A}^{\times}$we fix the multiplicative Haar measure $d^{\times} x=$ $\prod_{\ell \leq \infty} d^{\times} x_{\ell}$ where $d^{\times} x_{\ell}=\zeta_{\ell}(1)|x|_{\ell}^{-1} d x_{\ell}$, so that $\operatorname{vol}\left(\mathbb{Z}_{\ell}^{\times}\right)=1$ for finite $\ell$. For a quaternion algebra $D$ the Haar measure $d x=\prod_{\ell \leq \infty} d x_{\ell}$ on $D_{\mathbb{A}}$ is fixed requiring that the local measures $d x_{\ell}$ are normalized so that the Fourier transform with respect to the norm form is autodual. On $D(\mathbb{A})^{\times}$we fix the multiplicative measure $d^{\times} x=\prod_{\ell} d^{\times} x_{\ell}$ where $d^{\times} x_{\ell}=\zeta_{\ell}(1)|x|_{\ell}^{-1} d x_{\ell}$ on $\left(D \otimes \mathbb{Q}_{\ell}\right)^{\times}$, so that $\operatorname{vol}\left(\mathrm{GL}_{2}\left(\mathbb{Q}_{\ell}\right)\right)=\zeta_{\ell}(2)^{-1}$ when $D=\mathrm{GL}_{2}$.

Given a quadratic space $(V,\langle\rangle$,$) of dimension d$ over $\mathbb{Q}$ denote $\mathcal{S}_{\mathbb{A}}(V)=$ $\bigotimes_{\ell<\infty} \mathcal{S}_{\ell}$ the adelic Schwartz-Bruhat space: for $\ell$ finite, $\mathcal{S}_{\ell}$ is the space of locally constant functions on $V \otimes \mathbb{Q}_{\ell}$ with compact support and $\mathcal{S}_{\infty}$ is the space of Schwartz functions on $V \otimes \mathbb{R}$ which are finite under the natural action of a (fixed) maximal compact subgroup of the similitude group $\mathrm{GO}(V)$. The Weil representation $r_{\psi}$ is the representation of $\mathrm{SL}_{2}(\mathbb{A})$ on $\mathcal{S}_{\mathbb{A}}(V)$ which is explicitly described locally at $\ell \leq \infty$ by 


$$
\begin{aligned}
r_{\psi}\left(\begin{array}{cc}
1 & b \\
0 & 1
\end{array}\right) \varphi(x) & =\psi_{\ell}\left(\frac{1}{2}\langle b x, x\rangle\right) \varphi(x), \\
r_{\psi}\left(\begin{array}{cc}
a & 0 \\
0 & a^{-1}
\end{array}\right) \varphi(x) & =\chi_{V}(a)|a|_{\ell}^{d / 2} \varphi(a x) \\
r_{\psi}\left(\begin{array}{cc}
0 & 1 \\
-1 & 0
\end{array}\right) \varphi(x) & =\gamma_{V} \hat{\varphi}(x)
\end{aligned}
$$

where $\gamma_{V}$ is an eighth root of 1 and $\chi_{V}$ is a quadratic character that are computed in our cases of interest in [16] (see also [27, §3.4]), while $\hat{\varphi}(x)=$ $\int_{V \otimes \mathbb{Q}_{\ell}} \varphi(y) \psi_{\ell}(\langle x, y\rangle) d y$ is the Fourier transform computed with respect to a $\langle$,$\rangle -self dual Haar measure on V \otimes \mathbb{Q}_{\ell}$.

The group $\mathrm{GL}_{2}^{+}(\mathbb{R})$ acts on the upper half-plane $\mathfrak{H}=\{\tau=x+y i \in \mathbb{C} \mid y>0\}$ by linear fractional transformations: $\left(\begin{array}{ll}a & b \\ c & d\end{array}\right) \cdot \tau=\frac{a \tau+b}{c \tau+d}$. The automorphy factor is $j(g, \tau)=c \tau+d$. Given $\tau=x+y i \in \mathfrak{H}$ let $d_{\infty}^{\tau}=\sqrt{y}\left(\begin{array}{ll}1 & x / y \\ 0 & 1 / y\end{array}\right) \in \mathrm{SL}_{2}(\mathbb{R})$ the upper triangular matrix such that $d_{\infty}^{\tau} \cdot i=\tau$. The space of holomorphic modular forms with respect to the Fuchsian groups of the first kind $\Gamma_{*}^{\Delta}(N), * \in\{0,1\}$, whose definition is recalled in section 1.1, will be denoted $M_{*, k}^{\Delta}(N)$. The bigger spaces of $\mathcal{C}^{\infty}$-modular forms for whom only analiticity is required, mantaining the growth conditions at the cusps, will be denoted $M_{*, k, \infty}^{\Delta}(N)$.

\section{Background}

\subsection{Modular and Shimura curves.}

Let $D$ be an indefinite quaternion algebra over $\mathbb{Q}$ with reduced norm $\nu$ and reduced trace tr. For every rational place $\ell$ let $D_{\ell}=D \otimes \mathbb{Q}_{\ell}$. Fix an isomorphism $\Phi_{\infty}: D_{\infty} \stackrel{\sim}{\longrightarrow} \mathrm{M}_{2}(\mathbb{R})$ which will be usually left implicit. Let $\Sigma_{D}$ be the finite and even set of places at which $D$ is ramified and let $\Delta=\Delta_{D}=\prod_{\ell \in \Sigma_{D}} \ell$ be the discriminant of $D$. If $\mathcal{R} \subset D$ is an order and $\ell<\infty$ let $\mathcal{R}_{\ell}=\mathcal{R} \otimes \mathbb{Z}_{\ell}$. Fix:

- A maximal order $\mathcal{R} \subset D$, with isomorphisms $\Phi_{\ell}: D_{\ell} \stackrel{\sim}{\longrightarrow} \mathrm{M}_{2}\left(\mathbb{Q}_{\ell}\right)$ for all finite $\ell \notin \Sigma_{D}$ such that $\Phi_{\ell}\left(\mathcal{R}_{\ell}\right)=\mathrm{M}_{2}\left(\mathbb{Z}_{\ell}\right)$. For every $N$ such that $(N, \Delta)=1$ let $\mathcal{R}_{N} \subset \mathcal{R}$ be the Eichler order given by the local conditions

$$
\Phi_{\ell}\left(\mathcal{R}_{N, \ell}\right)=\left(\left\{\left(\begin{array}{ll}
a & b \\
c & d
\end{array}\right) \in \mathrm{M}_{2}\left(\mathbb{Z}_{\ell}\right) \text { such that } c \equiv 0 \bmod N\right\}\right)
$$

for $\ell \notin \Sigma_{D}$, and $\mathcal{R}_{N, \ell}$ is the unique maximal order in $D_{\ell}$ for $\ell \in \Sigma_{D}$. It is equipped with homomorphisms ( $\ell$-orientations) or $_{\ell}^{1}$, or $_{\ell}^{2}: \mathcal{R}_{N} \otimes \mathbb{F}_{\ell} \rightarrow \mathbb{F}_{\ell^{2}}$ for $\ell \mid \Delta$, and or $_{\ell}^{1}$, or $_{\ell}^{2}: \mathcal{R}_{N} \otimes \mathbb{Z} / \ell^{s} \mathbb{Z} \rightarrow\left(\mathbb{Z} / \ell^{s} \mathbb{Z}\right)^{2}$ for $\ell^{s} \| N$. If $D=\mathrm{M}_{2}(\mathbb{Q})$ we take $\mathcal{R}=\mathrm{M}_{2}(\mathbb{Z})$.

- A positive involution $d \mapsto d^{\dagger}=t^{-1} \bar{d} t$ with $t \in \mathcal{R}$ and $t^{2}=-\Delta$. Thus $\mathcal{R}^{\dagger}=$ $\mathcal{R}$ and the skew-symmetric bilinear form $B_{t}(a, b)=\operatorname{tr}(a \bar{b} t)=\operatorname{tr}\left(a t b^{\dagger}\right)$ is non-degenerate and $\mathbb{Z}$-valued on $\mathcal{R} \times \mathcal{R}$. 
Consider the groups

$$
\begin{gathered}
\Gamma_{0}^{\Delta}(N)=\mathcal{R}_{N}^{1}=\left\{\gamma \in \mathcal{R}_{N} \text { such that } \nu(\gamma)=1\right\} \quad \text { and } \\
\Gamma_{1}^{\Delta}(N)=\left\{\gamma \in \mathcal{R}_{N}^{1} \text { such that } \operatorname{or}_{\ell}^{\epsilon}(\bar{\gamma})=1 \text { for } \ell \mid N, \epsilon=1,2\right\} .
\end{gathered}
$$

When $\Delta=1, \Gamma_{0}^{1}(N)$ and $\Gamma_{1}^{1}(N)$ are the classical congruence subgroups $\Gamma_{0}(N)$ and $\Gamma_{1}(N)$ respectively. The groups $\Gamma_{*}^{\Delta}(N)$ are, via $\Phi_{\infty}$, discrete subgroups of $\mathrm{SL}_{2}(\mathbb{R})$ acting on $\mathfrak{H}$. When $\Delta>1$ the quotient $X_{*}^{\Delta}(N)=\Gamma_{*}^{\Delta}(N) \backslash \mathfrak{H}$ is a compact Riemann surface. When $\Delta=1$ let $X_{*}^{1}(N)$ be the standard cuspidal compactification of $Y_{*}(N)=\Gamma_{*}(N) \backslash \mathfrak{H}$. Each of these curves $X_{*}^{\Delta}(N)$ is the set of complex points of a proper scheme $\mathcal{X}_{*}^{\Delta}(N)$ smooth over $\mathbb{Z}[1 / N \Delta]$ which is the solution, for $N$ not too small and $*=1$, of a representable moduli problem defined over $\mathbb{Z}[1 / \Delta]$.

When $\Delta=1, N>3$ the scheme $\mathcal{X}_{1}^{1}(N)$ is the compactified moduli space for the functor $F_{1}^{1}(N)$ : Schemes $\rightarrow$ Sets defined by

$$
F_{1}^{1}(N)(S)=\left\{\begin{array}{l}
\text { Isomorphism classes of elliptic curves } \\
E_{/ S} \text { with a } \Gamma_{1}(N) \text {-structure. }
\end{array}\right\}
$$

A $\Gamma_{1}(N)$-structure on an elliptic curve $E_{/ S}$ is a section $P: S \rightarrow E$ such that the effective Cartier divisor $\sum_{d \in \mathbb{Z} / N \mathbb{Z}} d P$ is a subgroup scheme of $E$. Denote $\pi_{N}: \mathcal{E}_{N} \rightarrow \mathcal{X}_{1}^{1}(N)$ the corresponding universal (generalized) elliptic curve. For $S=\operatorname{Spec}(\mathbb{C})$ the fiber corresponding to $z \in \mathfrak{H}$ is the torus $E_{z}=\mathbb{C} / \mathbb{Z} \oplus \mathbb{Z} z$ with point $P=1 / N \bmod \mathbb{Z}$. When $\Delta>1$ and $N>3$, the scheme $\mathcal{X}_{1}^{\Delta}(N)$ represents the functor $F_{1}^{\Delta}(N): \mathbb{Z}[1 / \Delta]$-Schemes $\rightarrow$ Sets defined by

$$
F_{1}^{\Delta}(N)(S)=\left\{\begin{array}{l}
\text { Isomorphism classes of compatibly principally po- } \\
\text { larized abelian surfaces } A_{/ S} \text { with a ring embedding } \\
\iota: \mathcal{R} \hookrightarrow \operatorname{End}_{S}(A) \text { and a } \Gamma_{1}(N) \text {-structure }
\end{array}\right\} .
$$

An abelian surface $A_{/ S}$ with a ring embedding $\iota: \mathcal{R} \hookrightarrow \operatorname{End}_{S}(A)$ is said to have quaternionic multiplications and is called a QM-abelian surface (or false elliptic curve). A principal polarization on a QM-abelian surface is compatible with the embedding $\iota: \mathcal{R} \subset \operatorname{End}_{S}(A)$ if the involution $d \mapsto d^{\dagger}$ is the Rosati involution. Given our choice of involution, there exists a unique compatible principal polarization on a given QM-abelian surface $(A, \iota)$. A $\Gamma_{1}(N)$-structure on a QMabelian surface $A_{/ S}$ is the datum of a section $P: S \rightarrow A$ such that the effective Cartier divisor $\sum_{d \in \mathbb{Z} / N \mathbb{Z}} d P$ is a rank $N$ subgroup of $\operatorname{ker}\left(\left(\begin{array}{ll}1 & 0 \\ 0 & 0\end{array}\right): A[N] \rightarrow A[N]\right)$ where $\left(\begin{array}{ll}1 & 0 \\ 0 & 0\end{array}\right) \in \mathrm{M}_{2}(\mathbb{Z} / N \mathbb{Z}) \simeq \mathcal{R} \otimes(\mathbb{Z} / N \mathbb{Z})$ acts on $A[N]$ via endomorphisms. Denote $\pi_{\Delta, N}: \mathcal{A}_{\Delta, N} \longrightarrow \mathcal{X}_{1}^{\Delta}(N)$ the corresponding universal QM-abelian surface. For $S=\operatorname{Spec}(\mathbb{C})$ the fiber corresponding to $z \in \mathfrak{H}$ is the torus $A_{z}=D_{\infty}^{z} / \mathcal{R}$ where $D_{\infty}^{z}$ denotes the real vector space $D_{\infty}$ endowed with the $\mathbb{C}$-structure defined by the identification $\mathbb{C}^{2}=\Phi_{\infty}\left(D_{\infty}\right)\left(\begin{array}{c}z \\ 1\end{array}\right)$, i.e. $A_{z}=\mathbb{C}^{2} / \Phi_{\infty}(\mathcal{R})\left(\begin{array}{l}z \\ 1\end{array}\right)$. The skew-symmetric form $\left\langle\Phi_{\infty}(a)\left(\begin{array}{c}z \\ 1\end{array}\right), \Phi_{\infty}(b)\left(\begin{array}{c}z \\ 1\end{array}\right)\right\rangle=B_{t}(a, b)$ extended to $\mathbb{C}^{2}$ by $\mathbb{R}$-linearity is the unique Riemann form on $A_{z}$ with Rosati involution $d \mapsto d^{\dagger}$.

The schemes $\mathcal{X}_{0}^{\Delta}(N)$ are constructed from the schemes $\mathcal{X}_{1}^{\Delta}(N)$ by taking quotients by the action of the quotient group $\Gamma_{1}^{\Delta} / \Gamma_{0}^{\Delta} \simeq(\mathbb{Z} / N \mathbb{Z})^{\times}$which can 
be described modularly as $P \mapsto d P$ (diamond operators). They are the coarse moduli schemes attached to the functor $F_{0}^{\Delta}(N)$ obtained replacing the section $P$ in the moduli problem datum by a closed locally free cyclic subgroup scheme of rank $N$ which in the case $\Delta>1$ needs to be a subgroup scheme of $\operatorname{ker}\left(\left(\begin{array}{ll}1 & 0 \\ 0 & 0\end{array}\right): A[N] \rightarrow A[N]\right)$.

When $\ell \mid N$ the structure of the non-smooth fibers $\mathcal{X}_{*}^{\Delta}(N) \otimes \mathbb{F}_{\ell}$ are very similar and have been determined in [22] when $\Delta=1$ and in $[4,5]$ adapting the techniques of Katz and Mazur to the case of QM-abelian surfaces. Write $N=N^{\prime} \ell^{s}$ with $\left(N^{\prime}, \ell\right)=1$. Then

$$
\mathcal{X}_{1}^{\Delta}\left(N^{\prime} \ell^{s}\right) \otimes \mathbb{F}_{\ell}=\coprod_{r=0}^{s} \mathcal{Z}_{1}^{\Delta}(r)
$$

where the component $\mathcal{Z}_{1}^{\Delta}(r)$ has multiplicity $\phi\left(\ell^{s-r}\right)$. The reduced scheme $\mathcal{Z}_{1}^{\Delta}(r)^{\text {red }}$ is the Igusa curve $\mathcal{X}_{1}^{\Delta}\left(N^{\prime}, \operatorname{Ig}\left[\ell^{r}\right]\right) / \mathbb{F}_{\ell}$ (cuspidally compactified when $\Delta=1)$, i.e. the smooth $\mathbb{F}_{\ell}$-scheme that represents the functor $\operatorname{Ig}\left(F_{1}^{\Delta}\left(N^{\prime}\right), \ell^{r}\right)$

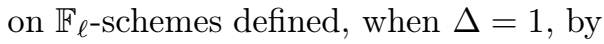

$$
\operatorname{Ig}\left(F_{1}\left(N^{\prime}\right), \ell^{r}\right)(S)=\left\{\begin{array}{l}
\text { Isomorphism classes of elliptic curves } E_{/ S} \text { with } \\
\text { a } \Gamma_{1}\left(N^{\prime}\right) \text {-structure and a choice of a generator } \\
\text { of } \operatorname{ker}\left(\operatorname{Ver}^{r}: E^{\left(\ell^{r}\right)} \rightarrow E\right) .
\end{array}\right\} .
$$

and similarly in the $\Delta>1$ case, where an Igusa $\ell^{r}$-structure on a QM-abelian surface $A_{/ S}$ is a choice of generator of $\left(\begin{array}{ll}1 & 0 \\ 0 & 0\end{array}\right) \operatorname{ker}\left(\operatorname{Ver}^{r}: A^{\left(\ell^{r}\right)} \rightarrow A\right)$. Moreover, in either case all the components meet transversally at each supersingular point. The description of $\mathcal{X}_{0}^{\Delta}\left(N^{\prime} \ell^{s}\right) \otimes \mathbb{F}_{\ell}$ is completely analogous, [22, §13.5.6].

Remark 1.1. Let $\mathcal{X}_{\mathrm{ar}}^{\Delta}(N) \hookrightarrow \mathcal{X}_{1}^{\Delta}(N)$ be the smooth open subscheme obtained by discarding the non-reduced components at the primes $\ell \mid N$. The above modular description in terms of Igusa curves entails, by Cartier duality, that $\mathcal{X}_{\mathrm{ar}}^{1}(N)$ represents the functor on schemes

$$
F_{\text {ar }}^{1}(N)(S)=\left\{\begin{array}{l}
\text { Isomorphism classes of elliptic curves } E_{/ S} \text { with } \\
\text { an embedding } \mu_{N} \hookrightarrow E \text { of } S \text {-group schemes }
\end{array}\right\}
$$

and, when $\Delta>1, \mathcal{X}_{\text {ar }}^{\Delta}(N)$ represents the functor on $\mathbb{Z}[1 / \Delta]$-schemes

$$
F_{\text {ar }}^{\Delta}(N)(S)=\left\{\begin{array}{l}
\text { Isomorphism classes of QM-abelian sur- } \\
\text { faces } A_{/ S} \text { with an } \mathcal{R} \text {-equivariant embed- } \\
\text { ding } \mu_{N} \times \mu_{N} \hookrightarrow A \text { of } S \text {-group schemes }
\end{array}\right\}
$$

These structures are called $N$-level arithmetic structures.

\subsection{CM points.}

Let $\mathbb{Q} \subseteq E \subset F$ be fields with $[F: E]=2, F=E(\alpha)$ with $\alpha^{2}=a \in E$, and $F$ splitting $D$. An embedding $\rho: F \hookrightarrow D \otimes_{\mathbb{Q}} E$ endows $D \otimes_{\mathbb{Q}} E$ with a $F$-vector 
space structure: scalar multiplication by $\lambda \in F$ is left multiplication by $\rho(\lambda)$. If $\operatorname{Gal}(F / E)=\{1, \sigma\}$ let $\rho^{\sigma}=\rho \circ \sigma$. By the Skolem-Noether theorem there exists $u \in\left(D \otimes_{\mathbb{Q}} E\right)^{\times}$, well defined up to a $F^{\times}$-multiple, such that $u \rho(\lambda)=\rho^{\sigma}(\lambda) u$ for all $\lambda \in F$, and $u^{2} \in E$. With a slight abuse of notation a splitting $D \otimes_{\mathbb{Q}} E=F \oplus$ $F u$ is obtained, more intrinsically seen either as the eigenspace decomposition under right multiplication by $\rho\left(F^{\times}\right)$or an orthogonal decomposition under the norm form. The projection onto $F$ with kernel $F^{\perp}=F u$ is the idempotent $e_{\rho}$ image of $\frac{1}{2}\left(1 \otimes 1+\frac{1}{a} \alpha \otimes \alpha\right)$ under

$$
F \otimes_{E} F \stackrel{\rho \otimes \rho}{\longrightarrow}\left(D \otimes_{\mathbb{Q}} E\right) \otimes_{E}\left(D^{\mathrm{op}} \otimes_{\mathbb{Q}} E\right) \simeq \operatorname{End}_{E}\left(D \otimes_{\mathbb{Q}} E\right) .
$$

An involution $d \mapsto d^{\imath}$ in $D$ extends by linearity to $D \otimes_{\mathbb{Q}} E$. If $\rho^{\imath}$ is the embedding $\rho^{\imath}(\lambda)=\rho(\lambda)^{\imath}$, then $e_{\rho^{\imath}}=\left(e_{\rho}\right)^{\imath}$ and in particular $\left(e_{\rho}\right)^{\imath}=e_{\rho}$ if and only if $\rho(F)^{\imath}=\rho(F)$ pointwise.

Let $E=\mathbb{Q}$ from now on. The positivity of the involution $d \mapsto d^{\dagger}$ implies the existence of a traceless element $\delta \in D$ such that $\delta=\delta^{\dagger}$, so that $F=\mathbb{Q}(\delta) \subset D$ is a real quadratic subfield pointwise fixed by the involution and the corresponding idempotent $e \in D \otimes_{\mathbb{Q}} F$ satisfies $e^{\dagger}=e$.

Now let $F=K$, a quadratic imaginary field. In each $\operatorname{Gal}(K / \mathbb{Q})$-orbit in $\operatorname{Hom}(K, D)$ there is exactly one embedding which is normalized in the sense of $[29,(4.4 .5)]$. The normalized embeddings correspond bijectively to a special set of points $\tau \in \mathfrak{H}$. More precisely, there is a bijection between the following two sets:

1. $\operatorname{Hom}^{\sharp}(K, D)=\{$ normalized embeddings $\rho: K \hookrightarrow D\}$.

2. $\mathrm{CM}_{\Delta, K}=\left\{\tau \in \mathfrak{H}\right.$ such that $\left.\operatorname{Stab}(\tau) \cap \Phi_{\infty}\left(D^{\times}\right) \simeq K^{\times}\right\}$.

The bijection is $\Gamma_{0}^{\Delta}(N)$-equivariant where $\Gamma_{0}^{\Delta}(N)$ acts by conjugation on the first set and on $\mathrm{CM}_{\Delta, K}$ via its action on $\mathfrak{H}$. Under the correspondence $\rho \leftrightarrow \tau$ the complex structure on $D_{\infty}$ induced by the embedding $\rho$ coincides with that of $D_{\infty}^{\tau}$. In the split case $\mathrm{CM}_{1, K}=K \cap \mathfrak{H}$.

The conductor $c_{\rho}=c_{\rho}(N)$ relative to the order $\mathcal{R}_{N}$ of the embedding $\rho \in$ $\operatorname{Hom}(K, D)$ is the unique $c \in \mathbb{Z}^{>0}$ such that $\rho\left(\mathcal{O}_{K, c}\right)=\rho(K) \cap \mathcal{R}_{N}$. The conductor $c=c_{\rho}$ of the embedding associated to the point $\tau \in \mathrm{CM}_{\Delta, K}$ depends only on the point $x_{\tau}=[\tau] \in \Gamma_{0}^{\Delta}(N) \backslash \mathfrak{H} \subset X_{0}^{\Delta}(N)$, which will be called a CM point of type $K$ and conductor $c$. We let $\mathrm{CM}(\Delta, N ; K, c)$ denote the set of CM points of type $K$ and conductor $c$. When $\operatorname{gcd}(c, N \Delta)=1$ the $\operatorname{set} \operatorname{CM}(\Delta, N ; K, c)$ is non-empty if and only if all primes $\ell \mid \Delta$ are inert in $K$ and all primes $\ell \mid N$ are split in $K$.

The abelian variety $A_{x}$ corresponding to $x \in \mathrm{CM}_{\Delta, K}$ has a large set of endomorphisms. When $\Delta=1, A$ is a elliptic curve with complex multiplications in $K$. When $\Delta>1$ the QM-abelian surface $A$ contains the CM-elliptic curve $E=K \otimes \mathbb{R} / \mathcal{O}_{K, c_{1}}$ and is in fact isogenous to the product $E \times E$. 
Definition 1.2. Suppose $\Delta>1$. Let $p$ be a prime number, $\operatorname{gcd}(p, 2 \Delta)=1$. A $p$-ordinary triple for $(\Delta, N, K)$ is a triple $(x, v, e)$, where $x \in \mathrm{CM}(\Delta, N ; K, c)$, $v$ is a finite place dividing $p$ in a finite extension $L \supseteq \mathbb{Q}$ and $e \in D \otimes F$ is the idempotent associated to a real quadratic subfield $F \subset D$ pointwise fixed by the positive involution, such that

1. $F K \subseteq L$ and $x_{\tau} \in \mathcal{X}_{1}^{\Delta}(N)(L)$;

2. the QM-abelian surface $A_{x}$ has ordinary good reduction modulo v;

3. if $w$ is the restriction of $v$ to $F$ then $e \in \mathcal{R} \otimes_{\mathbb{Z}} \mathcal{O}_{(w)}$.

Furthermore, a p-ordinary test triple $(x, v, e)$ is said to be split if $p$ splits in $F$, and is said to be arithmetic if $x \in \mathcal{X}_{a r}^{\Delta}(N)\left(\mathcal{O}_{v}\right)$.

Remark 1.3. $\quad$ 1. The ordinarity hypothesis implies that $p$ splits in $K$. We shall denote $\mathfrak{p} \subset \mathcal{O}_{K}$ the prime ideal corresponding to the place of $K$ defined by the restriction of $v$, so that $p \mathcal{O}_{K}=\mathfrak{p} \overline{\mathfrak{p}}$ where $\overline{\mathfrak{p}}$ is the other ideal of $\mathcal{O}_{K}$ of norm $p$.

2. The third condition is equivalent to $\left(p, c_{F} \delta_{F}\right)=1$, where $c_{F}$ is the conductor of $F \subset D$ relative to $\mathcal{R}$.

3. In the split case $(\Delta=1)$ the field $F$ and the idempotent $e$ play no role and only pairs $(x, v)$ will be relevant, where $x \in \mathrm{CM}\left(1, N ; \mathcal{O}_{K, c}\right)$ and $v \mid p$ is some place of ordinary good reduction for the elliptic curve $E_{x}$ in the number field $L$. Speaking indifferently of either case, the term p-ordinary (split, arithmetic) object shall be used.

Proposition 1.4. Let $p$ be a prime number such that $(p, 2 \Delta)=1$. There exists a real quadratic field $F$ in which $p$ splits and an embedding $F \hookrightarrow D$ of conductor prime to $p$ whose image is pointwise fixed by the involution $(\cdot)^{\dagger}$.

Proof. Since maximal orders in $D$ are conjugated, it is enough to work with the Hashimoto model [13] of $(D, \mathcal{R}, t)$, namely

$$
\left\{\begin{array}{l}
D=\mathbb{Q} \oplus \mathbb{Q} i \oplus \mathbb{Q} j \oplus \mathbb{Q} i j, \quad i^{2}=-\Delta, \quad j^{2}=q \\
\mathcal{R}=\mathbb{Z} \oplus \mathbb{Z} \frac{1+j}{2} \oplus \mathbb{Z} \frac{i+i j}{2} \oplus \mathbb{Z} \frac{b \Delta j+i j}{q}
\end{array}\right.
$$

and $t=i$, where $q$ is an auxiliary prime such that $q \equiv 1 \bmod 4(q \equiv 5 \bmod 8$ when $2 \mid \Delta$ ) and $(q,-\Delta)_{\ell}=-1$ if and only if $\ell \mid \Delta$ (such prime exists for Dirichlet's theorem of primes in arithmetic sequence), and $b^{2} \Delta \equiv-1 \bmod q$. Imposing further that $\left(\frac{q}{p}\right)=1$, the request is met by $F=\mathbb{Q}(\sqrt{q})$ embedded as $\sqrt{q} \hookrightarrow j \in \mathcal{R}$, for which $c_{F}=1$. 


\subsection{Differential operators.}

Let $T$ be a scheme, $S$ a smooth $T$-scheme and $\pi: \mathcal{A} \rightarrow S$ an abelian scheme with 0 -section $e_{0}$ and dual $\mathcal{A}_{/ S}^{t}$. Let $\underline{\omega}=\underline{\omega}_{\mathcal{A} / S}=\pi_{*} \Omega_{\mathcal{A} / S}^{1}=e_{0}{ }^{*} \Omega_{\mathcal{A} / S}^{1}$ be the sheaf of translation invariant relative 1 -forms on $\mathcal{A}$ and $\mathcal{H}_{\mathrm{dR}}^{1}=\mathcal{H}_{\mathrm{dR}}^{1}\left(\mathcal{A}_{/ S}\right)=\mathbb{R}^{1} \pi_{*}\left(\Omega_{\mathcal{A} / S}^{\bullet}\right)$ the (first) de Rham sheaf. They are two of the terms in the exact sequence

$$
0 \longrightarrow \underline{\omega} \longrightarrow \mathcal{H}_{\mathrm{dR}}^{1} \longrightarrow R^{1} \pi_{*} \mathcal{O}_{\mathcal{A}} \longrightarrow 0 .
$$

of sheaves on $S$ (the Hodge sequence). The Kodaira-Spencer map

$$
\mathrm{KS}: \underline{\omega} \longrightarrow R^{1} \pi_{*}\left(\pi^{*} \Omega_{S / T}^{1}\right) \simeq \Omega_{S / T}^{1} \otimes R^{1} \pi_{*} \mathcal{O}_{\mathcal{A}} .
$$

is the first boundary map in the long exact sequence arising by pushing down the canonical exact sequence $0 \rightarrow \pi^{*} \Omega_{S / T}^{1} \rightarrow \Omega_{\mathcal{A} / T}^{1} \rightarrow \Omega_{\mathcal{A} / S}^{1} \rightarrow 0$. By Serre duality, it can be seen as an element of $\operatorname{Hom}_{\mathcal{O}_{S}}\left(\underline{\omega}_{\mathcal{A} / S} \otimes \underline{\omega}_{\mathcal{A}^{t} / S}, \Omega_{S / T}^{1}\right)$ and can be reconstructed from the Gauß-Manin connection $\nabla: \mathcal{H}_{\mathrm{dR}}^{1} \rightarrow \mathcal{H}_{\mathrm{dR}}^{1} \otimes_{\mathcal{O}_{S}} \Omega_{S / T}^{1}$ (which is the differential $d_{1}^{0, q}$ in the spectral sequence attached to the filtration $\left.F^{i} \Omega_{\mathcal{A} / T}^{\bullet}=\operatorname{Im}\left(\Omega_{\mathcal{A} / T}^{\bullet-i} \otimes_{\mathcal{O}_{\mathcal{A}}} \pi^{*} \Omega_{S / T}^{i} \longrightarrow \Omega_{\mathcal{A} / T}^{\bullet}\right),[23]\right)$ as

$$
\underline{\omega}_{\mathcal{A} / S} \hookrightarrow \mathcal{H}_{\mathrm{dR}}^{1} \stackrel{\nabla}{\longrightarrow} \mathcal{H}_{\mathrm{dR}}^{1} \otimes \Omega_{S / T}^{1} \longrightarrow\left(\underline{\omega}_{\mathcal{A}^{t} / S}\right)^{\vee} \otimes \Omega_{S / T}^{1} .
$$

When $\mathcal{A}_{/ S}$ is principally polarized, the Kodaira-Spencer map is a symmetric map KS: $\operatorname{Sym}^{2}(\underline{\omega}) \rightarrow \Omega_{S / T}^{1}$ under the identification $\mathcal{A}_{/ S} \simeq \mathcal{A}_{/ S}^{t}$, [6, §III. 9].

When $T=\operatorname{Spec}(\mathbb{C})$ and $S$ is a complex variety the Kodaira-Spencer map of a family $\mathcal{A}_{/ S}$ can be readily computed, appealing to GAGA principles, working in the analytic category as in $[19,8]$. By étale-ness the actual computation can be carried out on the pullback of the family $\mathcal{A}_{/ S}$ on the universal cover of $S$. If $\zeta$ and $\left(\zeta_{1}, \zeta_{2}\right)$ denote the standard complex coordinate in the elliptic curve $E_{z}=\mathbb{C} / \mathbb{Z} \oplus \mathbb{Z} z$ and the QM-abelian surface $A_{z}=\mathbb{C}^{2} / \Phi_{\infty}(\mathcal{R})\left(\begin{array}{c}z \\ 1\end{array}\right)$ respectively, the following formulae for the Kodaira-Spencer map of the universal families over $\mathfrak{H}$ hold:

$$
\begin{aligned}
\mathrm{KS}\left(d \zeta^{\otimes 2}\right) & =\frac{1}{2 \pi i} d z \\
\mathrm{KS}\left(d \zeta_{i} \otimes d \zeta_{j}\right)_{i, j=1,2} & =\frac{1}{2 \pi i}\left(\begin{array}{cc}
1 & 0 \\
0 & \Delta
\end{array}\right) d z
\end{aligned}
$$

Formula (6a) is just the simplest case of the general formula for the universal family of principally polarized abelian varieties of genus $g$ (e.g. $[8, \S 4.4]$ ). Formula (6b) is obtained in [26, Prop. 2.3] in a similar fashion using the Hashimoto model [13].

When $D$ is split, the Kodaira-Spencer map attached to the universal elliptic curve over the Zariski open subscheme $\mathcal{Y}_{\text {ar }}(N)$, complement of the cusp divisor $C$ in $\mathcal{X}_{\text {ar }}^{1}(N)$ is an isomorphism KS: $\underline{\omega}^{\otimes 2} \stackrel{\sim}{\longrightarrow} \Omega_{\mathcal{Y}_{\text {ar }}(N)}^{1}$. It is well known that the line bundle $\underline{\omega}$ extends uniquely at the cusps in the complete curve $\mathcal{X}_{1}^{1}(N)$ and the Kodaira-Spencer isomorphism extends to an isomorphism

$$
\mathrm{KS}: \underline{\omega}^{\otimes 2} \stackrel{\sim}{\longrightarrow} \Omega_{\mathcal{X}_{\mathrm{ar}}^{1}(N)}^{1}(\log C)
$$


(see [18] and also $[22, \S 10.13]$ where the extension property is discussed for a general representable moduli problem). By flat base change

$$
H^{0}\left(\mathcal{X}_{\mathrm{ar}}^{1}(N), \underline{\omega}^{\otimes k}\right) \otimes_{\mathbb{Z}} \mathbb{Z}[1 / N] \stackrel{\sim}{\longrightarrow} H^{0}\left(\mathcal{X}_{1}^{1}(N)_{/ \mathbb{Z}[1 / N]}, \underline{\omega}^{\otimes k}\right)
$$

and $f(z) \mapsto f^{*}=f(z)(2 \pi i d \zeta)^{\otimes k}$ defines an identification

$$
M_{1, k}^{1}(N) \stackrel{\sim}{\longrightarrow} H^{0}\left(X_{1}^{1}(N), \underline{\omega}^{\otimes k}\right) \simeq H^{0}\left(\mathcal{X}_{\mathrm{ar}}^{1}(N), \underline{\omega}^{\otimes k}\right) \otimes \mathbb{C}
$$

normalized so that for a subring $B \subseteq \mathbb{C}$ the modular forms defined over $B$, i.e. the sections in $H^{0}\left(\mathcal{X}_{\mathrm{ar}}^{1}(N) / B, \underline{\omega}^{\otimes k}\right)$ correspond to modular forms with Fourier coefficients in $B$ ( $q$-expansion principle, e.g. [18] [8, theorem 4.8]).

If $D$ is not split, the Kodaira-Spencer map attached to the universal QMabelian surface over $\mathcal{X}_{\mathrm{ar}}^{\Delta}(N)$ has a big kernel. Let $p$ be a prime, $(p, \Delta)=1$, and $v$ be a place in a number field $L$ dividing $p$. The algebra $\mathcal{R} \otimes_{\mathbb{Z}} \mathcal{O}_{(v)}$ has a natural $\mathcal{O}_{(v)}$-linear action on $\underline{\omega}$ over $\mathcal{X}_{\text {ar }}^{\Delta}(N) / \mathcal{O}_{(v)}$. If $e \in \mathcal{R} \otimes_{\mathbb{Z}} \mathcal{O}_{(v)}$ is a nontrivial idempotent, the subsheaf $e \underline{\omega}$ is a line bundle because for any geometric point $s \in \mathcal{X}_{\operatorname{ar}}^{\Delta}(N) / \mathcal{O}_{(v)}$ the decomposition

$$
H^{0}\left(A_{s}, \Omega_{A_{s} / k(s)}^{1}\right)=e H^{0}\left(A_{s}, \Omega_{A_{s} / k(s)}^{1}\right) \oplus(1-e) H^{0}\left(A_{s}, \Omega_{A_{s} / k(s)}^{1}\right)
$$

is non-trivial. Then, [26, Theorem 2.5], the Kodaira-Spencer map restricts to an isomorphism of line bundles

$$
\mathrm{KS}: e \underline{\omega} \circ e^{\dagger} \underline{\omega} \stackrel{\sim}{\longrightarrow} \Omega_{\mathcal{X}_{\text {ar }}^{\Delta}(N) / \mathcal{O}_{(v)}}^{1} .
$$

Notation 1.5. Denote $\mathcal{L}$ either the line bundle $\underline{\omega}$ on $\mathcal{X}_{\text {ar }}^{1}(N) / \mathcal{O}_{(v)}$ or the line bundle $e \underline{\omega}$ on $\mathcal{X}_{\operatorname{ar}}^{\Delta}(N) / \mathcal{O}_{(v)}$ for some choice of idempotent $e \in \mathcal{R}_{1} \otimes_{\mathbb{Z}} \mathcal{O}_{(v)}$ with $v$ as above and such that $e^{\dagger}=e$. In either case the Kodaira-Spencer map gives an isomorphism $\mathrm{KS}: \mathcal{L}^{\otimes 2} \stackrel{\sim}{\longrightarrow} \Omega^{1}$.

The complex line bundle base change of $\mathcal{L}^{\otimes k}$ to the Riemann surfaces $Y_{1}(N)$ or $X_{1}^{\Delta}(N)$ can be constructed also by taking the quotient of the trivial complex line bundle $\mathcal{V}_{k}=\mathfrak{H} \times \mathbb{C}$, homogeneous for the action $g \cdot(z, \zeta)=\left(g \cdot z, j(g, z)^{k} \zeta\right)$ of $\mathrm{GL}_{2}^{+}(\mathbb{R})$, by $\Gamma_{1}^{\Delta}(N)$ respectively. Fix a global constant non-zero section $v_{k} \in H^{0}\left(\mathfrak{H}, \mathcal{V}_{k}\right)$ and let $\varsigma(z) \in H^{0}(\mathfrak{H}, \mathcal{L})$ such that $\operatorname{KS}\left(\varsigma(z)^{\otimes 2}\right)=2 \pi i d z$ (the section $\varsigma(z)$ is given in (6a) in the split case and can be computed explicitly in the non-split case, see [26, Remark 2.6.3]). The identifications over $\mathfrak{H}$

$$
\mathcal{V}_{2} \stackrel{\sim}{\longrightarrow} \Omega^{1}, v_{2} \mapsto 2 \pi i d z \quad \text { and } \quad \mathcal{V}_{k} \stackrel{\sim}{\longrightarrow} \mathcal{L}^{\otimes k}, v_{k} \mapsto \varsigma(z)^{\otimes k}
$$

descend to isomorphisms of complex line bundles on $Y_{1}(N)$ or $X_{1}^{\Delta}(N)$ that preserve holomorphic sections and are compatible with tensor products and the Kodaira-Spencer isomorphisms. Thus, mapping $f(z) \mapsto f^{*}=f(z) \varsigma(z)^{\otimes k}$ allows to identify

$$
M_{1, k}^{\Delta}(N) \stackrel{\sim}{\longrightarrow} H^{0}\left(X_{1}^{\Delta}(N), \mathcal{L}^{\otimes k}\right) \simeq H^{0}\left(\mathcal{X}_{\mathrm{ar}}^{\Delta}(N) / \mathcal{O}_{(v)}, \mathcal{L}^{\otimes k}\right) \otimes \mathbb{C}
$$


(note that there is a sign ambiguity in the $k$ odd case), and say that the modular form $f$ is defined over a ring $B$ of definition for $\mathcal{L}$ if the corresponding section is in $H^{0}\left(\mathcal{X}_{1}^{\Delta}(N)_{/ B}, \mathcal{L}^{\otimes k}\right)$.

Katz [19] pointed out that in a situation where the Hodge sequence (4) admits a splitting $\operatorname{Pr}: \mathcal{H}_{\mathrm{dR}}^{1} \rightarrow \underline{\omega}$ an operator $\Theta_{k, \operatorname{Pr}}: \operatorname{Sym}^{k}(\underline{\omega}) \longrightarrow \operatorname{Sym}^{k}(\underline{\omega}) \otimes \mathcal{L}$ can be defined as the composition

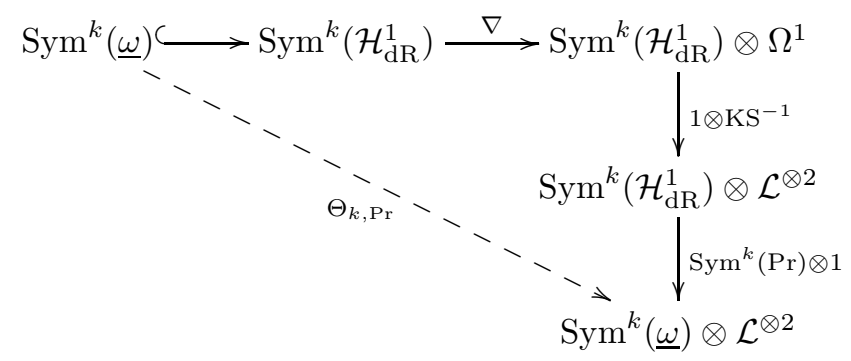

At least two such situations occur after a suitable modification of the basis $S=\mathcal{X}_{\mathrm{ar}}^{\Delta}(N)$ with its universal family.

- Classical (archimedean) Maass operators. If $T=\operatorname{Spec}(\mathbb{C})$, consider the associated differentable manifold $X_{1}^{\Delta}(N)^{\text {an }}$ and denote $\mathcal{M}_{\infty}$ the sheaf of $\mathcal{C}^{\infty}\left(X_{1}^{\Delta}(N)^{\text {an }}\right)$-modules associated to the algebraic sheaf $\mathcal{M}$. The Hodge decomposition $\left(\mathcal{H}_{\mathrm{dR}}^{1}\right)_{\infty}=\underline{\omega}_{\infty} \oplus \underline{\underline{\omega}}_{\infty}$ defines a splitting $\operatorname{Pr}_{\text {Hodge }}$ : $\left(\mathcal{H}_{\mathrm{dR}}^{1}\right)_{\infty} \rightarrow \underline{\omega}_{\infty}$ and ultimately an operator

$$
\Theta_{k, \infty}: \mathcal{L}_{\infty}^{\otimes k} \longrightarrow \mathcal{L}_{\infty}^{\otimes k+2}
$$

([26, Prop. 2.8] for the details about the non-split case). The operator $\Theta_{k, \infty}$ is the classical one-dimensional Maass operator

$$
\delta_{k}=\frac{1}{2 \pi i}\left(\frac{d}{d z}+\frac{k}{2 i y}\right), \quad z=x+y i,
$$

in disguise, since under (7) there is a commutative diagram

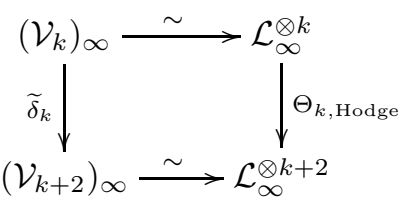

where $\widetilde{\delta}_{k}\left(\phi v_{k}\right)=\delta_{k}(\phi) v_{k+2}$, [26, Prop. 2.9], i.e.

$$
\Theta_{k, \infty}\left(f(z) \varsigma(z)^{\otimes k}\right)=\delta_{k}(f)(z) \varsigma(z)^{\otimes k+2} .
$$

- $p$-adic Maass operators. Let $B$ be a $p$-adic algebra, $(p, \Delta)=1$, such that $e$ is defined over $B$. Let $\mathcal{O}^{[p]}$ be the structure sheaf of the (possibly 
formal) smooth scheme $\mathcal{X}^{[p]}=\lim _{n}\left(\mathcal{X}_{\mathrm{ar}}^{\Delta}(N) / B / p^{n} B\right)^{p \text {-ord }}$ obtained taking out at the finite steps the non-ordinary points in characteristic $p$. Note that if $p \mid N$ then $\mathcal{X}^{[p]}=\mathcal{X}_{\text {ar }}^{\Delta}(N)_{/ B}$ since $\mathcal{X}_{\text {ar }}^{\Delta}(N)$ does not contain nonordinary points in this case. Denote $\mathcal{M}^{[p]}$ the tensorization with $\mathcal{O}^{[p]}$ of the restriction to $\mathcal{X}^{[p]}$ of a sheaf $\mathcal{M}$ on $\mathcal{X}$.

The Dwork-Katz construction [18, §A2.3] of the unique Frobenius-stable $\nabla$-horizontal submodule $\mathcal{U} \subset \mathcal{H}_{\mathrm{dR}}^{1} \otimes B$ can be carried out in the non split case as well. It defines a splitting $\left(\mathcal{H}_{\mathrm{dR}}^{1}\right)^{[p]}=\underline{\omega}^{[p]} \oplus \mathcal{U}$ with projection $\operatorname{Pr}_{\text {Frob }}:\left(\mathcal{H}_{\mathrm{dR}}^{1}\right)^{[p]} \rightarrow \underline{\omega}^{[p]}$ and ultimately an operator

$$
\Theta_{k, p}:\left(\mathcal{L}^{\otimes k}\right)^{[p]} \longrightarrow\left(\mathcal{L}^{\otimes k+2}\right)^{[p]}
$$

(see [26] for the details about the non-split case)

The operators $\Theta_{k, *}$ can be iterated by increasing $k$ : for all $r \geq 1$ let $\Theta_{k, *}^{(r)}=$ $\Theta_{k+2 r-2, *} \circ \cdots \circ \Theta_{k, *}$. The operators $\Theta_{k, *}^{(r)}$ are algebraic over the CM locus in the following sense. Let $x \in \operatorname{CM}(\Delta, N, K, c)$ in some arithmetic $p$-ordinary test object, with corresponding abelian variety $A=A_{x}$. The choice of an invariant form $\omega_{o}$ on $A_{x}$ which generates either $H^{0}\left(A, \Omega_{A / \mathcal{O}_{(v)}}^{1}\right)$ in the split case or $e H^{0}\left(A, \Omega_{A / \mathcal{O}_{(v)}}\right)$ in the non-split case identifies the algebraic fiber $\mathcal{L}(x)=x^{*} \mathcal{L}$ with a copy of $\mathcal{O}_{(v)}$. Then, for all $r \geq 1$ the operator $\Theta_{k, *}^{(r)}$ define a map

$$
\Theta_{k, *}^{(r)}(x): H^{0}\left(\mathcal{X}_{\mathrm{ar}}^{\Delta}(N) / \mathcal{O}_{(v)}, \mathcal{L}^{\otimes k}\right) \longrightarrow \mathcal{L}^{\otimes k+2 r}(x) \simeq \mathcal{O}_{(v)} \omega_{o}{ }^{\otimes k+2 r}
$$

and in fact

$$
\Theta_{k, \infty}^{(r)}(x)\left(f^{*}\right)=\Theta_{k, p}^{(r)}(x)\left(f^{*}\right)
$$

for a modular form $f$ defined over $\mathcal{O}_{(v)}$. As in [20, theorem 2.4.5] this follows from the fact that both the Hodge decomposition of $\mathcal{H}_{\mathrm{dR}}^{1}\left(A_{/ \mathbb{C}}\right)$ and the Dwork-Katz decomposition of $\mathcal{H}_{\mathrm{dR}}^{1}\left(A_{/ B}\right)$ for a $p$-adic $\mathcal{O}_{(v)}$-algebra $B$ can be obtained by a suitable tensoring from the eigenspace decomposition of the algebraic $\mathcal{H}_{\mathrm{dR}}^{1}\left(A_{/ \mathcal{O}_{(v)}}\right)$ under the action of the complex multiplications in $K$. Also, (11) holds for all $r \geq 1$ because $\Theta_{k, *}^{(r)}=\operatorname{Pr}_{*}\left(\left(1 \otimes K S^{-1}\right) \nabla\right)^{r}$, since the kernels of $\operatorname{Pr}_{*}$ are $\nabla$-horizontal. For all $r \geq 1$ write $\delta_{k}^{(r)}=\delta_{k+2 r-2} \circ \cdots \circ \delta_{k}$.

\section{Expansions and measures}

\subsection{Serre-Tate Theory}

Let $\mathbf{k}$ be a field of characteristic $p>0, \Lambda$ a complete local ring with residue field $\mathbf{k}, \mathcal{C}_{\Lambda}$ the category of artinian local $\Lambda$-algebras with residue field $\mathbf{k}$ and $\mathbf{A} / \mathbf{k}$ an abelian variety of dimension $g$. Grothendieck has shown that the local moduli functor

$$
\mathcal{M}_{\mathbf{A}}: \mathcal{C}_{\Lambda} \rightarrow \text { Sets, } \quad \mathcal{M}_{\mathbf{A}}(B)=\left\{\begin{array}{c}
\text { Abelian schemes } \mathcal{A}=\mathcal{A} / B \\
\text { such that } \mathcal{A} \otimes_{\Lambda} \mathbf{k}=\mathbf{A}
\end{array}\right\}
$$


is pro-represented by $\Lambda\left[\left[t_{1}, \ldots, t_{g^{2}}\right]\right]$. The Serre-Tate theory makes precise the fact that a deformation of $\mathbf{A}$ to $B \in \mathcal{C}_{\Lambda}$ is completely determined by a deformation of its formal group. When $\mathbf{k}$ is algebraically closed, $\Lambda=\mathcal{W}$ is a finite extension of the ring $W_{\mathbf{k}}$ of Witt vectors and $\mathbf{A}$ is ordinary, all of which shall be assumed henceforth, there is a canonical isomorphism of functors

$$
\mathcal{M}_{\mathbf{A}} \stackrel{\sim}{\longrightarrow} \operatorname{Hom}\left(T_{p} \mathbf{A} \otimes T_{p} \mathbf{A}^{t}, \widehat{\mathbb{G}}_{m}\right),
$$

which endows $\mathcal{M}_{\mathbf{A}}=\operatorname{Spf}\left(\mathfrak{R}^{u}\right)$ with a canonical structure of formal torus and identifies its group of characters $X\left(\mathcal{M}_{\mathbf{A}}\right)=\operatorname{Hom}_{\mathbb{Z}_{p}}\left(\mathcal{M}_{\mathbf{A}}, \widehat{\mathbb{G}}_{m}\right) \subset \mathfrak{R}^{u}$ with $T_{p} \mathbf{A} \otimes$ $T_{p} \mathbf{A}^{t}$. For a deformation $\mathcal{A}_{/ B}$ of $\mathbf{A}$, let

$$
q(\mathcal{A} / B ; \cdot, \cdot): T_{p} \mathbf{A} \times T_{p} \mathbf{A}^{t} \longrightarrow \widehat{\mathbb{G}}_{m}(B)=1+\mathfrak{m}_{B}
$$

be the corresponding bilinear form. Briefly, $q\left(\mathcal{A}_{B} ; P, Q^{t}\right)=\left\langle\varphi_{\mathcal{A}}(P), Q^{t}\right\rangle$ (essentially a Weil pairing) where $\varphi_{\mathcal{A}}: T_{p}(\mathbf{A}) \rightarrow \widehat{\mathcal{A}}$ is the limit of the Drinfeld lifts of the multiplication by $p^{n}$ maps and realizes the canonical connected-étale exact sequence for the $p$-divisible group of $\mathcal{A}$ as the pushout of the standard exact sequence for $T_{p}(\mathbf{A})$,

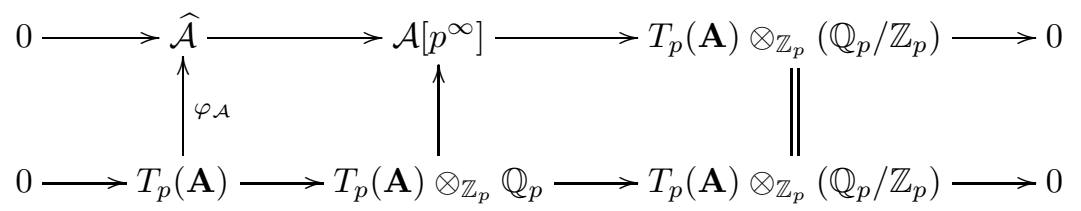

Grothendieck's result is thus explicit, in the sense that if $T_{p}(\mathbf{A})=\bigoplus_{i=0}^{g} \mathbb{Z}_{p} P_{i}$ and $T_{p}\left(\mathbf{A}^{t}\right)=\bigoplus_{j=0}^{g} \mathbb{Z}_{p} P_{j}^{t}$, and $\mathcal{A}_{/ \Re^{u}}^{u}$ is the universal formal deformation, the $g^{2}$ elements

$$
q_{i, j}=q\left(\mathcal{A}_{/ \mathfrak{R}^{u}}^{u} ; P_{i}, P_{j}^{t}\right)-1=\lim _{\overparen{B \in \mathcal{C}}} q\left(\mathcal{A}_{/ B} ; P_{i}, P_{j}^{t}\right)-1
$$

define an isomorphism $\mathfrak{R}^{u} \simeq \mathcal{W}\left[\left[q_{i, j}\right]\right]$. The following two facts (for, and for the details of all of the above as well, see [21]) are crucial.

Remark 2.1. If $\mathcal{A}_{/ B}$ and $\mathcal{B}_{/ B}$ are formal deformations of the ordinary abelian varieties $\mathbf{A}$ and $\mathbf{B}$ respectively, a morphism $f: \mathbf{A} \rightarrow \mathbf{B}$ lifts to a morphism $f_{B}: \mathcal{A} \rightarrow \mathcal{B}$ if and only if

$$
q\left(\mathcal{A}_{/ B} ; P, f^{t}\left(Q^{t}\right)\right)=q\left(\mathcal{B}_{/ B} ; f(P), Q^{t}\right) \quad \text { for all } P \in T_{p} \mathbf{A}, Q^{t} \in T_{p} \mathbf{B}^{t} .
$$

In particular, if $\mathbf{A}$ is principally polarized, under the identification $T_{p} \mathbf{A} \simeq T_{p} \mathbf{A}^{t}$ the formal subscheme $\mathcal{M}_{\mathbf{A}}^{\mathrm{pp}}$ that classifies deformations of $\mathbf{A}$ with a lifting of the principal polarization is a subtorus with group of characters $X\left(\mathcal{M}_{\mathbf{A}}^{\mathrm{pp}}\right)=$ $\operatorname{Sym}^{2}\left(T_{p} \mathbf{A}\right)$. 
Remark 2.2. The Weil pairing yields an isomorphism $T_{p} \mathbf{A}^{t} \simeq \operatorname{Hom}_{B}\left(\widehat{\mathcal{A}}, \widehat{\mathbb{G}}_{m}\right)$, hence a functorial $\mathbb{Z}_{p}$-linear homomorphism $\omega_{\mathcal{A}}: T_{p} \mathbf{A}^{t} \rightarrow \underline{\omega}_{\mathcal{A} / B}$, where $\omega_{\mathcal{A}}\left(P^{t}\right)$ is the pullback of $d t / t$ under the morphism corresponding to $P^{t}$. Thus, if $\mathbf{A}$ is principally polarized and $\mathcal{A}_{/ B}$ is a principally polarized deformation

$$
\omega_{\mathcal{A}}(P) \cdot \mathrm{KS}\left(\omega_{\mathcal{A}}(Q)\right)=d \log q\left(\mathcal{A}_{/ B} ; P, Q\right) \quad \text { for all } P, Q \in T_{p} \mathbf{A}
$$

under the usual identifications.

By ordinarity, $\mathbf{A}[p]=\widehat{\mathbf{A}}[p] \times\left(T_{p}(\mathbf{A}) \otimes_{\mathbb{Z}_{p}} \mathbb{F}_{p}\right)$. The two factors are mutually Cartier dual finite group schemes, each of them giving rise to a quotient isogeny of degree $p^{g}$. The connected factor is the kernel of the completely inseparable k-linear Frobenius morphism $F_{\mathbf{k}}: \mathbf{A} \rightarrow \mathbf{A}^{(p)}$. Denoting $\mathbf{A}^{(1 / p)}$ the quotient of A by the étale factor, the separable isogeny $G: \mathbf{A} \rightarrow \mathbf{A}^{(1 / p)}$ is the Verschiebung for $\mathbf{A}^{(1 / p)}$ because $\left(\mathbf{A}^{(1 / p)}\right)^{(p)} \simeq \mathbf{A}$, i.e. $G^{(p)}=V_{\mathbf{k}}$.

Proposition 2.3. Let $\mathbf{A}$ be endowed with a polarization $\lambda$ of degree not divisible by $p$. Then there are identifications $T_{p}(\mathbf{A}) \simeq T_{p}\left(\mathbf{A}^{t}\right) \simeq T_{p}\left(\mathbf{A}^{(p)}\right) \simeq$ $T_{p}\left(\left(\mathbf{A}^{(p)}\right)^{t}\right) \simeq T_{p}\left(\mathbf{A}^{(1 / p)}\right) \simeq T_{p}\left(\left(\mathbf{A}^{(1 / p)}\right)^{t}\right)$ under which a morphism $\phi: \mathcal{A} \rightarrow \mathcal{B}$ of abelian schemes over $B \in \widehat{\mathcal{C}}$ lifts the Frobenius morphism $F_{\mathbf{k}}$ if and only if

$$
q(\mathcal{B} ; P, Q)=q(\mathcal{A} ; P, Q)^{p} \quad \text { for all } P, Q \in T_{p}(\mathbf{A}),
$$

and a morphism $\psi: \mathcal{A} \rightarrow \mathcal{B}$ of abelian schemes over $B \in \widehat{\mathcal{C}}$ lifts $G$ if and only if

$$
q(\mathcal{B} ; P, Q)^{p}=q(\mathcal{A} ; P, Q) \quad \text { for all } P, Q \in T_{p}(\mathbf{A}),
$$

Proof. Since ker $\lambda$ meets $\mathbf{A}[p]$ trivially, there are canonically induced polarizations $\lambda^{(p)}$ on $\mathbf{A}^{(p)}$ and $\lambda^{(1 / p)}$ on $\mathbf{A}^{(1 / p)}$ such that $\operatorname{deg}(\lambda)=\operatorname{deg}\left(\lambda^{(p)}\right)=$ $\operatorname{deg}\left(\lambda^{(1 / p)}\right)$. Thus, the situation defines a (non-commutative!) diagram of Tate modules

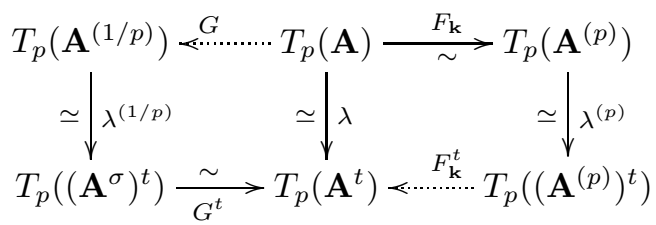

where the continuous arrows are isomorphisms $\left(G^{t}\right.$ is a $\mathbf{k}$-linear Frobenius, as observed above) and the dotted arrows are, under these identifications, multiplication by $p$ maps. The result follows directly from (12).

If $\mathbf{E}_{/ \mathbf{k}}$ is an elliptic curve, the local moduli functor $\mathcal{M}_{\mathbf{E}}^{1}=\operatorname{Spf}\left(\Re^{u}\right)$ is a onedimensional torus with $\mathfrak{R}^{u}=\mathcal{W}[[q-1]]$ where $q=q\left(\mathcal{E}_{/ \mathfrak{R}^{u}}^{u} ; P, P\right)$ and $T_{p}(\mathbf{E})=$ $\mathbb{Z}_{p} P$. Also, $\operatorname{KS}\left(\omega_{u}^{\otimes 2}\right)=d \log (q)$ where $\omega_{u}=\omega_{\mathcal{E}^{u}}(P)$.

For a QM-abelian surface $\mathbf{A}_{/ \mathbf{k}}$ a choice of idempotent $e \in \mathcal{R} \otimes \mathbb{Z}_{p} \simeq \mathrm{M}_{2}\left(\mathbb{Z}_{p}\right)$ defines a decomposition $T_{p}(\mathbf{A})=\mathbb{Z}_{p} P \oplus \mathbb{Z}_{p} Q$ of Tate modules where $e P=P$ 
and $e Q=0$. Since QM-abelian surfaces have a canonical principal polarization it is natural to consider the subfunctor

$\mathcal{M}_{\mathbf{A}}^{\Delta}(B)=\left\{\begin{array}{c}\text { principally polarized deformations } \mathcal{A}_{/ B} \text { of } \mathbf{A} \text { with a lift of the } \\ \text { endomorphisms given by elements of the maximal order } \mathcal{R}\end{array}\right\}$.

Then, [26, Proposition 3.3],

Proposition 2.4. The subfunctor $\mathcal{M}_{\mathbf{A}}^{\Delta}=\operatorname{Spf}\left(\Re_{D}^{u}\right)$ is a 1-dimensional subtorus of $\mathcal{M}_{\mathbf{A}}$. Moreover, if $e \in \mathcal{R} \otimes \mathbb{Z}_{p}$ is an idempotent such that $e^{\dagger}=e$ and $\{P, Q\}$ is a $\mathbb{Z}_{p}$-basis of $T_{p}(\mathbf{A})$ such that $e P=P$ and $e Q=0$, then

1. $\operatorname{Spf}\left(\mathfrak{R}_{D}^{u}\right)=\mathcal{W}[[q-1]]$, where $q=\left.q\left(\mathcal{A}^{u} ; P, P\right)\right|_{\mathcal{M}_{\mathbf{A}}^{\Delta}} ;$

2. $\mathrm{KS}\left(\omega_{u}^{\otimes 2}\right)=d \log q$, where $\omega_{u}=\left.\omega_{\mathcal{A}^{u}}(P)\right|_{\mathcal{M}_{\mathbf{A}}^{\Delta}}$.

Remark 2.5. Let $\varepsilon \in \mathcal{R} \otimes \mathbb{Z}_{p}$ such that $\varepsilon P=Q, \varepsilon Q=P$, and let $\mathcal{A} \in \mathcal{M}_{\mathbf{A}}^{\Delta}(B)$. Then, by continuity of the action of $\mathcal{R}$ on $T_{p}(\mathbf{A})$,

$$
\left.q(\mathcal{A} ; P, Q)\right|_{\mathcal{M}_{\mathrm{A}}^{\Delta}}=\left.q(\mathcal{A} ; e P, Q)\right|_{\mathcal{M}_{\mathrm{A}}^{\Delta}}=\left.q(\mathcal{A} ; P, e Q)\right|_{\mathcal{M}_{\mathrm{A}}^{\Delta}}=1
$$

and since $\varepsilon^{\dagger}$ acts on $\{P, Q\}$ as $\left(\begin{array}{rr}0 & \rho \\ 1 / \rho & 0\end{array}\right)$ for some $\rho \in \mathbb{Z}_{p}^{\times}$,

$$
\left.q(\mathcal{A} ; Q, Q)\right|_{\mathcal{M}_{\mathbf{A}}^{\Delta}}=\left.q(\mathcal{A} ; \varepsilon P, Q)\right|_{\mathcal{M}_{\mathbf{A}}^{\Delta}}=\left.q\left(\mathcal{A} ; P, \varepsilon^{\dagger} Q\right)\right|_{\mathcal{M}_{\hat{\mathbf{A}}}^{\Delta}}=\left(\left.q(\mathcal{A} ; P, P)\right|_{\mathcal{M}_{\mathbf{A}}^{\Delta}}\right)^{\rho}
$$

Thus for a morphism $f: \mathbf{A}_{1} \rightarrow \mathbf{A}_{2}$ of QM-abelian surfaces to lift to a morphism of QM deformations is enough that $q\left(\mathcal{A}_{2} ; f\left(P_{1}\right), P_{2}\right)=q\left(\mathcal{A}_{1} ; P_{1}, f^{t}\left(P_{2}\right)\right)$ where $P_{1} \in T_{p}\left(\mathbf{A}_{1}\right)$ and $P_{2} \in T_{p}\left(\mathbf{A}_{2}\right)$ are as in proposition 2.4.

Let $\mathbf{A}_{/ \mathbf{k}}$ be either an elliptic curve or a QM-abelian surface and $P \in T_{p}(\mathbf{A})$ either a $\mathbb{Z}_{p}$-generator or as in proposition 2.4 respectively. Let $\mathcal{A} \rightarrow \mathcal{B}$ a lift of the k-linear Frobenius over $B \in \widehat{\mathcal{C}}$ and let $q_{0}=q(\mathcal{A} ; P, P)$.

Proposition 2.6. Let $\Delta \geq 1$. The following two sets are in bijection:

1. the formal deformations $\mathcal{A}^{\prime} \in \mathcal{M}_{\mathbf{A}}^{\Delta}(B)$ such that $q\left(\mathcal{A}^{\prime} ; P, P\right) \in q_{0} \mu_{p}(B)$;

2. the étale subgroups $C$ of $\mathcal{B}[p]$ of rank $p^{\operatorname{dim}(\mathbf{A})}$ which are $\mathcal{R}$-invariant when $D$ is non split.

Proof. An immediate consequence of proposition 2.3 is that up to the identification $\left(\mathbf{A}^{(1 / p)}\right)^{(p)} \simeq \mathbf{A}$ the first set is the set of deformations $\mathcal{A}^{\prime} \in \mathcal{M}_{\mathbf{A}}^{\Delta}$ for which there is a lift $\mathcal{A}^{\prime} \rightarrow \mathcal{B}$ of the $\mathbf{k}$-linear Frobenius for $\mathbf{A}$ and also those for which there is a lift $\mathcal{B} \rightarrow \mathcal{A}^{\prime}$ of $V_{\mathbf{k}}=G^{(p)}: \mathbf{A}^{(p)} \rightarrow \mathbf{A}$. On the other hand, if $\psi: \mathcal{B} \rightarrow \mathcal{A}^{\prime}$ lifts $V_{\mathbf{k}}$, the subgroup $\operatorname{ker}(\psi)<\mathcal{B}[p]$ is in the second set. Finally, if $C$ is in the second set, the quotient $\mathcal{B} / C$ is a deformation of $\mathbf{A}$ and the quotient map lifts $V_{\mathbf{k}}$. 
The bijection can be made explicit as follows. The deformation $\mathcal{B}$ can be recovered from the universal deformation $\mathcal{B}^{u}$ of $\mathbf{A}^{(p)}$ as pullback via the classifying map $\phi_{\mathcal{B}}: \mathcal{W}[[q-1]] \rightarrow B$ such that $\phi_{\mathcal{B}}(q-1)=q_{0}^{p}-1$. Since $\phi_{\mathcal{B}}$ factors through the map $\alpha: \mathcal{W}[[q-1]] \rightarrow \mathcal{W}[[q-1]]$ such that $\alpha(q)=q^{p}$ there is a diagram of pullbacks

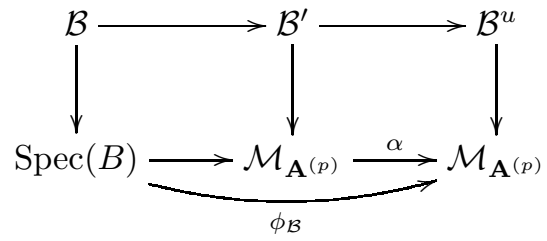

and a map $\mathcal{A}^{u} \rightarrow \mathcal{B}^{\prime}$ which is a lift of $F_{\mathbf{k}}$. Let $C<\mathcal{B}[p]$ as in the second set of proposition 2.6. Let $\widetilde{C}$ an étale subgroup of $\mathcal{B}^{u}$ such that $C=\widetilde{C} \times{ }_{\phi_{\mathcal{B}}} B$ and let $C^{\prime}=\widetilde{C} \times{ }_{\alpha} \mathcal{M}_{\mathbf{A}^{(p)}}$. Also, let $\mathcal{B}_{/ B}^{\text {can }}$ be the pullback of $\mathcal{B}^{u}$ (or $\left.\mathcal{B}^{\prime}\right)$ via the map $q \mapsto 1$ and $C^{\text {can }}$ the pullback of $\widetilde{C}$ (or $C^{\prime}$ ). The deformation $\mathcal{B}^{\text {can }}$ is the canonical lift of $\mathbf{A}^{(p)}$, characterized by $q\left(\mathcal{B}^{\text {can }} ; P, P\right)=1$ or, equivalently by the splitting of the connected-étale exact sequence of its $p$-divisible group, i.e. $\mathcal{B}^{\text {can }}\left[p^{\infty}\right]=\widehat{\mathcal{B}}^{\text {can }} \times T_{p}\left(\mathbf{A}^{(p)}\right) \otimes\left(\mathbb{Q}_{p} / \mathbb{Z}_{p}\right)$. Up to a choice of isomorphism $\widehat{\mathcal{B}}^{\text {can }}[p]=\mu_{p}(B)^{\operatorname{dim} \mathbf{A}}$ (where for QM-abelian surfaces the factors are chosen so that the action of $e$ is projection onto the first) write $C^{\text {can }}=\left\langle\left(\zeta, P_{1}\right)\right\rangle$ for some $\zeta \in \mu_{p}(B)$ (such that $e \zeta=\zeta$ when $\operatorname{dim}(\mathbf{A})=2$ ), where $P=\lim P_{n}$. Since the quotient isogeny $\mathcal{B}^{\text {can }} \rightarrow \mathcal{B}^{\text {can }} / C^{\text {can }}$ induces an isomorphism of formal groups, the connected-étale exact sequence for $\left(\mathcal{B}^{\text {can }} / C^{\text {can }}\right)\left[p^{\infty}\right]$,

$$
0 \rightarrow \widehat{\mathcal{B}}^{\text {can } / C^{\text {can }}} \longrightarrow \frac{\widehat{\mathcal{B}}^{\text {can }} \times T_{p}\left(\mathbf{A}^{(p)}\right) \otimes\left(\mathbb{Q}_{p} / \mathbb{Z}_{p}\right)}{\left\langle\left(\zeta, P_{1}\right)\right\rangle} \longrightarrow T_{p}(\mathbf{A}) \otimes\left(\mathbb{Q}_{p} / \mathbb{Z}_{p}\right) \rightarrow 0,
$$

arises as pushout for the map $\varphi_{\mathcal{B}^{\text {can }}} / C^{\text {can }}(P)=\zeta^{-1}$. Thus,

$$
\frac{q(\mathcal{B} / C ; P, P)}{q(\mathcal{A} ; P, P)}=\frac{q\left(\mathcal{B}^{\mathrm{can}} / C^{\mathrm{can}} ; P, P\right)}{q\left(\mathcal{A}^{\mathrm{can}} ; P, P\right)}=\zeta^{-1}
$$

by the connectedness of the local moduli space.

\subsection{Expansions}

Let $(p, M)=1$ and $\mathcal{T}_{\nu}=\left(x_{\nu}, v_{\nu}, e\right), \nu \geq 0$, an arithmetic $p$-ordinary (split if $\Delta>1)$ object for $\left(\Delta, M p^{\nu}, K\right)$. The abelian variety $A$ corresponding to $x_{\nu}$ has a smooth model $A_{v}$ over $\mathcal{O}_{(v)}$ with $v \mid v_{\nu}$ equipped with an arithmetic level structure $\eta_{M p^{\nu}}$ of level $M p^{\nu}$. Let $\mathbf{k}=\bar{k}_{v}$ and $\mathbf{A}=A_{v} \otimes \mathbf{k}$ the reduction of $A$ modulo $v$ base changed to k. Let $\mathcal{W} \subseteq \mathcal{W}_{\nu}$ be finite extensions of $W_{\mathbf{k}}$ containing $\mathcal{O}_{v}$ and $\mathcal{O}_{v_{\nu}}$ respectively. By smoothness, the local moduli space $\mathcal{M}_{\mathbf{A}}^{\Delta}\left(\mathcal{W}_{\nu}\right)=\operatorname{Spf}\left(\Re^{u} \otimes \mathcal{W}_{\nu}\right)$ is the formal completion of $\mathcal{X}_{\text {ar }}^{\Delta}\left(M p^{\nu}\right) / \mathcal{W}_{\nu}$ along $x_{\nu}$ and there is a canonical isomorphism

$$
\mathfrak{R}^{u} \otimes \mathcal{W}_{\nu} \simeq \widehat{\mathcal{O}}_{\mathcal{X}_{\text {ar }}\left(M p^{\nu}\right)_{/ \mathcal{W}_{\nu}}, x_{\nu}},
$$


limit of the classifying maps $\mathfrak{R}^{u} \otimes \mathcal{W}_{\nu} \rightarrow \mathcal{O}_{x_{\nu}} / \mathfrak{m}_{x_{\nu}}^{n}$ that correspond to the restriction of the universal family to $\operatorname{Spec}\left(\mathcal{O}_{x_{\nu}} / \mathfrak{m}_{x_{\nu}}^{n}\right)$. This identification allows to extend the arithmetic structure $\eta_{M p^{\nu}}$ to $\mathcal{A}_{/ \mathfrak{R}^{u} \otimes \mathcal{W}_{\nu}}^{u}$. Fix $P \in T_{p}(\mathbf{A})$ as in proposition 2.4 and the discussion preceding it and let $q=q\left(\mathcal{A}^{u} ; P, P\right)$ and $\underline{\omega}_{u}=\underline{\omega}_{\mathcal{A}^{u}}(P)$. Letting $q_{0}=q\left(A_{v}\right) \in 1+\mathfrak{p}_{\mathcal{W}}$, it is also

$$
\widehat{\mathcal{O}}_{\mathcal{X}_{\mathrm{ar}}\left(M p^{\nu}\right) / \mathcal{W}_{\nu}, x_{\nu}} \simeq \mathcal{W}_{\nu}\left[\left[q_{x}\right]\right] \quad \text { where } q_{x}=q_{0}^{-1} q-1
$$

The parameter $q_{x}$ is a canonical local formal parameter "centered at $q_{0}$ ". It is canonical in the sense that it is the pullback of the Serre-Tate parameter $q-1$ under the translation by $q_{0}^{-1}$ in the formal torus $\mathcal{M}_{\mathbf{A}}^{\Delta}$.

Let $f \in M_{1, k}^{\Delta}\left(M p^{\nu} ; \mathcal{O}_{\left(v_{\nu}\right)}\right)$ a modular form defined over $\mathcal{O}_{\left(v_{\nu}\right)}$. The corresponding section $f^{*} \in H^{0}\left(\mathcal{M}_{\mathbf{A}}^{\Delta}, \mathcal{L}^{\otimes k}\right)$ at $x_{\nu}$ can be written $f^{*}=f_{u} \omega_{u}^{\otimes k}$ with $f_{u} \in \mathfrak{R}^{u} \otimes \mathcal{W}_{\nu}$. Thus, the identification (13) allows to write an expansion

$$
f_{u}=F_{f}\left(q_{x}\right)=\sum_{n=0}^{\infty} a_{n}\left(f, x_{\nu}\right) q_{x}^{n}
$$

We shall also write

$$
f_{u}=\Phi_{f}\left(Q_{x}\right)=\sum_{n=0}^{\infty} \frac{b_{n}\left(f, x_{\nu}\right)}{n !} Q_{x}^{n}=F_{f}\left(e^{Q_{x}}-1\right)
$$

under the formal substitution $Q_{x}=\log \left(1+q_{x}\right)=q_{x}-\frac{1}{2} q_{x}^{2}+\frac{1}{3} q_{x}^{3}-\cdots$.

Remark 2.7. It turns out that $b_{n}\left(f, x_{\nu}\right) \in \mathcal{W}_{\nu}$ for all $n \geq 0$. Moreover, if the coefficients $\gamma_{n, i} \in \mathbb{Z}$ are defined by the identity $n !\left(\begin{array}{c}X \\ n\end{array}\right)=\sum_{i=0}^{n} \gamma_{n, i} X^{i}$, then

$$
\frac{1}{n !} \sum_{i=1}^{n} \gamma_{n, i} b_{i}\left(f, x_{\nu}\right) \in \mathcal{W}_{\nu} \quad \text { for all } n \geq 1
$$

Remark 2.8. The choice of $P \in T_{p}(\mathbf{A})$ induces also a trivialization

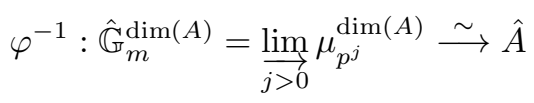

i.e. a compatible sequence of arithmetic structures $\eta_{p^{\nu}}$ on $A$ which together with a structure $\eta_{M}$ of level $M$ gives rise to a sequence $\mathcal{T}_{j}=\left(x_{j}, v_{j}, e\right), j \geq 0$, of arithmetic $p$-ordinary (split if $\Delta>1$ ) objects for $\left(\Delta, M p^{j}, K\right)$ which are compatible for the canonical forgetful maps $\mathcal{X}_{\mathrm{ar}}^{\Delta}\left(M p^{j+1}\right) \rightarrow \mathcal{X}_{\mathrm{ar}}^{\Delta}\left(M p^{j}\right)$. The expansion (14) remains the same if the object $\mathcal{T}_{\nu}$ is replaced with $\mathcal{T}_{\nu^{\prime}}$ for all $\nu \leq \nu^{\prime}$. Let $\mathcal{W}_{\infty}=\lim \mathcal{W}_{j}$ and let $\varphi^{u}: \widehat{\mathcal{A}}^{u} \stackrel{\sim}{\longrightarrow} \widehat{\mathbb{G}}_{m}\left(\Re^{u} \otimes \mathcal{W}_{\infty}\right)^{\operatorname{dim} A}$ and $\eta_{M}^{u}$ be the unique extension of $\varphi$ and $\eta_{M}$ to the formal universal deformation respectively. Then we can write

$$
F_{f}\left(q_{x}\right)=f\left(\mathcal{A}^{u}, \varphi^{u}, \eta_{M}^{u}\right)
$$

where $f$ is reinterpreted as a generalized $p$-adic modular function in the sense of Katz (for the extension of Katz's theory to quaternionic forms, see [17]). 
To a choice of an invariant form $\omega_{o}$ on $A_{v}$ normalized as in (10) are attached periods as follows.

- Archimedian period. Fix a complex uniformization of $A_{/ \mathbb{C}}$, which amounts to choosing a suitable $\tau \in \mathfrak{H}$. For a given $\omega \in H^{0}\left(A_{\tau}, \mathcal{L}(\tau)\right)$ write $\omega=$ $\operatorname{per}_{\infty}(\omega, \tau) \varsigma(\tau)$ with $\operatorname{per}_{\infty}(\omega, \tau) \in \mathbb{C}$ and let

$$
\Omega_{\infty}=\operatorname{per}_{\infty}\left(\omega_{o}, \tau\right)
$$

- $p$-adic period. For $\omega \in H^{0}\left(A_{/ \mathcal{W}}, \mathcal{L}\right)$ write $\omega=\operatorname{per}_{p}(\omega, P) \omega_{u}(P)_{\mid A}$ with $\operatorname{per}_{p}(\omega, P) \in \mathcal{W}$ and let

$$
\Omega_{p}=\operatorname{per}_{p}\left(\omega_{o}, P\right) \in \mathcal{W}^{\times} .
$$

In turn, the archimedean period can be used to define numbers

$$
\theta_{v}^{(r)}(f, x)=\theta^{(r)}\left(f, x, \omega_{o}\right) \in \mathbb{C}
$$

where $\theta^{(r)}\left(f, x_{\nu}, \omega\right)=\operatorname{per}_{\infty}(\omega, \tau)^{-k-2 r} \delta_{k}^{(r)}(f)(\tau)$ for any $\omega \in H^{0}\left(A_{\tau}, \mathcal{L}(\tau)\right)$. These numbers depend only on $x$ and not on the choice of a representant $\tau$. The following result relates the periods to the expansion (14).

Theorem 2.9. Let $f \in M_{1, k}^{\Delta}\left(M p^{\nu} ; \mathcal{O}_{\left(v_{\nu}\right)}\right)$ and $x \in \mathcal{X}_{a r}^{\Delta}\left(M p^{\nu}\right)\left(\mathcal{O}_{\left(v_{\nu}\right)}\right)$ belonging to a p-ordinary arithmetic test object (split, if $\Delta>1$ ). Then

$$
\theta_{v}^{(r)}(f, x)=\Omega_{p}^{-k-2 r} b_{r}(f, x) \in \mathcal{O}_{\left(v_{\nu}\right)} \quad \text { for all } r \geq 0 .
$$

Proof. The result is obvious for $r=0$, so that $r \geq 1$ may be assumed. Since $\operatorname{Pr}_{\text {Frob }}\left(\nabla\left(\omega_{u}\right)\right)=0$, a consequence of $\left[21\right.$, theorem 4.3.1] is that $\Theta_{k, p}(f)=$ $\left(d f_{u} / d Q_{x}\right) \omega_{u}^{\otimes k+2}$. After $r$ iterations and evaluation at $x$,

$$
\Theta_{k, p}^{(r)}(f)(x)=\frac{d^{r} f_{u}}{d Q_{x}{ }^{r}}(x) \omega_{u}^{k+2 r}(x)=\frac{b_{r}(f, x)}{\Omega_{p}^{k+2 r}} \omega_{o}^{k+2 r} .
$$

On the other hand, (9) yields

$$
\Theta_{k, \infty}^{(r)}(f)(x)=\delta_{k}^{(r)}(f)(\tau) s(\tau)^{\otimes k+2 r}=\theta_{v}^{(r)}(f, x) \omega_{o}^{k+2 r} .
$$

The result follows from (11).

By the classical theory of Mahler the series $F_{f}$ in (14), defines a a $\mathcal{W}_{\nu}$-valued measure $\mu_{f, x_{\nu}}$ on $\mathbb{Z}_{p}$ characterized by $\int_{\mathbb{Z}_{p}}\left(\begin{array}{l}t \\ r\end{array}\right) d \mu_{f, x_{\nu}}(t)=a_{r}\left(f, x_{\nu}\right)$ for all $r \geq 0$. Also, the moments of $\mu_{f, x_{\nu}}$ can be read off the expansion (15), namely

$$
m_{r}\left(\mu_{f, x_{\nu}}\right)=\int_{\mathbb{Z}_{p}} t^{r} d \mu_{f, x_{\nu}}(t)=b_{r}\left(f, x_{\nu}\right)=\Omega_{p}^{k+2 r} \theta_{v}^{(r)}\left(f, x_{\nu}\right) \quad \text { for all } r \geq 0 .
$$

Remark 2.10. By theorem 2.9 the measure $\mu_{f, x_{\nu}}$ takes values in the field $L_{v_{\nu}}\left(\Omega_{p}\right)$, which is well-defined since $\Omega_{p}$ is well-defined as an element of $\mathcal{W}^{\times} / \mathcal{O}_{v}^{\times}$. 
Denote $\mu^{\times}$the restriction of the measure $\mu$ to $\mathbb{Z}_{p}^{\times}$, i.e. $\int_{\mathbb{Z}_{p}^{\times}} \phi d \mu^{\times}=\int_{\mathbb{Z}_{p}} \phi^{*} d \mu$ where $\phi^{*}$ is the function obtained from $\phi$ setting it equal to 0 on $p \mathbb{Z}_{p}$. The following theorem shows how the operation of restricting $\mu_{f, x_{\nu}}$ to $\mathbb{Z}_{p}^{\times}$is strongly related to the action of the $p$-th Hecke operator on $f$. The operators $U$ and $V$ in the statement denote the classical operators (for which the explicit dependence on $p$ will be dropped).

Theorem 2.11. Let $f \in M_{1, k}^{\Delta}\left(M p^{\nu} ; \mathcal{O}_{\left(v_{\nu}\right)}\right)$. Then $\mu_{f, x_{\nu}}^{\times}=\mu_{(1-V U) f, x_{\nu+1}}$.

Proof. The general theory says that the formal power series encoding $\mu_{f, x_{\nu}}^{\times}$is

$$
F_{f}^{\times}(T)=F_{f}(T)-\frac{1}{p} \sum_{\zeta \in \mu_{p}} F_{f}(\zeta T+\zeta-1) .
$$

Following remark 2.8 we read the summands in the right hand side (after evaluation at $T=q_{x}$ ) as values of $f$ at particular trivialized abelian schemes over $\mathcal{W}_{\infty}\left[\left[q_{x}\right]\right]$. Given a map of $\mathcal{W}_{\infty}$-algebras $h: \mathcal{W}_{\infty}\left[\left[q_{x}\right]\right] \rightarrow B$ there is an equality $h\left(F_{f}\left(q_{x}\right)\right)=f\left(\mathcal{A}^{h}, \varphi^{h}, \eta_{\nu}^{h}\right)$ where the triple $\left(\mathcal{A}^{h}, \varphi^{h}, \eta_{M}^{h}\right)$ is obtained from $\left(\mathcal{A}^{u}, \varphi^{u}, \eta_{M}^{u}\right)$ by pull-back:

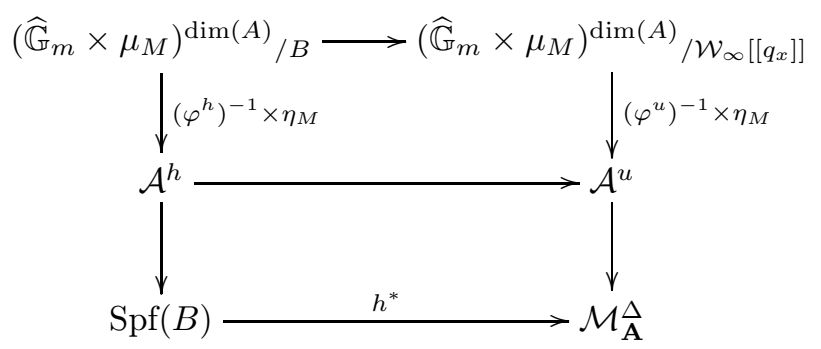

Thus,

$$
F_{f}^{\times}\left(q_{x}\right)=f\left(\mathcal{A}^{u}, \phi^{u}, \eta_{M}^{u}\right)-\frac{1}{p} \sum_{\zeta \in \mu_{p}} f\left(\mathcal{A}^{\zeta}, \phi^{\zeta}, \eta_{M}^{\zeta}\right)
$$

where the triple $\left(\mathcal{A}^{\zeta}, \phi^{\zeta}, \eta_{\nu}^{\zeta}\right)$ is obtained as above from the endomorphism of $\mathcal{W}_{\infty}\left[\left[q_{x}\right]\right]$ determined by $q_{x} \mapsto \zeta q_{x}+\zeta-1$. Since $\zeta q_{x}+\zeta-1=q_{0}^{-1}(\zeta q)-1$, by proposition 2.6 the sum extends over the set $\mathcal{F}$ of deformations $\mathcal{A} / \mathcal{W}_{\infty}\left[\left[q_{x}\right]\right]$ admitting a lift $\pi_{\mathcal{A}}: \mathcal{A} \rightarrow \mathcal{A}^{u, p}=\mathcal{A}^{u} / H^{u}$ of the k-linear Frobenius $\left(H^{u}<\mathcal{A}^{u}\right.$ the canonical subgroup). On the other hand, if $U_{p}$ and $V_{p}$ denote the $p$-adic counterparts of the classic $U$ and $V$ operators (e.g. [7]),

$$
V_{p} U_{p} f\left(\mathcal{A}^{u}, \varphi^{u}, \eta_{M}^{u}\right)=U_{p} f\left(\mathcal{A}^{u, p}, \varphi^{u, p}, \eta_{M}^{u, p}\right)=\frac{1}{p} \sum_{\mathcal{A} \in \mathcal{F}} f\left(\mathcal{A}, \phi^{\mathcal{A}}, \eta_{M}^{\mathcal{A}}\right)
$$

where $\varphi^{u, p}=\varphi^{u} \pi_{\mathcal{A}^{u}}^{t}, \eta_{M}^{u, p}=\pi_{\mathcal{A}^{u}} \mu_{M}^{u}$ and for each $\mathcal{A} \in \mathcal{F}, \varphi^{\mathcal{A}}=\varphi^{u} \pi_{A^{u}}^{t}\left(\pi_{\mathcal{A}}^{t}\right)^{-1}$ and $\eta_{M}^{\mathcal{A}}=\pi_{\mathcal{A}}^{-1} \pi_{\mathcal{A}^{u}} \eta_{M}^{u}$ (the notation is justified because each lift of Frobenius is an isomorphism between $M$-torsion subgroups and its dual is ètale and an isomorphism between formal groups). Since $\varphi^{\mathcal{A}}=\varphi^{\zeta}$ and $\eta_{\nu}^{\mathcal{A}}=\eta_{\nu}^{\zeta}$ when $\mathcal{A}=\mathcal{A}^{\zeta}$ 
because the lifts of Frobenius commute with the pull-back map (16) this proves that $\mu_{f, x_{\nu}}^{\times}=\mu_{\left(1-V_{p} U_{p}\right) f, x_{\nu}}$.

To finish the proof, observe that for $f \in M_{1, k}^{\Delta}\left(M p^{\nu} ; \mathcal{O}_{\left(v_{\nu}\right)}\right)$ and for every triple $\left(\mathcal{A}, \varphi, \eta_{M}\right)$ defined over a $p$-adic ring $U_{p} f\left(\mathcal{A}, \varphi, \eta_{M}\right)=U f\left(\mathcal{A}, \eta_{M} \times\right.$ $\left.\varphi_{\mid \mu_{p^{\nu}}}^{-1}, \omega_{\mathcal{A}}(\varphi)\right)$ and $V_{p} f\left(\mathcal{A}, \varphi, \eta_{M}\right)=V f\left(\mathcal{A}, \eta_{M} \times \varphi_{\mid \mu_{p^{\nu}}}^{-1}, \omega_{\mathcal{A}}(\varphi)\right)$ because of the compatibility of the $p$-part of the level structures, where $\left.\omega_{\mathcal{A}}(\varphi)\right)$ is the unique extension to $\mathcal{A}$ of the invariant 1 -form defined by the trivialization (see remarks 2.2 and 2.8$)$.

\subsection{More measures}

A weight for the imaginary quadratic imaginary field $K$ is a formal linear combination $\underline{w}=\left(w_{1}, w_{\sigma}\right)=w_{1}$ id $+w_{\sigma} \sigma \in \mathbb{Z}[\operatorname{Gal}(K / \mathbb{Q})]$. Write $\lambda \underline{w}=\lambda^{w_{1}} \sigma(\lambda)^{w_{\sigma}}$ for all $\lambda \in K$. For an ideal $\mathfrak{n} \subset \mathcal{O}_{K}$, a weight $\underline{w}$ and a field $K \subseteq E$ let

$$
H_{\underline{w}}(\mathfrak{n} ; E)=\left\{\phi: \mathcal{I}_{\mathfrak{n}} \rightarrow E \text { such that } \phi((\lambda) I)=\lambda \stackrel{\underline{w}}{\phi}(I) \text { for all } \lambda \in K_{\mathfrak{n}}\right\}
$$

(Hida's $K^{\times}$-modular forms of level $\mathfrak{n}$, weight $\underline{w}$ and coefficients in $E$, [14]). From an observation of Weil in [32] one knows that the algebraic Hecke characters in $H_{\underline{w}}(\mathfrak{n} ; \mathbb{C})$ are in $H_{\underline{w}}(\mathfrak{n} ; \overline{\mathbb{Q}})$. When $E \subseteq \overline{\mathbb{Q}}$, there are local avatars for the spaces $\overline{H_{\underline{w}}}(\mathfrak{n} ; E)$. Denote $\overline{\mathfrak{n}}^{*}$ the prime to $p$ part of $\mathfrak{n}$.

- Archimedean avatars. Denote $H_{\underline{w}}^{(\infty)}(\mathfrak{n})$ the space of $\mathbb{C}$-valued functions on $K_{\mathbb{A}}^{\times}$such that

$$
\Phi(t \lambda z u)=z^{-\underline{w}} \Phi(t) \quad \text { for all } \lambda \in K^{\times}, z \in \mathbb{C}^{\times}, u \in U_{\mathfrak{n}}
$$

where $z^{-\underline{w}}=z^{-w_{1}} \bar{z}^{-w_{\sigma}}$ (the restriction of $\Phi$ to $\mathbb{C}$ is the infinity type of $\Phi)$. Given $\phi \in H_{\underline{w}}(\mathfrak{n} ; E)$ there exists a unique $\phi^{(\infty)} \in H_{\underline{w}}^{(\infty)}(\mathfrak{n})$ such that $\phi^{(\infty)}(t)=h(I)$ whenever $t_{v}=1$ for all $v \mid \infty \mathfrak{n}$ and $I=[t]$. The mapping $\phi \mapsto \phi^{(\infty)}$ extends by linearity to an isomorphism $H_{\underline{w}}(\mathfrak{n} ; \mathbb{C})=$ $H_{\underline{w}}(\mathfrak{n} ; \overline{\mathbb{Q}}) \otimes \mathbb{C} \simeq H_{\underline{w}}^{(\infty)}(\mathfrak{n})$.

- $p$-adic avatars. Let $F \supset E$ be a $p$-adic local subfield of $\mathbb{C}_{p}$ and $\mathfrak{F}\left(\mathfrak{n}^{*} ; F\right)$ the space of $F$-valued continuous functions on $\mathfrak{C}_{\mathfrak{n}^{*}}=\mathfrak{C}_{\mathfrak{n}}=\lim _{j \geq 0} \mathbf{C}_{\mathfrak{n} p^{j}}$. Then, every $\phi \in H_{\underline{w}}\left(\mathfrak{n}^{*} ; E\right)$ defines a unique $\phi^{(p)} \in \mathfrak{F}\left(\mathfrak{n}^{*} ; F\right)$ which agrees with $\phi$ on the dense subset $\mathcal{I}_{\mathfrak{n}^{*} p}$. The mapping $\phi \mapsto \phi^{(p)}$ identifies $H_{\underline{w}}\left(\mathfrak{n}^{*} p^{r} ; E\right) \otimes$ $F$ with the closed subspace $\mathfrak{F}_{\underline{w}, r}\left(\mathfrak{n}^{*} ; F\right) \subset \mathfrak{F}\left(\mathfrak{n}^{*} ; F\right)$ of functions such that $\Phi(x t)=x^{-\underline{w}} \Phi(t)$ for all $x \in \overline{\mathcal{O}}_{K} \otimes \mathbb{Z}_{p}, x \equiv 1 \bmod \mathfrak{n}^{*} p^{r}$.

Note that $\xi \in H_{\underline{w}}(\mathfrak{n} ; \overline{\mathbb{Q}})$ is a character if and only if its avatars $\xi^{(\infty)}$ and $\xi^{(p)}$ are characters. Let

$$
H(\mathfrak{n} ; E)=\bigoplus_{\underline{w} \in \mathbb{Z}[\operatorname{Gal}(K / \mathbb{Q})]} H_{\underline{w}}(\mathfrak{n} ; E) .
$$

If $\mathfrak{m} \mid \mathfrak{n}$ then $H(\mathfrak{m} ; E) \subset H(\mathfrak{n} ; E)$ and by the linear independence of characters the construction of the $p$-adic avatars embeds $H(\mathfrak{n} \mathfrak{q} ; E) \otimes F$ in $\mathfrak{F}\left(\mathfrak{n}^{*} ; F\right)$ when 
$\mathfrak{q}$ is supported on primes dividing $p$. The space $H(\mathfrak{n} ; E)$ supports an $E$-valued pairing defined as follows. If $\phi_{1} \in H_{\underline{w}_{1}}(\mathfrak{n} ; E)$ and $\phi_{2} \in H_{\underline{w}_{2}}(\mathfrak{n} ; E)$ let

$$
\left\langle\phi_{1}, \phi_{2}\right\rangle=\frac{1}{h_{\mathfrak{n}}} \sum_{s \in \mathbf{C}_{\mathfrak{n}}} \phi_{1}\left(I_{s}\right) \phi_{2}\left(I_{s}\right), \quad \text { if } \underline{w}_{1}+\underline{w}_{2}=\underline{0}
$$

and $\left\langle\phi_{1}, \phi_{2}\right\rangle=0$ otherwise, where $\left\{I_{s}\right\}$ is a full set of representatives of $\mathbf{C}_{\mathfrak{n}}$. Then, extend to the whole of $H(\mathfrak{n} ; E)$ by linearity. Note that the pairing is compatible with the inclusions $H(\mathfrak{n} ; E) \subset H\left(\mathfrak{n}^{\prime} ; E\right)$ for $\mathfrak{n} \mid \mathfrak{n}^{\prime}$ and that the characters satisfy the orthogonality relations $\left\langle\xi_{1}, \xi_{2}\right\rangle=1$ if $\xi_{1} \xi_{2}=1$ and $\left\langle\xi_{1}, \xi_{2}\right\rangle=0$ otherwise. The pairing can be computed in terms of the avatars.

- Archimedean pairing. For all $\Phi_{1} \in H_{\underline{w}_{1}}^{(\infty)}(\mathfrak{n})$ and $\Phi_{2} \in H_{\underline{w}_{2}}^{(\infty)}(\mathfrak{n})$ let

$$
\left\langle\Phi_{1}, \Phi_{2}\right\rangle_{\infty}=\frac{1}{h_{\mathfrak{n}}} \sum_{s \in \mathbf{C}_{\mathfrak{n}}} \Phi_{1}\left(t_{s}\right) \Phi_{2}\left(t_{s}\right), \quad \text { if } \underline{w}_{1}+\underline{w}_{2}=\underline{0}
$$

and $\left\langle\Phi_{1}, \Phi_{2}\right\rangle_{\infty}=0$ otherwise, where $\left\{t_{s}\right\}$ is a full set of representatives of $\mathbf{C}_{\mathfrak{n}}$ in $K_{\mathbb{A}}^{\times}$. After extending to $H^{(\infty)}(\mathfrak{n})=\bigoplus_{\underline{w}} H_{\underline{w}}^{(\infty)}(\mathfrak{n})$ by linearity, it is clear that $\left\langle\phi_{1}, \phi_{2}\right\rangle=\left\langle\phi_{1}^{\infty}, \phi_{2}^{\infty}\right\rangle_{\infty}$ for all $\phi_{1}, \bar{\phi}_{2} \in H(\mathfrak{n}, E)$ and their corresponding archimedean avatars since it is always possible to take representatives such that $t_{s, v}=1$ for all $v \mid \infty \mathfrak{n}$, thus reducing (19) to (18).

- p-adic pairing. Let $\mathfrak{F}_{r}\left(\mathfrak{n}^{*} ; F\right)=\widehat{\bigoplus}_{\underline{w}} \mathfrak{F}_{\underline{w}, r}\left(\mathfrak{n}^{*} ; F\right)$. Since $\mathfrak{F}_{\underline{w}, r}\left(\mathfrak{n}^{*} ; F\right)$ is closed, the projection onto the $\underline{w}$-th summand extends to a continuous projection $\pi_{\underline{w}, r}: \mathfrak{F}_{r}\left(\mathfrak{n}^{*} ; F\right) \rightarrow \mathfrak{F}_{\underline{w}, r}\left(\mathfrak{n}^{*} ; F\right)$. Define $\langle\cdot, \cdot\rangle_{p}$ as

$$
\mathfrak{F}_{r}\left(\mathfrak{n}^{*} ; F\right) \times \mathfrak{F}_{r}(\underbrace{\left.\mathfrak{n}^{*} ; F\right) \stackrel{m}{\longrightarrow} \mathfrak{F}_{r}\left(\mathfrak{n}^{*} ; F\right) \stackrel{\pi_{0}, r}{\longrightarrow} \mathfrak{F}_{\underline{0}, r}\left(\mathfrak{n}^{*} ; F\right) \stackrel{\mu_{H}}{\longrightarrow} F}_{\langle\cdot, \cdot\rangle_{p}},
$$

where $m$ is multiplication and $\mu_{H}$ the Haar distribution (bounded on $\left.\mathfrak{F}_{\underline{0}, r}(\mathfrak{n} ; F)\right)$. Then $\left\langle\phi_{1}, \phi_{2}\right\rangle=\left\langle\phi_{1}^{(p)}, \phi_{2}^{(p)}\right\rangle_{p}$ for all $\phi_{1}, \phi_{2} \in H(\mathfrak{n}, E)$ because $\mathbf{C}_{n}$ admits representantives in the dense subset $\mathcal{I}_{\mathfrak{n} p}$, where $\phi_{1}=\phi_{1}^{(p)}$ and $\phi_{2}=\phi_{2}^{(p)}$ so that the computation of $\left\langle\phi_{1}, \phi_{2}\right\rangle_{p}$ reduces to (18).

For a continuous character $\chi: \widehat{\mathcal{O}}_{K, c}^{\times} \rightarrow \mathbb{C}^{\times}$, denote $H_{\underline{w}}^{(\infty)}(\chi)$ the linear space of functions $\Phi: K_{\mathbb{A}}^{\times} \rightarrow \mathbb{C}$ such that

$$
\Phi(\lambda t z u)=z^{-\underline{w}} \Phi(t) \chi(u) \quad \text { for all } \lambda \in K^{\times}, z \in \mathbb{C}^{\times}, u \in \widehat{\mathcal{O}}_{K, c^{\circ}}^{\times} .
$$

Let $\mathfrak{n}_{\chi}$ be the smallest ideal such that $H_{\underline{w}}^{(\infty)}(\chi) \subset H_{\underline{w}}^{(\infty)}\left(\mathfrak{n}_{\chi}\right)$. Note that $\mathfrak{n}_{\chi}$ is at least divisible by the primes $\mathfrak{p}$ not in the support of $c$ and such that $\chi_{\mid\left(\mathcal{O}_{K, c}\right)_{\mathfrak{p}}^{\times}} \not \equiv 1$ and may be divisible by primes $\mathfrak{p}$ in the support of $c$ even if $\chi_{\mid\left(\mathcal{O}_{K, c}\right)_{\mathfrak{p}}^{\times}} \equiv 1$. For 
$\Phi_{1} \in H_{\underline{w}_{1}}^{(\infty)}\left(\chi_{1}\right)$ and $\Phi_{2} \in H_{\underline{w}_{2}}^{(\infty)}\left(\chi_{2}\right)$ with $\underline{w}_{1}+\underline{w}_{2}=0$ and $\chi_{1} \chi_{2}=1$ the pairing simplifies to

$$
\left\langle\Phi_{1}, \Phi_{2}\right\rangle_{\infty}=\frac{1}{h_{c}^{\sharp}} \sum_{s \in \mathbf{C}_{c}^{\sharp}} \Phi_{1}\left(t_{s}\right) \Phi_{2}\left(t_{s}\right),
$$

where $\left\{t_{s}\right\}$ is now a full set of representatives of $\mathbf{C}_{c}^{\sharp}$ in $K_{\mathbb{A}}^{\times}$, which we can assume to be $p$-normalized, i.e. $t_{s, \infty}=1, t_{s, \ell} \in \mathcal{O}_{K} \otimes \mathbb{Z}_{\ell}$ for all primes $\ell$ and furthermore $t_{s, v}$ a $v$-unit for all places $v \mid p c$, for all $s \in \mathbf{C}_{c}^{\sharp}$.

Let $\mu_{1}, \mu_{2}$ be measures on $\mathbb{Z}_{p}$ with values in the $p$-adic Banach $F$-space $\mathfrak{B}$. For a continuous function $h: \mathbb{Z}_{p}^{2} \rightarrow \mathbb{Z}_{p}$ let $\mu_{h}=h_{*}\left(\mu_{1} \otimes \mu_{2}\right)$ the $\mathfrak{B} \widehat{\otimes}_{F} \mathfrak{B}$-valued measure on $\mathbb{Z}_{p}$ such that $\int_{\mathbb{Z}_{p}} \varphi(t) d \mu_{h}(t)=\iint_{\mathbb{Z}_{p}^{2}} \varphi\left(h\left(t_{1}, t_{2}\right)\right) d\left(\mu_{1} \otimes \mu_{2}\right)\left(t_{1}, t_{2}\right)$ for all $\varphi \in \mathcal{C}^{0}\left(\mathbb{Z}_{p}, F\right)$. When $h=m$ is the multiplication the moments of the measure $\mu_{m}$ are simply

$$
m_{n}\left(\mu_{m}\right)=\int_{\mathbb{Z}_{p}} t^{n} d \mu_{m}=\iint_{\mathbb{Z}_{p}^{2}}\left(t_{1} t_{2}\right)^{n} d\left(\mu_{1} \otimes \mu_{2}\right)\left(t_{1}, t_{2}\right)=m_{n}\left(\mu_{1}\right) \otimes m_{n}\left(\mu_{2}\right)
$$

for all $n \geq 0$. In particular, if $\mathfrak{B}=F$ and $\mu_{2}=\delta_{z}$ is the Dirac measure at $z \in \mathbb{Z}_{p}$ then $m_{n}\left(\mu_{m}\right)=m_{n}\left(\mu_{1}\right) z^{n}$. Also, $t_{1} t_{2} \in \mathbb{Z}_{p}^{\times}$if and only if $\left(t_{1}, t_{2}\right) \in\left(\mathbb{Z}_{p}^{\times}\right)^{2}$ so that

$$
\mu_{m}^{\times}=m_{*}\left(\mu_{1}^{\times} \otimes \mu_{2}^{\times}\right) .
$$

Proposition 2.12. Let $\mu_{1}$ and $\mu_{2}$ be $\mathfrak{F}\left(\mathfrak{n}^{*} ; F\right)$-valued measures on $\mathbb{Z}_{p}$ such that $m_{n}\left(\mu_{i}\right)=\int_{\mathbb{Z}_{p}} t^{n} d \mu_{i}(t)=\lambda_{n, i} \phi_{n, i}^{(p)}$ for all $n \geq 0$ and $i \in\{1,2\}$, where $\lambda_{n, i} \in F$ and $\phi_{n, i}^{(p)}$ is the $p$-adic avatar of some $\phi_{n, i} \in H_{\underline{w}_{n, i}}(\mathfrak{n} ; E)$ for a subfield $E \subset \mathbb{C}$ admitting an embedding into $F$ and such that $\underline{w}_{n, 1}+\underline{w}_{n, 2}=\underline{0}$. Then there exists an F-valued measure $\mu=\mu_{\left\langle\mu_{1}, \mu_{2}\right\rangle}$ on $\mathbb{Z}_{p}$ such that

$$
m_{n}(\mu)=\int_{\mathbb{Z}_{p}} t^{n} d \mu(t)=\lambda_{n, 1} \lambda_{n, 2}\left\langle\phi_{n, 1}, \phi_{n, 2}\right\rangle .
$$

Moreover, $\mu^{\times}=\mu_{\left\langle\mu_{1}^{\times}, \mu_{2}^{\times}\right\rangle}$.

Proof. Fix $r \geq 0$ such that $\mathfrak{n} \mid \mathfrak{n}^{*} p^{r}$ : it is clear that $\int_{\mathbb{Z}_{p}} \varphi d \mu_{i} \in \mathfrak{F}_{r}\left(\mathfrak{n}^{*} ; F\right)$ for all $\varphi \in \mathcal{C}^{0}\left(\mathbb{Z}_{p}, F\right)$. Since the Haar distribution is bounded on $\mathfrak{F}_{0}\left(\mathfrak{n}^{*} ; F\right)$, the pairing $\langle\cdot, \cdot\rangle_{p}$ is bounded on $\mathfrak{F}_{r}\left(\mathfrak{n}^{*} ; F\right) \times \mathfrak{F}_{r}\left(\mathfrak{n}^{*} ; F\right)$. If $F_{p}$ denotes the bounded linear functional on $\mathfrak{F}_{r}\left(\mathfrak{n}^{*} ; F\right) \widehat{\otimes} \mathfrak{F}_{r}\left(\mathfrak{n}^{*} ; F\right)$ corresponding to $\langle\cdot, \cdot\rangle_{p}$ the setting

$$
\int_{\mathbb{Z}_{p}} \varphi(t) d \mu(t)=F_{p}\left(\iint_{\mathbb{Z}_{p}^{2}} \varphi\left(t_{1} t_{2}\right) d\left(\hat{\mu}_{1} \times \hat{\mu}_{2}\right)\left(t_{1}, t_{2}\right)\right)
$$

defines a measure on $\mathbb{Z}_{p}$ which has the stated properties because of the preceding discussion. 
Remark 2.13. Composing either measure $\mu_{i}$ with the evaluation map at any $I \in \mathcal{I}_{\mathfrak{n}}$ defines $F$-valued measures $\mu_{i}^{I}$ such that $m_{n}\left(\mu_{i}^{I}\right)=\lambda_{n, i} \phi_{n . i}^{(p)}(I)$. Taking a system of representatives of $\mathbf{C}_{\mathfrak{n}}$ in $\mathcal{I}_{\mathfrak{n}^{*} p}$ yields

$$
\begin{aligned}
& \left\langle m_{r}\left(\mu_{1}\right), m_{r}\left(\mu_{2}\right)\right\rangle_{p}= \\
& \quad \frac{\lambda_{r, 1} \lambda_{r, 2}}{h_{\mathfrak{n}}} \sum_{s \in \mathbf{C}_{\mathfrak{n}}} \phi_{r, 1}\left(I_{s}\right) \phi_{r, 2}\left(I_{s}\right)=\frac{1}{h_{\mathfrak{n}}} \sum_{s \in \mathbf{C}_{\mathfrak{n}}} m_{r}\left(m_{*}\left(\mu_{1}^{I_{s}} \times \mu_{2}^{I_{s}}\right)\right) .
\end{aligned}
$$

By additivity of measures, this provides an alternate method of constructing $\mu_{\left\langle\mu_{1}, \mu_{2}\right\rangle}$.

Remark 2.14. Given a character $\psi: \mathbf{C}_{\mathfrak{n}} \rightarrow \mathbb{C}^{\times}$the formula

$$
\left\langle\phi_{1}, \phi_{2}\right\rangle^{\psi}=\left\langle\phi_{1}, \psi \phi_{2}\right\rangle
$$

defines a twisted version of the pairing (18) which has likewise archimedean and $p$-adic counterparts. The same argument of the proof of proposition 2.12 yields a measure $\mu^{\psi}$ on $\mathbb{Z}_{p}$ such that $\int_{\mathbb{Z}_{p}} t^{n} d \mu(t)=\lambda_{n, 1} \lambda_{n, 2}\left\langle\phi_{n, 1}, \phi_{n, 2}\right\rangle^{\psi}$. The measures $\mu^{\psi}$ can be read as a measure $\mu$ on $\mathbf{C}_{\mathfrak{n}} \times \mathbb{Z}_{p}$ such that

$$
\int_{\mathbf{C}_{\mathfrak{n}} \times \mathbb{Z}_{p}} \psi(s) t^{n} d \mu(s, t)=\lambda_{n, 1} \lambda_{n, 2}\left\langle\phi_{n, 1}, \psi \phi_{n, 2}\right\rangle
$$

We shall now define the measure which will be used for the interpolation process in the next section. Let $\rho \in \operatorname{Hom}^{\sharp}(K, D)$ with corresponding point $x_{0}=[\tau] \in \operatorname{CM}\left(\Delta, N ; \mathcal{O}_{K, c}\right)$. Its adelic extension $\rho_{\mathbb{A}}$ gives rise to an inclusion

$$
\bar{\rho}_{\mathbb{A}}: \mathbf{C}_{c}^{\sharp} \longrightarrow D^{\times} \backslash D_{\mathbb{A}}^{\times} / \rho\left(\mathbb{C}^{\times}\right) \widehat{\mathcal{R}}_{N}^{\times} \hookrightarrow X_{0}^{\Delta}(N)
$$

(the last map is actually an equality when $\Delta>1$ ). Under the decomposition

$$
D_{\mathbb{A}}^{\times}=D_{\mathbb{Q}}^{\times} \mathrm{GL}_{2}(\mathbb{R})^{+} \widehat{\mathcal{R}}_{N}^{\times}
$$

the idele $d=d_{\mathbb{Q}} g_{\infty} \hat{r}$ corresponds to the point represented by $g_{\infty} \tau$. The canonical map of class field theory $K_{\AA}^{\times} \longrightarrow \operatorname{Gal}\left(K^{\mathrm{ab}} / K\right)$, where $t \mapsto[t, K]$, yields a canonical identification $\mathbf{C}_{c}^{\sharp} \simeq \operatorname{Gal}\left(H_{c} / K\right)$ where $H_{c}$ is the ring class field of conductor $c$. The points in $\operatorname{Im}\left(\bar{\rho}_{\mathbb{A}}\right)$ are defined over $H_{c}$ and by Shimura's reciprocity law the two Galois actions are compatible. In particular, if $s=[t, K]_{\mid H_{c}}$ then $\bar{\rho}_{\mathbb{A}}(\bar{t})=x_{0}^{s}$ and $A_{x_{0}^{s}}^{s} \simeq A_{x_{0}}$. Also, for a choice of $p$-normalized representatives $\left\{t_{s}\right\}$ the theory of complex multiplications yields isogenies of complex tori of prime to $p$ degree

$$
A_{g_{s} \tau}=\mathbb{C}^{\epsilon} / \Lambda_{g_{s} \tau} \stackrel{j\left(g_{s}, \tau\right)}{\sim} A_{x_{0}^{s}}(\mathbb{C})=\mathbb{C}^{\epsilon} / t_{s} \Lambda_{\tau} \stackrel{\pi_{t_{s}}}{\longrightarrow} A_{x_{0}}(\mathbb{C})=\mathbb{C}^{\epsilon} / \Lambda_{\tau}
$$

$(\epsilon=1$ or 2$)$ where $\pi_{t_{s}}$ is a quotient map. Let $w \mid p$ a place in a large enough field $L$ such that $\left(x^{s}, w, e\right)$ is a $p$-ordinary split arithmetic object for $(\Delta, N, K)$ for all 
$s \in \mathbf{C}_{c}^{\sharp}$ and for all $x^{s}$ mapping to $x_{0}^{s}$ under the natural map $X_{1}^{\Delta}(N) \rightarrow X_{0}^{\Delta}(N)$, and also such that the isogenies $\pi_{t_{s}}$ are defined over $L$. Fix an invariant 1-form $\omega_{o}$ on $A_{x}$ as in (10) and let $\omega_{t_{s}}=\pi_{t_{s}}^{*}\left(\omega_{o}\right)$. Then $\omega_{t_{s}}$ satisfies (10) for $A_{x_{s}}$ so that it may be assumed that

$$
\Omega_{\infty}\left(g_{s} \tau\right)=j\left(g_{s}, \tau\right) \Omega_{\infty}(\tau) \text { and } \Omega_{p}\left(x^{s}\right)=\Omega_{p}(x) .
$$

If $t_{s}^{\prime}=t_{s} \lambda z u$ is another $p$-normalized representative of $s \in \mathbf{C}_{c}^{\sharp}$, then comparing decompositions (21) yields $\omega_{t_{s}^{\prime}} \sim_{\mathcal{O}_{K, c}^{\times}} z \omega_{t_{s}}$. Thus, the construction of $\omega_{t}$ can be extended, up to $\mathcal{O}_{K, c}^{\times}$-association, to all of $K_{\mathbb{A}}^{\times}$by setting $\omega_{t \lambda z u} \sim_{\mathcal{O}_{K, c}^{\times}} z \omega_{t}$ for all $\lambda \in K^{\times}, z \in \mathbb{C}^{\times}, u \in \mathcal{O}_{K, c}^{\times}$where $t$ is $p$-normalized.

Fix a point $x \in X_{1}^{\Delta}(N)$ such that $x \mapsto x_{0}$ under the natural map and assume that $x$ belongs to a $p$-ordinary split arithmetic object (this depends only on $x_{0}$ ). Let $f \in M_{0,2 \kappa}^{\Delta}\left(N, \varepsilon ; \mathcal{O}_{(v)}\right)$. The nebentypus $\varepsilon$ gives rise to a character $\varepsilon_{\mathbb{A}}$ of $\mathcal{R}_{N}^{\times}$ and thus to a character $\varepsilon_{\rho}$ of $\widehat{\mathcal{O}}_{K, c}^{\times}$via the embedding $\rho$. For all $r \geq 0$ such that $\left(\mathcal{O}_{K, c}^{\times}\right)^{2(\kappa+r)}=1$ define a complex valued function $\vartheta^{(\infty)}(r, f, x)$ on $K_{\mathbb{A}}^{\times}$as

$$
\vartheta^{(\infty)}(r, f, x)(t)=\frac{\delta_{2 \kappa}^{(r)} f\left(g_{\infty} \tau\right) \varepsilon_{\mathbb{A}}(\hat{r})}{\operatorname{per}_{\infty}\left(\omega_{t}, g_{\infty} \tau\right)^{2(\kappa+r)}}
$$

where $\rho_{\mathbb{A}}(t)=d_{0} g_{\infty} \hat{r}$ under $(21)$.

Proposition 2.15. $\vartheta^{(\infty)}(r, f, x) \in H_{(2(\kappa+r), 0)}^{(\infty)}\left(\varepsilon_{\rho}\right)$. Moreover, $\vartheta^{(\infty)}(r, f, x)$ is the archimedean avatar of $\vartheta(r, f, x) \in H_{(2(\kappa+r), 0)}\left(\mathfrak{n}_{\varepsilon_{\rho}} ; L\right)$ and its $p$-adic avatar $\vartheta^{(p)}(r, f, x)$ is in $\mathfrak{S}\left(\mathfrak{n}_{\varepsilon_{\rho}}^{*} ; L_{w}\right)$, the unitary ball in $\mathfrak{F}\left(\mathfrak{n}_{\varepsilon_{\rho}}^{*} ; L_{w}\right)$ under the sup norm.

Proof. The function $\vartheta^{(\infty)}(r, f, x)$ satisfies $(20)$ for $\underline{w}=(2(\kappa+r), 0)$ and $\chi=\varepsilon_{\rho}$ simply from the definition of $\omega_{t}$ and because if $\rho_{\mathbb{A}}(t)=d_{0} g_{\infty} \hat{r}$ under $(21)$, then $\rho_{\mathbb{A}}(t \lambda z u)=\left(d_{0} \lambda\right)\left(g_{\infty} z\right)(\hat{r} u)$ and $\rho_{\infty}(z)$ fixes $\tau$.

If $I=[t] \in \mathcal{I}_{\mathfrak{n}_{\varepsilon_{\rho}}}$ with $t_{v}=1$ for all $v \mid \infty \mathfrak{n}_{\varepsilon_{\rho}}$, write $t=t_{s} \lambda z u$ for some $\lambda \in K^{\times}, z \in \mathbb{C}^{\times}, u \in \widehat{\mathcal{O}}_{K, c}^{\times}$and $t_{s} p$-normalized. Then $\lambda z=1$, so that

$$
\vartheta(r, f, x)(I)=\vartheta^{(\infty)}(r, f, \rho)\left(t_{s} \lambda z u\right)=\lambda^{2(\kappa+r)} \theta_{w}^{(r)}\left(f, x^{s}\right)
$$

for some $X_{1}^{\Delta}(N) \ni x^{s} \mapsto x_{0}^{s}$ and theorem 2.9 applies. Finally, if the ideal $I$ is also prime to $p$ the right hand side in $(23)$ is in $\mathcal{O}_{(w)}$, again by theorem 2.9, because $\lambda$ is a $p$-unit.

With a slight abuse of notation, denote $L_{w}^{\prime}$ the completion of $L_{w}\left(\Omega_{p}\right)$ with ring of integers $\mathcal{O}_{w}^{\prime}$.

Proposition 2.16. There is a unique $\mathfrak{S}\left(\mathfrak{n}_{\varepsilon_{\rho}}^{*} ; L_{w}^{\prime}\right)$-valued measure $\mu_{f, \rho}$ on $\mathbb{Z}_{p}$ such that

$$
m_{r}\left(\mu_{f, \rho}\right)=\int_{\mathbb{Z}_{p}} t^{r} d \mu_{f, \rho}(t)=\Omega_{p}^{2(\kappa+r)} \vartheta^{(p)}(r, f, x), \quad \text { for all } r \geq 0 .
$$


Proof. Formula (23) together with the fact that $\lambda \in \mathbb{Z}_{p}^{\times}$because $p$ splits in $K$ says that on the dense subset $\mathcal{I}_{p \mathfrak{n}_{\varepsilon_{\rho}}}$ the value $\Omega_{p}^{2(\kappa+r)} \vartheta(r, f, x)(I)=\lambda^{2 \kappa} m_{r}\left(\mu_{f, x^{s}}\right)$ is the $r$-th moment of a $L_{w}^{\prime}$-valued measure $\mu_{I}$ on $\mathbb{Z}_{p}$. It is clear by continuity and the existence of the $p$-adic avatars $\vartheta^{(p)}(r, f, x)$ that for any continuous $\mathcal{O}_{w^{-}}^{\prime}$ valued function $\varphi$ on $\mathbb{Z}_{p}$ the function $I \mapsto \int_{\mathbb{Z}_{p}} \varphi(t) d \mu_{I}(t)$ extends uniquely to $\mathfrak{C}_{\mathfrak{n}_{\varepsilon_{\rho}}^{*}}$ to a continuous $\mathcal{O}_{w}^{\prime}$-valued function.

Corollary 2.17. $m_{r}\left(\mu_{f, \rho}^{\times}\right)=\Omega_{p}^{2(\kappa+r)} \vartheta^{(p)}(r,(1-V U) f, x)$.

Proof. This is a direct application of theorem 2.11.

Remark 2.18. In the next section the functions $\vartheta(r, f, x)$, or any of their avatars, will be paired against characters. Proposition 2.12 suggests to consider $\mathfrak{S}\left(\mathfrak{n}_{\varepsilon_{\rho}}^{*} ; F_{w}\right)$-valued measures with moments characters of weight $(-2(\kappa+r), 0)$. A basic way to obtain such measures is as follows. Let $\chi_{0} \in H_{(-2 \kappa, 0)}\left(\mathfrak{n} ; E_{0}\right)$ and $\chi \in H_{(2,0)}(\mathfrak{n} ; E)$ be characters such that $p$ splits completely in $E$ and let $\chi_{r}=\chi_{0} \chi^{r}$ for all $r \geq 0$. Let $F$ be a $p$-adic field containing both $E_{0}$ and $E$. Then there exists a $\mathfrak{S}(\mathfrak{n} ; F)$-valued meaure $\mu_{\chi_{0}, \chi}$ on $\mathbb{Z}_{p}$ such that

$$
m_{r}\left(\mu_{\chi_{0}, \chi}\right)=\int_{\mathbb{Z}_{p}} t^{r} d \mu_{\chi_{0}, \chi}=\chi_{r}^{(p)}, \quad \text { for all } r \geq 0 .
$$

Indeed, evaluation at every $s \in \mathfrak{C}_{\mathfrak{n}}$ defines a scalar valued $p$-adic distribution $\mu_{\chi_{0}, \chi}(s)$ whose associated power series is

$$
\Phi_{\mu_{\chi_{0}, \chi}(s)}(Z)=\sum_{r=0}^{\infty} \frac{\chi_{r}^{(p)}(s)}{r !} Z^{r}=\chi_{0}^{(p)}(s)(1+T)^{\chi^{(p)}(s)} \quad\left(T=e^{Z}-1\right),
$$

which has bounded coefficients in the variable $T$ since our assumption on $p$ implies that $\chi^{(p)}$ takes values in $\mathbb{Z}_{p}^{\times}$(in fact, $\mu_{\chi_{0}, \chi}(s)$ is simply a scalar multiple of the Dirac measure at $\left.\chi(s)^{(p)}\right)$. As in the previous proof, this entails that $\mu_{\chi_{0}, \chi}$ is a measure which in fact is supported on $\mathbb{Z}_{p}^{\times}$, i.e $\mu_{\chi_{0}, \chi}^{\times}=\mu_{\chi_{0}, \chi}$.

\section{Interpolation}

\subsection{Some periods computations}

For any $f^{\Delta} \in M_{0, k, \infty}^{\Delta}(N, \varepsilon)$ let $F_{f} \Delta \in L^{2}\left(D_{\mathbb{Q}}^{\times} \backslash D_{\mathbb{A}}^{\times}, \varepsilon_{\mathbb{A}}\right)$ the usual adelic lift of $f^{\Delta}$, namely $F_{f} \Delta(d)=f^{\Delta}\left(g_{\infty} \cdot i\right) j\left(g_{\infty}, i\right)^{-k} \operatorname{det}\left(g_{\infty}\right)^{k / 2} \varepsilon_{\mathbb{A}}(\hat{r})$ if $d=d_{\mathbb{Q}} g_{\infty} \hat{r}$ under (21). The Lie algebra $\mathfrak{g}=\mathfrak{g l}_{2} \simeq \operatorname{Lie}\left(D_{\infty}^{\times}\right)$acts on the $\mathbb{C}$-valued $\mathcal{C}^{\infty}$ functions on $D_{\mathbb{A}}^{\times}$by $A * \varphi(d)=\left.\frac{d}{d t} \varphi\left(d e^{t A}\right)\right|_{t=0}$ and the action extends to the complexified universal enveloping algebra $\mathfrak{A}(\mathfrak{g})_{\mathbb{C}}$. Let $I=\left(\begin{array}{ll}1 & 0 \\ 0 & 1\end{array}\right), H=\left(\begin{array}{cc}0 & -i \\ i & 0\end{array}\right), X^{ \pm}=\frac{1}{2}\left(\begin{array}{cc}1 & \pm i \\ \pm i & -1\end{array}\right)$ be the standard eigenbasis of $\mathfrak{g}_{\mathbb{C}}$ for the adjoint action of the maximal compact subgroup $\mathrm{SO}(2)=\left\{r_{\theta}=\left(\begin{array}{cc}\cos \theta & -\sin \theta \\ \sin \theta & \cos \theta\end{array}\right)\right.$ such that $\left.\theta \in \mathbb{R}\right\}$. Since $\operatorname{Ad}\left(r_{\theta}\right) X^{ \pm}=$ $e^{\mp 2 i \theta} X^{ \pm}$, the function $X^{ \pm} * \phi_{F}$ is a lift from $M_{0, k \pm 2, \infty}^{\Delta}(N, \varepsilon)$. A standard 
computation (e.g. $[3, \S \S 2.1-2])$ links the Lie action to the archimedean Maass operators of $\S 1.3$, namely $X^{+} * F_{f} \Delta=-4 \pi \phi_{\delta_{2 \kappa} f^{\Delta}}$. For $r \geq 0$ let

$$
F_{f \Delta}^{(r)}=\left(-\frac{1}{4 \pi} X^{+}\right)^{r} * F_{f \Delta}=F_{\delta_{2 \kappa}^{(r)} f^{\Delta}} .
$$

Definition 3.1. Let $f^{\Delta} \in M_{0,2 \kappa}^{\Delta}(N, \varepsilon), \rho \in \operatorname{Hom}^{\sharp}(K, D)$ of conductor $c$ with fixed point $\tau \in \mathrm{CM}_{\Delta, K}$ and $\xi$ a character of $K_{\mathbb{A}}^{\times}$trivial on $K^{\times} \mathbb{R}^{\times}$. For each $r \geq 0$ let

$$
J_{r}(f, \xi, \tau)=\int_{K_{\mathbb{A}}^{\times} / K^{\times} \mathbb{R}^{\times}} F_{f^{\Delta}}^{(r)}\left(\rho(t) d_{\infty}^{\tau}\right) \xi(t) d t
$$

where $d t$ is the Haar measure on $K_{\mathbb{A}}^{\times}$whose archimedean component is normalized so that $\operatorname{vol}\left(\mathbb{C}^{\times} / \mathbb{R}^{\times}\right)=\pi$ and such that the local groups of units have volume 1 .

Note that $J_{r}(f, \xi, \tau)=0$ when $\chi \xi_{\mid U} \neq 1$ where $U$ is the largest subgroup of finite ideles in $K_{A}^{\times}$such that $F_{f^{\Delta}}^{(r)}(\rho(t))$ is right $\chi$-invariant for some character $\chi$ of $U$. We shall implicitly assume that $\hat{\mathcal{O}}_{K, c}^{\times}=U$ The following proposition relates $J_{r}(f, \xi, \tau)$ to the pairing (19).

Proposition 3.2. In the situation of definition 3.1 assume $\xi \in H_{\underline{w}}^{(\infty)}\left(c, \varepsilon_{\rho}^{-1}\right)$ with $\underline{w}=(w,-w)$ and that $\left(\mathcal{O}_{K, c}^{\times}\right)^{2(\kappa+r)}=1$. Then

$$
J_{r}(f, \xi, \tau)=2 \pi \frac{\operatorname{vol}\left(\hat{\mathcal{O}}_{K, c}^{\times}\right) h_{c}^{\sharp}}{\left|\mathcal{O}_{K, c}^{\times}\right|} \frac{1}{\left(\operatorname{Im}(\tau) \Omega_{\infty}(\tau)^{2}\right)^{w}}\left\langle\vartheta^{(\infty)}(r, f, x), \xi\left\|N_{K / \mathbb{Q}}\right\|^{-w}\right\rangle_{\infty}
$$

Proof. The integrand is right $\hat{\mathcal{O}}_{K, c^{\times}}^{\times}$-invariant. We can write $K_{A}^{\times} / K^{\times} \mathbb{R}^{\times} \hat{\mathcal{O}}_{K, c}^{\times}=$ $\bigcup_{s \in \mathbf{C}_{c}^{\sharp}} t_{s} \mathbb{C}^{\times} / \mathbb{R}^{\times} \mathcal{O}_{K, c}^{\times}$as a disjoint union for some set of representatives

$$
J_{r}(f, \xi, \tau)=\operatorname{vol}\left(\hat{\mathcal{O}}_{K, c}^{\times}\right) \sum_{s \in \mathbf{C}_{c}^{\sharp}} \xi\left(t_{s}\right) \int_{\mathbb{C}^{\times} / \mathbb{R}^{\times} \mathcal{O}_{K, c}^{\times}} F_{f^{\Delta}}^{(r)}\left(\rho\left(t_{s} z\right) d_{\infty}^{\tau}\right) \xi_{\infty}(z) d^{\times} z .
$$

To compute each summand write $z=r e^{i \theta}$ and $\rho_{\infty}(z)=r d_{\infty}^{\tau} r_{\theta}\left(d_{\infty}^{\tau}\right)^{-1}$ so that

$$
\int_{\mathbb{C}^{\times} / R^{\times} \mathcal{O}_{K, c}^{\times}} F_{f^{\Delta}}^{(r)}\left(\rho\left(t_{s} z\right) d_{\infty}^{\tau}\right) \xi_{\infty}(z) d^{\times} z=\frac{F_{f \Delta}^{(r)}\left(\rho\left(t_{s}\right) d_{\infty}^{\tau}\right)}{\left|\mathcal{O}_{K, c}^{\times}\right|} \int_{0}^{2 \pi} e^{-2 i(\kappa+r+w) \theta} d \theta .
$$

Thus $J_{r}(f, \xi, \tau)=0$ if $w \neq-\kappa-r$ proving the formula in this case since also the scalar product vanishes due to the incompatibility of weights. To finish the proof, write $t_{s}=d_{s} g_{s} u_{s}$ under (21). Then, under the hypotheses that $t_{s}$ is normalized and $\left(\mathcal{O}_{K, c}^{\times}\right)^{2 \kappa+r}=1$, the first identity in (22) implies that

$$
\xi\left(t_{s}\right) F_{f^{\Delta}}^{(r)}\left(t_{s} d_{\infty}^{\tau}\right)=\operatorname{Im}(\tau)^{\kappa+r} \Omega_{\infty}(\tau)^{2(\kappa+r)} \vartheta^{(\infty)}\left(r, f^{\Delta}, x\right) \xi\left(t_{s}\right)\left\|\mathrm{N}_{K / \mathbb{Q}}\left(t_{s}\right)\right\|^{\kappa+r}
$$

since $\operatorname{det}\left(g_{s}\right)=\left\|\mathrm{N}_{K / \mathbb{Q}}\left(t_{s}\right)\right\|$. 
We shall henceforth assume that $\xi$ satisfies the hypothesis in proposition 3.2. Denote $\mathfrak{p}^{s}$ the conductor of the local component $\xi_{\mathfrak{p}}$. For the next proposition assume also that the CM point $\tau$ represents a point $x \in \mathcal{X}_{\mathrm{ar}}^{\Delta}\left(M p^{\nu}\right)\left(\mathcal{O}_{v_{\nu}}\right)$ in a $p$-ordinary split arithmetic test object, with $(M, p)=1$.

Proposition 3.3. Suppose that $(p, c)=1$. Then, there exists a uniformizer $\varpi_{\mathfrak{p}}$ of $\mathfrak{p} \mathcal{O}_{\mathfrak{p}}$, well-defined up to $\left(1+\mathfrak{p}^{s}\right)$-association such that

$$
J_{r}\left(V f^{\Delta}, \xi, \tau\right)=p^{-2 \kappa} \xi_{\mathfrak{p}}\left(\varpi_{\mathfrak{p}}\right) J_{r}\left(f^{\Delta}, \xi, \tau\right) .
$$

Proof. Over $\mathbb{C}$, where modular forms can be regarded as homogeneous functions of lattices and level structures, $V f^{\Delta}(P, \Lambda)=p^{-2 \kappa} f^{\Delta}\left(P^{\prime}, \Lambda^{\prime}\right)$ where $P$ is a point of period $M p^{\nu+1}$ in $A=\mathbb{C}^{\varepsilon} / \Lambda, \Lambda^{\prime}$ is the lattice $(\mathcal{R}$-module when $\Delta>1$ ) generated by $\Lambda$ and $\bar{P}=M p^{\nu} P$, and $P^{\prime}$ the image of $P$ in $A^{\prime}=\mathbb{C}^{\varepsilon} / \Lambda^{\prime}$ under the natural quotient map (clearly a point of period $M p^{\nu}$ there).

In our situation of arithmetic structures, the group ( $\mathcal{R}$-module) generated by $\bar{P}$ in $A_{\tau}=\mathbb{C}^{\varepsilon} / \Lambda_{\tau}$ is the base change to $\mathbb{C}$ of $\eta_{p}\left(\mu_{p}^{\varepsilon}\right)$. Thus, if $\sigma_{\mathfrak{p}}=\left(\mathfrak{p}, H_{c} / K\right)$ is the Frobenius at $\mathfrak{p}, \mathbb{C}^{\varepsilon} / \Lambda_{\tau}^{\prime}=A_{x}^{\sigma_{\mathfrak{p}}}(\mathbb{C})$ and the natural quotient map induced by the inclusion $\Lambda_{\tau} \subset \Lambda_{\tau}^{\prime}$ is a lifting to $\mathbb{C}$ of the Frobenius map between the varieties reduced modp.

Since $(p, c)=1$, we can write $\sigma_{\mathfrak{p}}=\left[\widetilde{\varpi}_{\mathfrak{p}}, K\right]_{\mid H_{c}}$ where $\widetilde{\varpi}_{\mathfrak{p}} \in K_{\mathbb{A}}^{\times}$has component a uniformizer $\varpi_{\mathfrak{p}}$ at $\mathfrak{p}$ and 1 elsewhere. Again by Shimura reciprocity we may take $\Lambda_{\tau}^{\prime}=\widetilde{\varpi}_{\mathfrak{p}}^{-1} \Lambda_{\tau}$ so that $\bar{P}$ is a generator (as a group or as $\mathcal{R}$-module) of $\widetilde{\varpi}_{\mathfrak{p}}^{-1} \Lambda_{\tau} / \Lambda_{\tau}$ and we also know that the action of $\left[\widetilde{\varpi}_{\mathfrak{p}}, K\right]$ on division points is given by the "multiplication by $\widetilde{\varpi}_{\mathfrak{p}}^{-1}$ "map

$$
\Lambda_{\tau} \otimes \mathbb{Q} / \Lambda_{\tau} \stackrel{\widetilde{\varpi}_{\mathfrak{p}}^{-1}}{\longrightarrow} \Lambda_{\tau} \otimes \mathbb{Q} / \widetilde{\varpi}_{\mathfrak{p}}^{-1} \Lambda_{\tau} \hookrightarrow A_{x}^{\sigma_{p}}(\mathbb{C}),
$$

which is actually defined locally. Forgetting one $p$-level in the structure simply amounts to moving from $(P, \Lambda)$ to $(p P, \Lambda)$ and so choose $\varpi_{\mathfrak{p}}$ so that $p P \mapsto P^{\prime}$ under (25). This choice of local uniformizer is well-defined only up to a local unit in the stabilizer of $p P$ (i.e. the kernel of the $p$-orientations, $\S 1.1$ ), and for our purposes up to a local unit in the kernel of $\tilde{\xi}_{\mathfrak{p}}$ as this coincides with the kernel of the local component of the character induced by the nebentypus. It follows immediately from the commutativity of the relevant Galois groups that the same uniformizer $\varpi_{\mathfrak{p}}$ works also replacing $x$ with any other point in in the fiber of $\operatorname{Im}\left(\bar{\rho}_{\mathbb{A}}\right) \subset X_{0}^{\Delta}\left(M p^{\nu}\right)$, i.e. on this fiber the modular definition of $p^{2 \kappa} V$ corresponds to translation by $\widetilde{\varpi}_{\mathfrak{p}}^{-1} \in K_{\mathbb{A}}^{\times}$. Thus

$$
F_{V f^{\Delta}}^{(r)}\left(\rho(t) d_{\infty}^{\tau}\right)=p^{-2 \kappa} F_{f^{\Delta}}^{(r)}\left(\rho\left(t \widetilde{\varpi}_{\mathfrak{p}}^{-1}\right) d_{\infty}^{\tau}\right)
$$

and the formula now follows readily from an integration by substitution.

Corollary 3.4. Suppose in addition that $f^{\Delta}$ is a newform with $T_{p}\left(f^{\Delta}\right)=a_{p} f^{\Delta}$. Then

$$
J_{r}\left((1-V U) f^{\Delta}, \xi, \tau\right)=\left(1-a_{p} \xi_{\mathfrak{p}}\left(\varpi_{\mathfrak{p}}\right) p^{-2 \kappa}+\varepsilon(p) \xi_{\mathfrak{p}}\left(\varpi_{\mathfrak{p}}\right)^{2} p^{-2 \kappa-1}\right) J_{r}\left(f^{\Delta}, \xi, \tau\right) .
$$

Proof. This follows immediately form the previous proposition recalling that $T_{p}=U+\varepsilon(p) p^{2 \kappa-1} V$. 


\subsection{Central $L$-values}

Let $\operatorname{GO}(Q)$ denote the algebraic group of similitudes of the quadratic space $Q$. When $Q=D$ with respect to the reduced norm $\nu$ the group $\operatorname{GO}(D)$ is completely described by the short exact sequence

$$
1 \longrightarrow \mathbb{G}_{m} \longrightarrow D^{\times} \times D^{\times} \stackrel{\varrho}{\longrightarrow} \mathrm{GO}(D)^{\circ} \longrightarrow 1, \quad \varrho(x, y)(d)=x d y^{-1},
$$

together with $\operatorname{GO}(D)=\operatorname{GO}(D)^{o} \ltimes\langle\mathbf{t}\rangle$, where $\mathbf{t}(d)=\bar{d}$, e.g. [9, §1.1], [10, §7]. The decomposition $D=K \oplus K u$ associated to $\rho \in \operatorname{Hom}^{\sharp}(K, D)$ is orthogonal and the norm $\nu$ restricts to $\mathrm{N}_{K / \mathbb{Q}}$ and $-u^{2} \mathrm{~N}_{K / \mathbb{Q}}$ on $K$ and $K u$ respectively. Let $T=\mathrm{G}(\mathrm{O}(K) \times \mathrm{O}(K u))<\mathrm{GO}(D)$ be the subgroup preserving the splitting. Left multiplication gives isomorphisms $\mathrm{GO}(K)^{\circ} \simeq R_{K / \mathbb{Q}} \mathbb{G}_{m, K} \simeq \mathrm{GO}(K u)^{\circ}$ and there is an identification

$$
T^{\circ}=\left\{\left(k_{1}, k_{2}\right) \in R_{K / \mathbb{Q}} \mathbb{G}_{m, K}^{2} \text { such that } \mathrm{N}_{K / \mathbb{Q}}\left(k_{1} k_{2}^{-1}\right)=1\right\} .
$$

Also, there is a commutative diagram

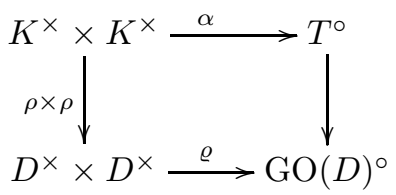

where $\alpha\left(k_{1}, k_{2}\right)=\left(k_{1} k_{2}^{-1}, k_{1} \bar{k}_{2}^{-1}\right)$.

Let $\mathcal{S}(D)_{\mathbb{A}}=\bigotimes_{\ell<\infty} \mathcal{S}(D)_{\ell}$ denote the space of adelic Schwartz-Bruhat functions whose archimedean component consists of classical Schwartz functions on $D_{\infty}$ which are $K_{\infty}^{1} \times K_{\infty}^{1}$-finite under the action of $\varrho$, where $K_{\infty}^{1}$ is the maximal compact subgroup of $\rho(K \otimes \mathbb{R}) \subset D_{\infty}^{\times} \simeq \mathrm{GL}_{2}(\mathbb{R})$. The space $\mathcal{S}(D)_{\mathbb{A}}$ supports the representation $\tilde{r}_{\psi}$ of the adelic points of the group

$$
R(D)=\left\{(g, h) \in \mathrm{GL}_{2} \times \mathrm{GO}(D) \mid \operatorname{det}(g)=\nu_{0}(h)\right\} \simeq \mathrm{SL}_{2} \ltimes \mathrm{GO}(D)
$$

defined to be

$$
\tilde{r}_{\psi}(g, h) \varphi=r_{\psi}\left(g\left(\begin{array}{c}
{ }^{1} \nu_{0}(h) \\
{ }^{\prime}
\end{array}\right)^{-1}\right) L(h) \varphi=L(h) r_{\psi}\left(\left({ }^{1} \nu_{0}(h)\right)^{-1} g\right) \varphi
$$

where $r_{\psi}$ is the Weil representation of $S L_{2}(\mathbb{A})$ and $L$ the unitary representation of $\operatorname{GO}(D)(\mathbb{A})$ given by $L(h) \varphi(x)=\left\|\nu_{o}(h)\right\|_{\mathbb{A}}^{-1} \varphi\left(h^{-1} x\right)$ for all $x \in D_{\mathbb{A}},[11, \S 5]$. The group $R(D)$ is naturally a subgroup of $\operatorname{Sp}(\mathbb{P} \otimes D)$, with $\mathbb{P}$ the hyperbolic plane, via $(g, h) x \otimes y=g x \otimes h^{-1} y$. The groups $\left(\mathrm{SL}_{2}, \mathrm{O}(D)\right)$ form a dual reductive pair in $\operatorname{Sp}(\mathbb{P} \otimes D)$ and the extended Weil representation $\tilde{r}_{\psi}$ allows to realize the theta correspondence between the similitude groups by integrating the theta kernel

$$
\vartheta(g, h ; \varphi)=\sum_{d \in D} \tilde{r}_{\psi}(g, h) \varphi(d)
$$


against automorphic forms. Namely, if $F$ is a cuspidal automorphic form on $\mathrm{GL}_{2}$ the function defined as

$$
\theta_{\varphi}(F)(h)=\int_{\mathrm{SL}_{2}(\mathbb{Q}) \backslash \mathrm{SL}_{2}(\mathbb{A})} \vartheta\left(g^{\prime} g, h ; \varphi\right) F\left(g^{\prime} g\right) d g^{\prime}
$$

for any choice of $g \in \mathrm{GL}_{2}(\mathbb{A})$ such that $(g, h) \in R(D)_{\mathbb{A}}$ is an automorphic form on $\operatorname{GO}(D)$. Denote $\widetilde{\theta}_{\varphi}(F)$ the automorphic form on $D_{\mathbb{A}}^{\times} \times D_{\mathbb{A}}^{\times}$obtained from $\theta_{\varphi}(F)$ by pull-back along the map $\varrho$ in (26). For later use, note that a straightforward substitution yields the formula

$$
\theta_{\varphi}(F)\left(h h_{1}\right)=\theta_{\tilde{r}_{\psi}\left(g_{1}, h_{1}\right) \varphi}\left(\pi\left(g_{1}\right) F\right)
$$

valid for all $\left(g_{1}, h_{1}\right) \in R(D)$. Likewise, if $G$ is an automorphic form on $\operatorname{GO}(D)$ the function defined on the subgroup $\mathrm{GL}_{2}(\mathbb{A})^{+}$consisting of the $g \in \mathrm{GL}_{2}(\mathbb{A})$ such that $(g, h) \in R(D)_{\mathbb{A}}$ for some $h \in \mathrm{GO}(V)(\mathbb{A})$ as

$$
\theta_{\varphi}^{t}(G)(g)=\int_{\mathrm{O}(V)(\mathbb{Q}) \backslash \mathrm{O}(V)(\mathbb{A})} \vartheta\left(g, h^{\prime} h ; \varphi\right) G\left(h^{\prime} h\right) d h^{\prime}
$$

and extended first to $\mathrm{GL}_{2}(\mathbb{Q}) \mathrm{GL}_{2}(\mathbb{A})^{+}$by left $\mathrm{GL}_{2}(\mathbb{Q})$-invariance and set equal to 0 outside is an automorphic form on $\mathrm{GL}_{2}(\mathbb{A})$.

Let $f^{\Delta} \in M_{0,2 \kappa}^{\Delta}(N, \varepsilon)$ be a newform with associated automorphic representation $\pi^{D}$ of $D_{\mathbb{A}}^{\times}$with central character $\varepsilon$. Denote $\pi=\operatorname{JL}\left(\pi^{D}\right)$ the automorphic representation of $\mathrm{GL}_{2}(\mathbb{A})$ corresponding to $\pi^{D}$ under the Jacquet-Langlands correspondence. Let $\widetilde{\Theta}(\pi)=\left\{\widetilde{\theta}_{\varphi}(F)\right\}_{F \in \pi, \varphi \in \mathcal{S}(D)_{\mathbb{A}}}$ be the space of automorphic forms on $D^{\times} \times D^{\times}$which are pull-backs of theta lifts (28) from $\pi$. As pointed out in $[9,10]$ the work of Shimizu [28] yields

$$
\widetilde{\Theta}(\pi)=\pi^{D} \otimes \check{\pi}^{D},
$$

where $\check{\pi}^{D} \simeq \pi^{D} \otimes\left(\varepsilon^{-1} \circ \nu\right)$ is the contragredient representation of $\pi^{D}$. If $F$ and $\varphi$ are chosen so that $\widetilde{\theta}_{\varphi}(F)=\pi\left(d_{\infty}^{\tau}\right) F_{f^{\Delta}}^{(r)} \otimes \pi\left(d_{\infty}^{\tau}\right) F_{f^{\Delta}}^{(r)} \cdot\left(\varepsilon^{-1} \circ \nu\right)$ then

$$
\left.L_{\underline{\xi}} \widetilde{\theta}_{\varphi}(F)\right):=\int_{\left(K^{\times} \mathbb{R} \times \backslash K_{\mathbb{A}}^{\times}\right)^{2}} \tilde{\theta}_{\varphi}(F) \underline{\xi}(t) d t=J_{r}\left(f^{\Delta}, \xi, \tau\right)^{2},
$$

where the pair $\underline{\xi}=(\xi, \xi(\varepsilon \circ N))$ can be thought of as a character of $K_{\mathbb{A}}^{\times} \times$ $K_{\mathbb{A}}^{\times}$. On the other hand, Harris and Kudla observe that for all $F$ and $\varphi$ the integral $\left.L_{\underline{\xi}} \widetilde{\theta}_{\varphi}(F)\right)$ can be read, via the map $\alpha$ in $(27)$ as the Petersson scalar product of two automorphic forms on the group $T$, namely $L_{\underline{\xi}}\left(\widetilde{\theta}_{\varphi}(F)\right)=$ $\int_{T(\mathbb{Q}) T(\mathbb{R}) \backslash T(\mathbb{A})} \widetilde{\theta}_{\varphi}(F)(a, b) \xi(b) d^{\times} a d^{\times} b$, where $\alpha(t)=(a, b)$. Thus, the seesaw identity $[24,1.14]$ associated with the seesaw dual pair

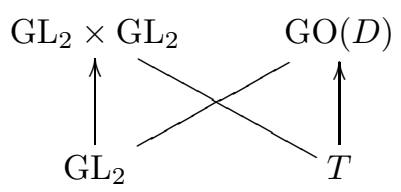


identifies, up to a renormalization of the Haar measures, the value $L_{\underline{\xi}}\left(\widetilde{\theta}_{\varphi}(F)\right)$ with a scalar product on $\mathrm{GL}_{2}$,

$$
L_{\underline{\xi}}\left(\widetilde{\theta}_{\varphi}(F)\right)=\int_{\mathrm{GL}_{2}(\mathbb{Q}) \mathbb{A}^{\times} \backslash \mathrm{GL}_{2}(\mathbb{A})} F(g) \theta_{\varphi}^{t}(1, \xi)(g, g) d g,
$$

where $\theta_{\varphi}^{t}(1, \xi)$ denotes the theta lift from $T$ to $\mathrm{GL}_{2} \times \mathrm{GL}_{2}$ which is defined in a fashion similar to (28). If $\varphi=\varphi_{1} \otimes \varphi_{2}$ is split under a decomposition $D(\mathbb{A})=\left(K \oplus K^{\perp}\right) \otimes \mathbb{A}$ induced from an embedding $\rho: K \hookrightarrow D$ then $\theta_{\varphi}^{t}(1, \xi)$ is a product of two separate lifts: $\theta_{\varphi}^{t}(1, \xi)(g, g)=E(0, \Phi, g) \theta_{\varphi_{2}}(\xi)(g)$ where

- $\theta_{\varphi_{2}}(\xi)(g)=\int_{K^{(1)} \backslash K_{\mathbb{A}}^{(1)}} \vartheta\left(g, k k^{\prime} ; \varphi_{2}\right) \xi\left(k k^{\prime}\right) d k^{\prime}$ (for all $g \in \mathrm{GL}_{2}$ and $k \in K_{\mathbb{A}}^{\times}$ with $N k=\operatorname{det} g)$ is the binary form in the automorphic representation $\pi(\xi)$ of $\mathrm{GL}_{2}$ attached to $\xi$;

- $E(0, \Phi, g)$ is the value at $s=0$ of the holomorphic Eisenstein series attached to the unique flat section $([3, \S 3.7])$ extending the function $\Phi(g)=$ $r_{\psi}(g, k) \varphi_{1}(0)$ where $k \in K_{\mathbb{A}}^{\times}$is such that $N(k)=\operatorname{det}(g)$ and $r_{\psi}$ denotes here the extended adelic Weil representation attached to $K$ as a normed space (Siegel-Weil formula).

This expression yields a relation between the right hand side of (33) and the value at the centre of symmetry of a Rankin-Selberg convolution integral. Furthermore, if each Schwartz-Bruhat function is primitive, i.e. decomposes as a product of local factors $\varphi_{i}=\bigotimes_{\ell \leq \infty} \varphi_{\ell, i}(i=1,2)$, and if the Whittaker function $W_{F}$ of $F$ decomposes as a product of local Whittaker functions, the Rankin-Selberg integral admits an Euler decomposition [15, §19]. Precisely (see also $[27, \S 3.2])$

$$
L_{\underline{\xi}}\left(\widetilde{\theta}_{\varphi}(F)\right)=\left.\prod_{\ell \leq \infty} L_{\ell}\left(\varphi_{\ell}, \xi_{\ell}, s\right)\right|_{s=1 / 2},
$$

where $L_{\ell}\left(\varphi_{\ell}, \xi_{\ell}, s\right)$ is the analytic continuation of

$$
\begin{array}{r}
\int_{K_{\ell}} \int_{\mathbb{Q}_{\ell}^{\times}} W_{F, \ell}^{\psi_{\ell}}\left(\left(\begin{array}{cc}
a & 0 \\
0 & 1
\end{array}\right) k\right) W_{\theta_{\varphi_{2}, \ell}}^{\psi_{\ell}}\left(\left(\begin{array}{cc}
-a & 0 \\
0 & 1
\end{array}\right) k\right) . \\
\Phi_{\varphi_{1}, \ell}^{s}\left(\left(\begin{array}{cc}
a & 0 \\
0 & 1
\end{array}\right) k\right)|a|^{-1} d^{\times} a d k_{\ell}
\end{array}
$$

with the local measures normalized so that $K_{\infty}=\mathrm{SO}_{2}(\mathbb{R})$ has volume $2 \pi$ and $K_{\ell}=\mathrm{GL}_{2}\left(\mathbb{Z}_{\ell}\right)$ has volume 1 for finite $\ell, W_{\theta_{\varphi_{2}}}$ is the Whittaker function and $\Phi^{s}(g)=\|a\|^{s-\frac{1}{2}} \Phi(g)$ under the $N A K$-decomposition $g=n a k$ with $\left\|\left(\begin{array}{c}a \\ b\end{array}\right)\right\|=$ $|a / b|$. The local term (34) does not vanish and for almost all $\ell$ can be recognized as a quotient of Euler factors. Thus one obtains, as in [9, 2.3.11] and [10, §14], a version of Waldspurger's result [30]. Namely,

$$
L_{\underline{\xi}}\left(\tilde{\theta}_{\varphi}(F)\right)=\left.\Lambda(\varphi, \xi, s) L\left(\pi_{K} \otimes \xi, s\right) L\left(\eta_{K}, 2 s\right)^{-1}\right|_{s=1 / 2},
$$


where $\Lambda(\varphi, \xi, s)$ is a finite product of local integrals, $\pi_{K}$ is the base change to $K$ of the automorphic representation $\pi$ and $L\left(\eta_{K}, 2 s\right)$ is the Dirichlet $L$-function attached to $\eta_{K}$, the quadratic character associated to $K$. Note that the single local terms (34) do depend on the choice of $u \in K^{\perp}$.

\subsection{Making the theta correspondence explicit}

Let us fix complex coordinates in $D_{\infty}$ as follows. The normalized standard embedding $\rho^{\text {st }}: \mathbb{Q}(\sqrt{-1}) \hookrightarrow \mathrm{M}_{2}(\mathbb{Q})$ with fixed point $i \in \mathfrak{H}$ defines a splitting $\Phi_{\infty}\left(D_{\infty}\right)=\mathbb{C} \oplus \mathbb{C}^{\perp}$ with $\mathbb{C}=\mathbb{R}\left({ }^{1}{ }_{1}\right) \oplus \mathbb{R}\left({ }_{1}{ }^{-1}\right)$ and $\mathbb{C}^{\perp}=\mathbb{C}\left({ }^{1}{ }_{-1}\right)$. Thus, define standard complex coordinates $z_{i}=z_{i}^{\mathrm{st}}, i=1,2$ for $d \in D_{\infty}$ as

$$
\Phi_{\infty}(d)=z_{1}^{\mathrm{st}}(d)+z_{2}^{\mathrm{st}}(d)\left(\begin{array}{ll}
1 & \\
& -1
\end{array}\right) .
$$

We choose $\varphi^{\prime}=\bigotimes_{v} \varphi_{\ell}^{\prime}$ with local components given as follows.

- $\varphi_{\infty}^{\prime}\left(z_{1}, z_{2}\right)=\frac{1}{\pi} z_{2}^{2 \kappa} e^{-2 \pi\left(z_{1} \bar{z}_{1}+z_{2} \bar{z}_{2}\right)}$;

- $\varphi_{\ell}^{\prime}=\frac{1}{\operatorname{vol}\left(\mathcal{R}_{N, \ell}^{\times}\right)} \operatorname{ch}\left(\mathcal{R}_{N, \ell}\right)$ for all finite $\ell$ such that $\varepsilon_{\ell}$ is unramified;

- for all $\ell \mid N$ such that $\varepsilon_{\ell}$ is ramified and of conductor $\ell^{s}$ let $\varphi_{\ell}^{\prime}$ be the function supported on $S_{\ell}=\left(\begin{array}{ll}\ell^{s} \mathbb{Z}_{\ell} & \mathbb{Z}_{\ell}^{\times} \\ \ell^{s} \mathbb{Z}_{\ell} & \ell^{s} \mathbb{Z}_{\ell}\end{array}\right)$ such that

$$
\varphi_{\ell}^{\prime}\left(\begin{array}{cc}
\ell^{s} a & b \\
\ell^{s} c & \ell^{s} d
\end{array}\right)=\frac{1}{\operatorname{vol}\left(\mathcal{R}_{N, \ell}^{\times}\right)} \varepsilon_{\ell}^{-1}(b), \quad \forall\left(\begin{array}{cc}
\ell^{s} a & b \\
\ell^{s} c & \ell^{s} d
\end{array}\right) \in S_{\ell} .
$$

Note that for the archimedean prime and the finite primes for which $\varepsilon$ is unramified, our choice of test function $\varphi_{\ell}^{\prime}$ coincides with or is an immediate generalization of that of Prasanna [27] (itself an adaptation of [31]) where $N$ is assumed square-free and $\varepsilon$ unramified.

Let $F \in \pi$ be the lift of the holomorphic newform.

Proposition 3.5. $\widetilde{\theta}_{\varphi^{\prime}}(F)=F^{D} \otimes \check{F}^{D}=F^{D} \otimes F^{D}\left(\varepsilon^{-1} \circ \nu\right)$ where $F^{D}$ is an holomorphic eigenform in $\pi^{D}$ such that $\langle F, F\rangle=\left\langle F^{D}, F^{D}\right\rangle$.

Proof. Let $\left(d_{1}, d_{2}\right) \in D_{\mathbb{A}}^{\times} \times D_{\mathbb{A}}^{\times}$and $\left(r_{1}, r_{2}\right) \in \mathcal{R}_{N, \ell}^{\times} \times \mathcal{R}_{N, \ell}^{\times}$. It follows from (29) that $\widetilde{\theta}_{\varphi^{\prime}}(F)\left(d_{1} r_{1}, d_{2} r_{2}\right)=\widetilde{\theta}_{r_{\psi}(g, h) \varphi^{\prime}}(\pi(g) F)\left(d_{1}, d_{2}\right)$ for $h=\varrho\left(r_{1}, r_{2}\right)$ and any $g \in \mathrm{GL}_{2}(\mathbb{A})$ such that $\operatorname{det}(g)=\nu_{0}(h)$. Clearly we can take $g$ to have components 1 away from $\ell$ and, since $\nu_{o}(h)$ is a $\ell$-unit, $g_{\ell}=\left(\begin{array}{cc}{ }^{1} \nu_{o}(h)\end{array}\right)$. Thus, $\pi(g) F=F$ and $r_{\psi}(g, h) \varphi^{\prime}(d)=\varphi^{\prime}\left(r_{1}^{-1} d r_{2}\right)$. It is now completely straightforward to check that

$\widetilde{\theta}_{\varphi^{\prime}}(F)\left(d_{1} r_{1}, d_{2} r_{2}\right)=\varepsilon\left(\alpha_{1}\right) \varepsilon^{-1}\left(\delta_{2}\right) \widetilde{\theta}_{\varphi^{\prime}}(F)\left(d_{1}, d_{2}\right), \quad$ if $r_{i}=\left(\begin{array}{cc}\alpha_{i} & \beta_{i} \\ \gamma_{i} \ell^{n} & \delta_{i}\end{array}\right), i \in\{1,2\}$.

Given (31), Casselman's theory now applies to conclude that at every finite prime $\widetilde{\theta}_{\varphi^{\prime}}(F)$ is (proportional to) the tensor product of the newvectors. Holomorphicity in both variables is obtained from the choice of $\varphi_{\infty}^{\prime}$ as in [27], where 
the original argument in [31] is modified. The identity of Petersson norms can also be obtained as in [27].

Remark 3.6. Applying (29) again finally yields

$$
\widetilde{\theta}_{\varphi \sharp}(F)=\pi\left(d_{\infty}^{\tau}\right) F^{D} \otimes \pi\left(d_{\infty}^{\tau}\right) \check{F}^{D}
$$

where $\varphi^{\sharp}=r_{\psi}\left(1,\left(d_{\infty}^{\tau}, d_{\infty}^{\tau}\right)\right) \varphi^{\prime}$. Clearly $\varphi^{\sharp}$ differs from $\varphi^{\prime}$ only for the archimedean component. Since $\rho=d_{\infty}^{\tau} \rho^{\mathrm{st}}\left(d_{\infty}^{\tau}\right)^{-1}$, if $D_{\infty} \ni x=\rho\left(w_{1}\right)+\rho\left(w_{2}\right) u$ with $w_{1}, w_{2} \in K \otimes \mathbb{R}=\mathbb{C}$,

$$
\varphi_{\infty}^{\sharp}(x)=\frac{1}{\pi}\left(w_{2} \zeta_{u}\right)^{2 \kappa} e^{-2 \pi\left(w_{1} \bar{w}_{1}+|\nu(u)| w_{2} \bar{w}_{2}\right)}
$$

where $\zeta_{u} \in \mathbb{C}$ is such that $\left(d_{\infty}^{\tau}\right)^{-1} u d_{\infty}^{\tau}=\zeta_{u}\left(\begin{array}{ll}1 & \\ & -1\end{array}\right)$. In particular $\varphi_{\infty}^{\sharp}$ is split under the decomposition of $D_{\infty}$ induced by $\rho$.

The following lemma allows us to factor the local components $\varphi_{\ell}$ in terms of the splitting $D_{\ell}=(K \oplus K u) \otimes \mathbb{Q}_{\ell}$ induced by an embedding $\rho$.

Lemma 3.7. Let $L \subset D$ be an embedding of conductor $c$ with respect to $\mathcal{R}_{N}$ of the quadratic field $L$ with associated decomposition $D=L \oplus L u$. Let $\Lambda=L \cap \mathcal{R}_{N}$, $\Lambda^{\prime}=L u \cap \mathcal{R}_{N}$. Then

1. $D$ is split at $\ell$ if and only if $\left(u^{2}, \delta_{L}\right)_{\ell}=1$.

2. If $\ell$ is unramified in $L$ and $(\ell, c)=1$ then $\mathcal{R}_{N} \otimes \mathbb{Z}_{\ell}=\Lambda \otimes \mathbb{Z}_{\ell} \oplus \Lambda^{\prime} \otimes \mathbb{Z}_{\ell}$. Moreover $\Lambda^{\prime} \otimes \mathbb{Z}_{\ell}=\mathcal{I}_{\ell} u$ for some fractional ideal $\mathcal{I}_{\ell} \subset L \otimes \mathbb{Q}_{\ell}$ such that $\mathrm{N}\left(\mathcal{I}_{\ell}\right) \nu(u)=\ell^{v_{\ell}(N)}$.

Proof. The first statement follows from the characterization in terms of the Hilbert symbol of the representability of 0 by the norm form on $D \otimes \mathbb{Q}_{\ell}$. To prove the second statement observe first that if $\ell$ is unramified in $L$ and coprime with $c$ then $e_{\rho}\left(\mathcal{R}_{N}\right) \subset \mathcal{R}_{N, \ell}$ (this follows readily from the explicit expression of $e_{\rho}$ of $\S 1.2)$. Then the last part follows from a local discriminant computation.

The lemma yields immediately a decomposition $\varphi_{\ell}=\varphi_{\ell, 1} \otimes \varphi_{\ell, 2}$ for all finite $\ell$ such that $\left(\ell, c \delta_{K} N\right)=1$ and those $\ell \mid N$ for which $\varepsilon_{\ell}$ is unramified, where $\varphi_{\ell, 1}=\operatorname{ch}\left(\mathcal{O}_{K} \otimes \mathbb{Z}_{\ell}\right)$ and $\varphi_{\ell, 2}=\operatorname{ch}\left(\mathcal{I}_{\ell} u\right)$.

When $\ell \mid \delta_{K}$ the local component $\varphi_{\ell}$ does not decompose as a product. Write $K \otimes \mathbb{Q}_{\ell}=\mathbb{Q}_{p}\left(\varpi_{\ell}\right)$ with $\varpi_{\ell}^{2}=\lambda_{\ell} \ell$ with $\lambda_{\ell}$ a $\ell$-unit; also $\mathcal{O}_{K} \otimes \mathbb{Z}_{\ell}=\mathbb{Z}_{\ell}\left[\varpi_{\ell}\right]$. Following $[27, \S 3.4 .5]$ consider the standard local embedding $\rho_{\text {st }}: \mathbb{Q}_{p}\left(\varpi_{\ell}\right) \hookrightarrow$ $D \otimes \mathbb{Q}_{\ell} \simeq \mathrm{M}_{2}\left(\mathbb{Q}_{\ell}\right)$ given by $\rho_{\mathrm{st}}\left(\varpi_{\ell}\right)=\left(\begin{array}{cc}0 & 1 \\ \lambda_{\ell} & 0\end{array}\right)$. The embeddings $\rho$ and $\rho_{\text {st }}$ are conjugated by a matrix which can be proved to be in $\mathrm{GL}_{2}\left(\mathbb{Z}_{\ell}\right)$. Thus, if $\lambda \in K$ is such that $u$ is conjugated to $\rho_{\mathrm{st}}(\lambda)\left(\begin{array}{cc}1 & 0 \\ 0 & -1\end{array}\right)$ one eventually gets

$$
\varphi_{\ell}= \begin{cases}\sum_{j=0}^{\ell-1} \varphi_{\ell, 1, j} \otimes \varphi_{\ell, 2, j} & \text { if } \ell>2, \\ \sum_{i=0}^{1} \sum_{j=0}^{3} \varphi_{2,1,(i, j)} \otimes \varphi_{2,2,(i, j)} & \text { if } \ell=2\end{cases}
$$


with $\varphi_{\ell, 1, j}=\operatorname{ch}\left(\mathbb{Z}_{\ell}\left[\varpi_{\ell}\right]+\frac{j}{\varpi_{\ell}}\right), \varphi_{\ell, 2, j}=\operatorname{ch}\left(\left(\mathbb{Z}_{\ell}\left[\varpi_{\ell}\right]+\frac{j}{\varpi_{\ell}}\right)\left(\lambda^{-1} \mathcal{I}\right)\right)$ if $\ell>2$, and

$$
\left\{\begin{array}{l}
\varphi_{2,1,(i, j)}=\operatorname{ch}\left(\mathbb{Z}_{2}\left[\varpi_{2}\right]+\frac{1}{2}\left(i+\frac{j}{\varpi_{2}}\right)\right) \\
\varphi_{2,2,(i, j)}=\operatorname{ch}\left(\left(\mathbb{Z}_{2}\left[\varpi_{2}\right]+\frac{1}{2}\left(i+\frac{j}{\varpi_{2}}\right)\right)\left(\lambda^{-1} \mathcal{I}\right)\right) .
\end{array}\right.
$$

We now deal with $\varphi_{\ell}$ for primes $\ell \mid N$. Since these are split in $K=\mathbb{Q}(\sqrt{d})$ we can fix a $\delta_{\ell} \in \mathbb{Z}_{\ell}^{\times}$such that $\delta_{\ell}^{2}=d$ and if $\sqrt{d} \mapsto\left(\begin{array}{cc}a & b \\ c \ell^{v^{(}(N)} & -a\end{array}\right) \in \mathcal{R}\left(\ell^{v_{\ell}(N)}\right) \otimes \mathbb{Z}_{\ell}$ $(b c \neq 0)$ after localizing $\rho$ at $\ell$ and we may also assume that $\frac{1}{2}\left(\delta_{\ell}+a\right) \in \mathbb{Z}_{\ell}^{\times}$.

Lemma 3.8. Let $\ell \mid N$. With the notation just introduced, there exists $u_{\ell} \in$ $\rho(K)^{\perp} \otimes \mathbb{Q}_{\ell}$ such that $\varphi_{\ell}=\varphi_{\ell, 1} \otimes \varphi_{\ell, 2}$ where $\varphi_{\ell, 1}=\operatorname{ch}\left(\ell^{v_{\ell}(N)} \mathcal{O}_{K} \otimes \mathbb{Z}_{\ell}\right)$ and $\varphi_{\ell, 2}$ is the function supported on $\left(\ell^{-v_{\ell}(b)} \mathbb{Z}_{\ell}^{\times} \times \ell^{-v_{\ell}(c)} \mathbb{Z}_{\ell}\right) u_{\ell}$ such that $\varphi_{\ell, 2}\left((x, y) u_{\ell}\right)=$ $\varepsilon_{\ell}^{-1}\left(-x\left(a+\delta_{\ell}\right) b / 2\right)$.

Proof. The idempotents $e^{ \pm}=\frac{1}{2}\left(1 \otimes 1 \pm \sqrt{d} \otimes \delta_{\ell}^{-1}\right)$ define an isomorphism of rings $\mathcal{O}_{K} \otimes \mathbb{Z}_{\ell}=\mathbb{Z}_{\ell} e^{+} \times \mathbb{Z}_{\ell} e^{-} \simeq \mathbb{Z}_{\ell} \times \mathbb{Z}_{\ell}$ (this is true also if $\ell=2$ as $e^{ \pm}=1 \otimes \frac{\delta_{2} \pm 1}{2 \delta_{2}} \pm \frac{1+\sqrt{d}}{2} \otimes \delta_{2} \in \mathcal{O}_{K} \otimes \mathbb{Z}_{2}$ since $\left.\delta_{2} \equiv 1 \bmod 2\right)$. Obviously $\rho\left(e^{ \pm}\right)=$ $M^{ \pm}=\frac{1}{2 \delta_{\ell}}\left(\begin{array}{cc}\delta_{\ell} \pm a & \pm b \\ \pm c p^{v} \ell^{N} & \delta_{\ell} \mp a\end{array}\right)$. To get an explicit description of $\rho(K)^{\perp} \otimes \mathbb{Q}_{\ell}$ we can choose any $u_{\ell} \in \rho(K)^{\perp} \otimes \mathbb{Q}_{\ell}$ which generates it as a $\mathbb{Q}_{\ell} \times \mathbb{Q}_{\ell}$-module. Choose

$$
u_{\ell}=\left(1-e_{\rho}\right)\left(\begin{array}{cc}
1 & 0 \\
0 & -1
\end{array}\right)=\frac{1}{d}\left(\begin{array}{cc}
d-a^{2} & -a b \\
-a c \ell_{\ell}^{v_{\ell}(N)} & a^{2}-d
\end{array}\right) .
$$

Under our assumptions $\left(\ell^{v_{\ell}(b)}\right)^{-1} M^{+} u_{\ell}=\frac{1}{2 d \ell^{v_{\ell}(b)}}\left(\begin{array}{c}d-a^{2} \\ c\left(\delta_{\ell}-a\right) \ell^{v_{\ell}(N)}\end{array} \begin{array}{c}-b\left(a+\delta_{\ell}\right) \\ a^{2}-d\end{array}\right)$ and $\left(\ell^{v_{\ell}(c)}\right)^{-1} M^{-} u_{\ell}=\frac{1}{2 d \ell^{v_{\ell}(c)}}\left(\begin{array}{cc}d-a^{2} & b\left(\delta_{\ell}-a\right) \\ c\left(\delta_{\ell}+a\right) \ell^{v_{\ell}(N)} & a^{2}-d\end{array}\right)$ are a $\mathbb{Z}_{\ell}$-basis of $\left(\rho(K)^{\perp} \otimes \mathbb{Q}_{\ell}\right) \cap$ $\mathcal{R}_{\ell^{v_{\ell}(N), \ell}}$. It is now straightforward to check that given $\left(x^{+}, x^{-}, y^{+}, y^{-}\right) \in \mathbb{Z}_{\ell}^{4}$, $x^{+} M^{+}+x^{-} M^{-}+y^{+} \ell^{-v_{\ell}(b)} M^{+} u_{\ell}+y^{-} \ell^{-v_{\ell}(c)} M^{-} u_{\ell} \in S_{\ell}$ if and only if $x^{ \pm} \in$ $\ell^{v_{\ell}(N)} \mathbb{Z}_{\ell}, y^{+} \in \mathbb{Z}_{\ell}^{\times}$and $y^{-} \in \mathbb{Z}_{\ell}$. The final formula follows.

\subsection{Proof of main theorem 0.1}

Let $D$ be the quaternion algebra with discriminant $\Delta$. By hypothesis $\pi$ is the image of an automorphic representation $\pi^{D}$ of $D^{\times}$under the Jacquet-Langlands correspondence. Let $f^{\Delta} \in S_{2 k}^{\Delta}\left(N_{0}, \varepsilon\right)$ be a holomorphic newform in $\pi^{D}$ which we may assume to be defined over $\mathcal{O}_{(v)}$. For all $r \geq 0$ let $\phi_{f^{\Delta}}^{(r)}$ be as in (24) Following remark 3.6 write $\pi\left(d_{\infty}^{\tau}\right) \phi_{f^{\Delta}} \otimes \pi\left(d_{\infty}^{\tau}\right) \phi_{f^{\Delta}} \cdot\left(\varepsilon^{-1} \circ \nu\right)=v_{f^{\Delta}} \tilde{\theta}_{\varphi^{\sharp}}(F)$ where $F$ is the adelic lift of a normalized eigenform in $\pi, v=v_{f} \Delta \in \mathbb{C}^{\times}$a normalizing factor (a quotient of Petersson norms) and $\varphi^{\sharp}=\varphi_{\infty}^{\sharp} \otimes \varphi_{\text {fin }}^{\sharp}=\bigotimes_{v} \varphi_{v}^{\sharp}$.

Under the Lie algebra identifications $\mathfrak{g o}(D) \simeq\left(D_{\infty} \times D_{\infty}\right) / \mathbb{R}$ and $\mathfrak{o}(D) \simeq$ $\mathfrak{s l}_{2} \times \mathfrak{s l}_{2}$ induced by the exact sequence (26), the substitution formula (29) yields

$$
\tilde{\theta}_{H \varphi_{\infty} \otimes \varphi_{\mathrm{fin}}}(F)(h)=\left.\frac{d}{d t}\right|_{t=0} \tilde{\theta}_{\varphi_{\infty} \otimes \varphi_{\mathrm{fin}}}(F)(h \exp (t H))
$$


where $H \varphi_{\infty}(x)=\left.\frac{d}{d t}\right|_{t=0} \varphi_{\infty}\left(e^{-t H_{1}} x e^{t H_{2}}\right)$ for all $H=\left(H_{1}, H_{2}\right) \in \mathfrak{o}(D)$. In turn, this implies that the diagonal action of $A \in \mathfrak{s l}_{2}$ on $\pi^{D} \otimes \check{\pi}^{D}$ corresponds to the second order operator $A^{\prime}=(A, 0) \circ(0, A) \in \mathfrak{A}(\mathfrak{o}(D))$ on Schwartz functions, i.e. $A^{\prime} \varphi_{\infty}(x)=\left.\frac{\partial^{2}}{\partial u \partial v}\right|_{u=v=0} \varphi_{\infty}\left(e^{-u A} x e^{v A}\right)$. Note that since $\operatorname{tr}(A)=0$ this action leaves unchanged the factor $\varepsilon^{-1} \circ \nu$. Therefore

$$
\pi\left(d_{\infty}^{\tau}\right) \phi_{f^{\Delta}}^{(r)} \otimes \pi\left(d_{\infty}^{\tau}\right) \phi_{f^{\Delta}}^{(r)} \cdot\left(\varepsilon^{-1} \circ \nu\right)=v \tilde{\theta}_{\left(X_{\tau}^{\prime}\right)^{r} \varphi_{\infty}^{\sharp} \otimes \varphi_{\mathrm{fin}}^{\sharp}}(F) .
$$

where $X_{\tau}^{\prime}=\left(-\frac{1}{4 \pi} d_{\infty}^{\tau} X^{+}\left(d_{\infty}^{\tau}\right)^{-1}\right)^{\prime}$. Since

$$
\begin{aligned}
X_{\tau}^{\prime} \varphi_{\infty}^{\sharp}(x) & =\left.\frac{\partial^{2}}{\partial u \partial v}\right|_{u=v=0} \varphi_{\infty}^{\sharp}\left(e^{-u d_{\infty}^{\tau} X^{+}\left(d_{\infty}^{\tau}\right)^{-1}} x e^{v d_{\infty}^{\tau} X^{+}\left(d_{\infty}^{\tau}\right)^{-1}}\right) \\
& =\left.\frac{\partial^{2}}{\partial u \partial v}\right|_{u=v=0} \varphi_{\infty}^{\sharp}\left(d_{\infty}^{\tau} e^{-u X^{+}}\left(d_{\infty}^{\tau}\right)^{-1} x d_{\infty}^{\tau} e^{v X^{+}}\left(d_{\infty}^{\tau}\right)^{-1}\right) \\
& =\left(X^{+}\right)^{\prime} \varphi_{\infty}^{\prime}\left(\left(d_{\infty}^{\tau}\right)^{-1} x d_{\infty}^{\tau}\right)
\end{aligned}
$$

the computation of the operator $X_{\tau}^{\prime}$ reduces to that of $\left(X^{+}\right)^{\prime}$. Let $\mathfrak{h}=\mathbb{R}\left({ }_{1}{ }^{-1}\right)$ be the standard Cartan subalgebra of $\mathfrak{s l}_{2}$. The product $\mathfrak{h} \times \mathfrak{h}$ is identified to a Cartan subalgebra of $\mathfrak{o}(D)$ and if $e_{j} \in i \mathfrak{h}^{*}$ is the standard half-root of the $j$-th factor then we have the following table of roots and eigenvectors for the action of $\mathfrak{o}(D)$ on $D_{\infty}$ :

\begin{tabular}{|c|c|c|c|}
\hline$e_{1}+e_{2}$ & $e_{1}-e_{2}$ & $-e_{1}+e_{2}$ & $-e_{1}-e_{2}$ \\
\hline$E_{++}=\left(\begin{array}{cc}-i & 1 \\
1 & i\end{array}\right)$ & $E_{+-}=\left(\begin{array}{cc}-i & -1 \\
1 & -i\end{array}\right)$ & $E_{-+}=\left(\begin{array}{cc}i & -1 \\
1 & i\end{array}\right)$ & $E_{--}=\left(\begin{array}{cc}i & 1 \\
1 & -i\end{array}\right)$ \\
\hline
\end{tabular}

The action of the operators $\left(X^{+}, 0\right)$ and $\left(0, X^{+}\right)$on these eigenvectors is

$$
\begin{array}{ll}
\left(X^{+}, 0\right) E_{+ \pm}=0 & \left(X^{+}, 0\right) E_{- \pm}=-E_{+ \pm} \\
\left(0, X^{+}\right) E_{ \pm,+}=0 & \left(0, X^{+}\right) E_{ \pm-}=-E_{ \pm+}
\end{array}
$$

Comparing the expressions of $x \in D_{\infty}$ in terms of the complex standard coordinates $z_{i}=z_{i}^{\text {st }}$ and in the real coordinates with respect to the above eigenvectors allows to compute the expression of $\left(X^{+}, 0\right)$ and $\left(0, X^{+}\right)$in terms of the standard coordinates. Eventually

$$
\left(X^{+}\right)^{\prime}=z_{2}^{2} \frac{\partial^{2}}{\partial z_{1} \partial \bar{z}_{1}}+\bar{z}_{1} z_{2} \frac{\partial^{2}}{\partial \bar{z}_{1} \partial \bar{z}_{2}}+z_{1} z_{2} \frac{\partial^{2}}{\partial z_{1} \partial \bar{z}_{2}}+z_{1} \bar{z}_{1} \frac{\partial^{2}}{\partial \bar{z}_{2}{ }^{2}}+z_{2} \frac{\partial}{\partial \bar{z}_{2}} .
$$

For a pair $(l, m)$ of non-negative integers, consider the function of two complex variables $\varphi^{(l, m)}\left(z_{1}, z_{2}\right)=\left(z_{1} \bar{z}_{1}\right)^{l} z_{2}^{2 m} e^{-2 \pi\left(z_{1} \bar{z}_{1}+z_{2} \bar{z}_{2}\right)}$. Then

$$
\left(X^{+}\right)^{\prime} \varphi^{(l, m)}=l^{2} \varphi^{(l-1, m+1)}-4 \pi(2 l+1) \varphi^{(l, m+1)}+(4 \pi)^{2} \varphi^{(l+1, m+1)} .
$$

and a $r$-fold iteration allows to conclude eventually that

$$
\begin{aligned}
\pi\left(d_{\infty}^{\tau}\right) \phi_{f}^{(r)} \otimes \pi\left(d_{\infty}^{\tau}\right) \phi_{f}^{(r)}\left(\varepsilon^{-1} \circ \nu\right)= & \\
v & \left(\tilde{\theta}_{\varphi^{\sharp(r, r)} \otimes \varphi_{\mathrm{fin}}^{\sharp}}(F)+\sum_{l=0}^{r-1} a_{r, l} \tilde{\theta}_{\varphi^{\sharp(l, r)} \otimes \varphi_{\mathrm{fin}}^{\sharp}}(F)\right)
\end{aligned}
$$


for some $a_{r, l} \in \mathbb{Z}[1 / 4 \pi]$ and $\varphi^{\sharp(l, r)}(x)=\frac{1}{\pi} \varphi^{(l, \kappa+r)}\left(\left(d_{\infty}^{\tau}\right)^{-1} x d_{\infty}^{\tau}\right)$. The function $\varphi^{\sharp, l}$ is split under the decomposition of $D_{\infty} \ni x=\rho\left(z_{1}\right)+\rho\left(z_{2}\right) u$ induced by $\rho$, namely $\varphi^{\sharp(l, r)}=\frac{1}{\pi} \varphi_{1}^{\sharp l} \otimes \varphi_{2}^{\sharp, r}$ with

$$
\varphi_{1}^{\sharp, l}(x)=\left(z_{1} \bar{z}_{1}\right)^{l} e^{-2 \pi i z_{1} \bar{z}_{1}}, \quad \varphi_{2}^{\sharp, r}(x)=\zeta_{u}^{2(\kappa+r)} z_{2}^{2(\kappa+r)} e^{-2 \pi i|\nu(u)| z_{2} \bar{z}_{2}} .
$$

\section{Lemma 3.9.}

$$
\begin{aligned}
& L_{\infty}\left(\varphi^{\sharp(l, r)}, \xi_{r, \infty}, s\right)= \\
& \quad= \begin{cases}2 \frac{\overline{j(u, \tau)}}{j(u, \tau)^{\kappa+r+\frac{1}{2}}} \frac{r !}{(4 \pi)^{s+2(\kappa+r)+\frac{1}{2}}} \Gamma\left(s+2 \kappa+r+\frac{1}{2}\right) & \text { if } l=r, \\
0 & \text { if } 0 \leq l<r .\end{cases}
\end{aligned}
$$

Proof. We shall compute separately the three main ingredients of the integrand in the local $L_{\infty}$ factor and piece them together later.

It is well known that $W_{F}^{\psi_{\infty}}\left(\left(\begin{array}{c}a \\ \end{array}\right) r_{\theta}\right)=\operatorname{ch}_{\mathbb{R}^{\times}}(a) a^{\kappa} e^{-2 \pi a-2 \kappa i \theta}$.

To compute $\Phi_{\varphi_{1}^{\sharp, l}}^{s}\left(\left(\begin{array}{c}a \\ { }_{1}\end{array}\right) r_{\theta}\right)=|a|^{s} r_{\psi_{\infty}}\left(r_{\theta}\right) \varphi_{1}^{\sharp, l}(0)$ we use the decomposition

$$
\begin{aligned}
r_{\theta}=\left(\begin{array}{cc}
1 & -\tan \theta \\
0 & 1
\end{array}\right)\left(\begin{array}{cc}
0 & 1 \\
-1 & 0
\end{array}\right)\left(\begin{array}{cc}
1 & -\sin \theta \cos \theta \\
0 & 1
\end{array}\right) . \\
\\
\left(\begin{array}{cc}
0 & 1 \\
-1 & 0
\end{array}\right)\left(\begin{array}{cc}
1 / \cos \theta & 0 \\
0 & \cos \theta
\end{array}\right) .
\end{aligned}
$$

Applying repeatedly we get $r_{\psi_{\infty}}\left(r_{\theta}\right) \varphi_{1}^{\sharp l}(0)=-g(0) \cos \theta$ where $g(z)$ is the Fourier transform of $\hat{\varphi}_{1}^{\sharp, l}(z \cos \theta) e^{-2 \pi i \cos \theta \sin \theta|z|^{2}}$. The Fourier transform $\hat{\varphi}_{1}^{\sharp l}$ is given by

$$
\hat{\varphi}_{1}^{\sharp, l}\left(x_{1}+x_{2} i\right)=e^{-2 \pi\left(x_{1}^{2}+x_{2}^{2}\right)} \sum_{0 \leq \alpha+\beta \leq l} \gamma_{\alpha, \beta}^{l} x_{1}^{2 \alpha} x_{2}^{2 \beta}
$$

with coefficients $\gamma_{\alpha, \beta}^{l}=\sum(-4 \pi)^{\alpha+\beta-l}\left(\begin{array}{l}l \\ j\end{array}\right)\left(\begin{array}{l}2 j \\ 2 \alpha\end{array}\right)\left(\begin{array}{l}2 k \\ 2 \beta\end{array}\right)(2 j-2 \alpha-1) ! !(2 k-2 \beta-1) ! !$. Therefore

$$
\begin{aligned}
g(0) & =\int_{\mathbb{C}} \hat{\varphi}_{1}^{\sharp l}(z \cos \theta) e^{-2 \pi i \cos \theta \sin \theta|z|^{2}} d z \\
= & 2 \sum_{0 \leq \alpha+\beta \leq l} \gamma_{\alpha, \beta}^{l}(\cos \theta)^{2(\alpha+\beta)} \int_{\mathbb{R}^{2}} e^{-2 \pi\left(i \sin \theta \cos \theta+(\cos \theta)^{2}\right)\left(x^{2}+y^{2}\right)} x^{2 \alpha} y^{2 \beta} d x d y \\
& =-\sum_{0 \leq \alpha+\beta \leq l} \gamma_{\alpha, \beta}^{l} \frac{(2 \alpha-1) ! !(2 \beta-1) ! !}{(4 \pi)^{\alpha+\beta}} \frac{(\cos \theta)^{2(\alpha+\beta)}}{\left(-\sin \theta \cos \theta-(\cos \theta)^{2}\right)^{\alpha+\beta+1}}
\end{aligned}
$$

and eventually

$$
\begin{aligned}
\Phi_{\varphi_{1}^{\sharp, l}}^{s}\left(\left(\begin{array}{ll}
a & 0 \\
0 & 1
\end{array}\right) r_{\theta}\right) & = \\
& |a|^{s} \sum_{0 \leq \alpha+\beta \leq l} \frac{\gamma_{\alpha, \beta}^{l}(2 \alpha-1) ! !(2 \beta-1) ! !}{(4 \pi)^{\alpha+\beta}}(\cos \theta)^{\alpha+\beta} e^{-(\alpha+\beta+1) i \theta} .
\end{aligned}
$$


Also the computation of $W_{\theta_{\varphi_{2}^{\sharp}, \infty}}^{\psi_{\infty}}$ uses (38) and goes as in [27]: we summarize it briefly for the sake of completeness. Note first that the norm in $(K u) \otimes \mathbb{R} \simeq \mathbb{C}$ is $-\mathrm{N}_{\mathbb{C} / \mathbb{R}}$ (definite negative) and the main involution is $z \mapsto-z$.

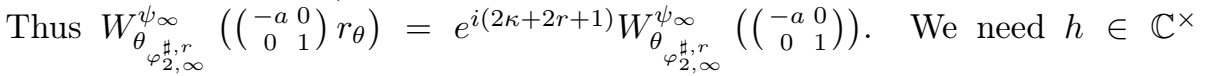
such that $|h|=-a \nu(u)^{-1}$ : such $h$ exists only if $a>0$ and so we may pick $h=\sqrt{a|\nu(u)|^{-1}} \in \mathbb{R}^{\times}$. We have

$$
\begin{aligned}
r_{\psi}\left(\left(\begin{array}{cc}
a|\nu(u)|^{-1} & 0 \\
0 & 1
\end{array}\right), h \vartheta\right) \varphi_{2}^{\sharp, l}(u) & =\left(a|\nu(u)|^{-1}\right)^{\frac{1}{2}} \varphi_{2}^{\sharp, l}\left(a|\nu(u)|^{-1}(h \vartheta)^{-1} u\right) \\
& =\left(a|\nu(u)|^{-1}\right)^{\frac{1}{2}}\left(\zeta_{u} h \vartheta^{-1}\right)^{2(\kappa+l)} e^{-2 \pi a}
\end{aligned}
$$

so that

$$
\begin{aligned}
& W_{\theta_{\varphi_{2}^{\sharp}, \infty}^{\sharp}}^{\psi_{\infty}}\left(\begin{array}{cc}
-a & 0 \\
0 & 1
\end{array}\right)=\int_{S^{1}} r_{\psi}\left(\left(\begin{array}{cc}
a|\nu(u)|^{-1} & 0 \\
0 & 1
\end{array}\right), h \vartheta\right) \varphi_{2}^{\sharp, r}(u) \xi_{r . \infty}(h \vartheta) d \vartheta \\
& =\zeta_{u}^{2(\kappa+l)} \operatorname{ch}_{\mathbb{R}>0}(a)|\nu(u)|^{-\frac{2(\kappa+r)+1}{2}} a^{\frac{2(\kappa+r)+1}{2}} e^{-2 \pi a} \text {. }
\end{aligned}
$$

Summing up:

$$
\begin{gathered}
L_{\infty}\left(\varphi^{\sharp(l, r)}, \xi_{r, \infty}, s\right)=\frac{1}{\pi} \zeta_{u}^{2(\kappa+r)}|\nu(u)|^{\frac{-2(\kappa+r)-1}{2}} \\
\times \int_{S^{1}} \int_{0}^{\infty} a^{2 \kappa+r+s-\frac{1}{2}} e^{-4 \pi a} \sum_{0 \leq \alpha+\beta \leq l} \delta_{\alpha, \beta}^{l}(\cos \vartheta)^{\alpha+\beta} e^{(2 r-\alpha-\beta) i \vartheta} d a d \vartheta= \\
\frac{\xi_{\infty, r}\left(\zeta_{u}\right)}{|\nu(u)|^{\frac{1}{2}}} \frac{\Gamma\left(2 \kappa+r+s+\frac{1}{2}\right)}{\pi(4 \pi)^{2 \kappa+r+s+\frac{1}{2}}} \sum_{0 \leq \alpha+\beta \leq l} \frac{\delta_{\alpha, \beta}^{l}}{2^{\alpha+\beta}} \sum_{j=0}^{\alpha+\beta}\left(\begin{array}{c}
\alpha+\beta \\
j
\end{array}\right) \int_{S^{1}} e^{2(r+j-\alpha-\beta) i \vartheta} d \vartheta
\end{gathered}
$$

where $\delta_{\alpha, \beta}^{l}=\gamma_{\alpha, \beta}^{l}(2 \alpha-1) ! !(2 \beta-1) ! !(4 \pi)^{-\alpha-\beta}$. Since the last integral is 0 unless $\alpha+\beta=l=r$ and $j=0$, this proves the claim for $0 \leq l<r$.

When $\alpha+\beta=l=r$ a straightforward induction shows that $\sum_{\alpha=0}^{r} \delta_{\alpha, r-\alpha}^{r}=$ $(4 \pi)^{-r} \sum_{\alpha=0}^{r}\left(\begin{array}{l}r \\ \alpha\end{array}\right)(2 \alpha-1) ! !(2 r-2 \alpha-1) ! !=(4 \pi)^{-r} 2^{r} r !$ so that

$$
L_{\infty}\left(\varphi^{\sharp(r, r)}, \xi_{r, \infty}, s\right)=2 \frac{\xi_{\infty, r}\left(\zeta_{u}\right)}{|\nu(u)|^{\frac{1}{2}}} \frac{r !}{(4 \pi)^{2(\kappa+r)+s+\frac{1}{2}}} \Gamma\left(2 \kappa+r+s+\frac{1}{2}\right) .
$$

Applying the cochain relation for the automorphy factor $j(\gamma, \tau)$ to both sides of the identity $\left(d_{\infty}^{\tau}\right)^{-1} u d_{\infty}^{\tau}=\zeta_{u}\left(\begin{array}{ll}{ }^{1} & \\ & \end{array}\right)$ and observing that $u \cdot \tau=\bar{\tau}$ one eventually obtains $\zeta_{u}=-\overline{j(u, \tau)}$ and the formula follows.

Since the archimedean component $\left(\pi_{K}\right)_{\infty}$ is the representation $\pi(\lambda, \lambda)$ of $\mathrm{GL}_{2}(\mathbb{C})$ associated to the character $\lambda(z)=(z \bar{z})^{-\kappa+\frac{1}{2}} z^{2 \kappa-1}$ we can write

$$
\left.L_{\infty}\left(\varphi^{\sharp(r, r)}, \xi_{r, \infty}, s\right)\right|_{s=\frac{1}{2}}=\left.\Lambda_{\infty}\left(\varphi^{\sharp(r, r)}, \xi_{r, \infty}, s\right) L_{\infty}\left(\pi_{K} \otimes \xi_{r}, s\right)\right|_{s=\frac{1}{2}}
$$




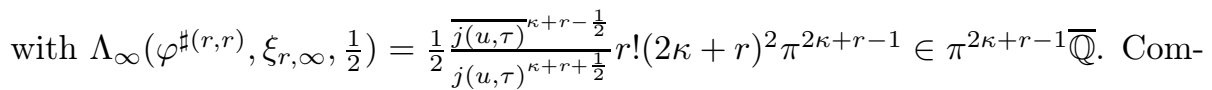
bining the lemma with (32) and (35) yields

$$
\begin{aligned}
J_{r}\left(f^{\Delta}, \xi_{r}, \tau\right)^{2}= & L_{\xi_{r}}\left(\tilde{\theta}_{\varphi^{\sharp(r, r)}} \otimes \varphi_{\mathrm{fin}}(F)\right)= \\
& v \pi^{2 \kappa+r-1} \Lambda\left(\varphi^{\sharp(r, r)} \otimes \varphi_{\mathrm{fin}}, \xi_{r}, \frac{1}{2}\right) L\left(\pi_{K} \otimes \xi_{r}, \frac{1}{2}\right) L\left(\eta_{K}, 1\right)^{-1}
\end{aligned}
$$

where the archimedean factor $\Lambda_{\infty}$ has been redefined so to make explicit the correct power of $\pi$.

On the other hand, assuming $\mathcal{O}_{K, c}^{\times}=\{ \pm 1\}$, from proposition 3.2

$$
J_{r}\left(f^{\Delta}, \xi_{r}, \tau\right)^{2}=\pi^{2} \operatorname{vol}\left(\hat{\mathcal{O}}_{K, c}^{\times}\right)^{2}\left(h_{c}^{\sharp}\right)^{2}\left(y \Omega_{\infty}(\tau)^{2}\right)^{2(\kappa+r)}\left\langle\vartheta\left(r, f^{\Delta}, x\right), \chi_{r}\right\rangle_{\infty}^{2} .
$$

When $\chi_{r}$ is the $r$-th moment of a $\mathfrak{F}\left(c_{\varepsilon}^{*}, F\right)$-valued measure on $\mathbb{Z}_{p}$ propositions 2.12 and 2.16 apply to define a measure $\mu$ on $\mathbb{Z}_{p}$ such that

$$
\frac{1}{\Omega_{p}^{4(\kappa+r)}}\left(\int_{\mathbb{Z}_{p}} t^{r} d \mu(t)\right)^{2}=\left(\frac{1}{\pi \operatorname{vol}\left(\hat{\mathcal{O}}_{K, c}^{\times}\right) h_{c}^{\sharp}\left(y \Omega_{\infty}(\tau)^{2}\right)^{\kappa+r}}\right)^{2} J_{r}\left(f^{\Delta}, \xi_{r}, \tau\right)^{2} .
$$

The last part of proposition 2.12 and corollary 2.17 yield

$$
\begin{aligned}
\frac{1}{\Omega_{p}^{4(\kappa+r)}}\left(\int_{\mathbb{Z}_{p}^{\times}} t^{r} d \mu(t)\right)^{2}= \\
\\
\quad\left(\frac{1}{\pi \operatorname{vol}\left(\hat{\mathcal{O}}_{K, c}^{\times}\right) h_{c}^{\sharp}\left(y \Omega_{\infty}(\tau)^{2}\right)^{\kappa+r}}\right)^{2} J_{r}\left((1-V U) f^{\Delta}, \xi_{r}, \tau\right)^{2} .
\end{aligned}
$$

Then one concludes applying corollary 3.4, plugging (40) in and taking into account remark 2.14 .

\section{References}

[1] M. Bertolini, H. Darmon and K. Prasanna. Generalized Heegner cycles and p-adic Rankin L-series. With an appendix by Brian Conrad. Duke Math J., 162:1033-1148, 2013.

[2] M. Brakočević. Anticyclotomic p-adic L-function of central critical RankinSelberg L-value, Int. Math. Res. Not. IMRN, 21:4967-5018, 2011.

[3] D. Bump. Automorphic Forms and Representations, volume 55 of Cambridge Studies in Adv. Math.. Cambridge University Press, 1996.

[4] K. Buzzard. Integral models of certain Shimura curves, Duke Math. J., 87:591-611, 1997. 
[5] P. Clark. Rational points on Atkin-Lehner quotients of Shimura curves, Ph.D. thesis, Harvard University, 2003.

[6] G. Faltings and C.-L. Chai. Degenerations of Abelian Varieties, volume 22 of Ergebnisse der Math. Springer, 1990.

[7] F. Gouvêa Arithmetic of p-adic modular forms, volume 1304 of Lecture Notes in Math.

[8] M. Harris. Special values of zeta functions attached to Siegel modular forms. Ann. Sc. Éc. Norm. Sup., 14:77-120, 1981.

[9] M. Harris. Non-vanishing of $L$-functions on $2 \times 2$ unitary groups. Forum Math., 5:405-419, 1993.

[10] M. Harris and S. Kudla. The central critical value of a triple product L-function. Annals Math., 133:605-672, 1991.

[11] M. Harris and S. Kudla. Arithmetic automorphic forms for the nonholomorphic discrete series of GSp(2). Duke Math. J., 66:59-121, 1992.

[12] M. Harris and J. Tilouine. p-adic measures and square roots of special values of triple product $L$-functions. Math. Ann., 320:127-147, 2001.

[13] K. Hashimoto. Explicit form of quaternion modular embeddings. Osaka J. Math., 32:533-546, 1995.

[14] H. Hida. Hecke algebras for $\mathrm{GL}_{1}$ and $\mathrm{GL}_{2}$. In Seminaire de theorie des nombres, Paris 1983-84, volume 63 of Progr. Math., pages 133-146, 1986.

[15] H. Jacquet. Automorphic forms on GL(2), II, volume 278 of Lecture Notes in Math. Springer-Verlag, 1972.

[16] H. Jacquet and R. P. Langlands. Automorphic forms on GL(2), volume 114 of Lecture Notes in Math. Springer-Verlag, 1970.

[17] P. Kassaei. $p$-adic modular forms over Shimura curves over $\mathbb{Q}$. Ph.D. thesis, M.I.T. 1999.

[18] N. Katz. p-adic properties of modular schemes and modular forms. In Modular Functions of One Variable III, volume 350 of Lecture Notes in Math., pages 70-189, 1973.

[19] N. Katz. p-adic interpolation of real analytic Eisenstein series. Annals of Math., 104:459-571, 1976.

[20] N. Katz. p-adic L-function for CM fields. Inventiones Math., 49:199-297, 1978.

[21] N. Katz. Serre-Tate local moduli. In Surfaces Algebraiques, volume 868 of Lecture Notes in Math., pages 138-202, 1981. 
[22] N. Katz and B. Mazur. Arithmetic moduli of elliptic curves, volume 108 of Annals of Math. Studies. Princeton Univ. Press, 1985.

[23] N. Katz and T. Oda. On the differentiation of de Rham cohomology classes with respect to parameters. J. Math. Kyoto Univ., 8(2):199-213, 1968.

[24] S. Kudla. Seesaw dual reductive pairs. In Automorphic forms of several variables (Katata 1983), volume 46 of Progr. Math, pages 244-268. Birkhäuser Boston, 1984.

[25] A. Mori. A characterization of integral elliptic automorphic forms. Ann. Sc. Norm. Sup. Pisa, IV(21):45-62, 1994.

[26] A. Mori. Power series expansions of modular forms and their interpolation properties. Int. J. Number Theory, 7(2):529-577, 2011.

[27] K. Prasanna. Integrality of a ratio of Petersson norms and level-lowering congruences. Ann. Math., 163:901-967, 2006.

[28] H. Shimizu. Theta series and automorphic forms on $\mathrm{GL}_{2}$. Math. Soc. Japan, 24:638-683, 1972.

[29] G. Shimura. Introduction to the Arithmetic Theory of Automorphic Functions. Iwanami Shoten and Princeton University Press, 1971.

[30] J.-L. Waldspurger. Sur les valeurs des certaines fonctions $L$ automorphes en leur centre de symétrie. Compositio Math., 54:173-242, 1985.

[31] T. Watson. Rankin triple products and quantum chaos. Ph.D. thesis, Princeton University, 2003.

[32] A. Weil. On a certain type of characters of the idele-class group of an algebraic number-field. In Proceedings of the international symposium on algebraic number theory, Tokio \&3 Nikko 1955, pages 1-7, 1956. 